\title{
CLL cells cumulate genetic aberrations prior to the first therapy even in outwardly inactive disease phase
}

\author{
María Hernández-Sánchez ${ }^{1,2}$ • Jana Kotaskova ${ }^{3,4} \cdot$ Ana E Rodríguez $^{1,2}$ • Lenka Radova ${ }^{3}$ - David Tamborero ${ }^{5,6}$. \\ María Abáigar $^{1,2} \cdot$ Karla Plevova $^{3,4} \cdot$ Rocío Benito $^{1,2} \cdot$ Nikola Tom $^{3} \cdot$ Miguel Quijada-Álamo $^{1,2} \cdot$ Vasileos Bikos $^{3}$.

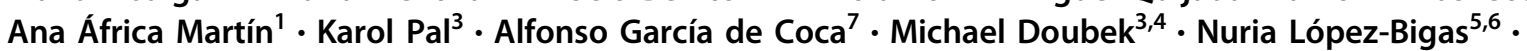 \\ Jesús-María Hernández-Rivas ${ }^{1,2} \cdot$ Sarka Pospisilova ${ }^{3,4}$
}

Received: 16 May 2018 / Revised: 3 August 2018 / Accepted: 9 August 2018 / Published online: 9 December 2018

(c) The Author(s) 2018. This article is published with open access

Over the past few years, several large-scale studies using next-generation sequencing (NGS) of whole-genomes (WGS) and whole-exomes (WES) have defined the mutational landscape of chronic lymphocytic leukemia (CLL) [1-4]. NGS studies have also revealed the clonal

These authors contributed equally: María Hernández-Sánchez, Jana Kotaskova

These authors jointly supervised this work: Jesús-María HernándezRivas, Sarka Pospisilova

Electronic supplementary material The online version of this article (https://doi.org/10.1038/s41375-018-0255-1) contains supplementary material, which is available to authorized users.

Jesús-María Hernández-Rivas

jmhr@usal.es

$\triangle$ Sarka Pospisilova

pospisilova.sarka@fnbrno.cz

1 Hematology Department, Hospital Universitario Salamanca, Salamanca, Spain

2 IBSAL, IBMCC-Cancer Research Center, University of Salamanca, Salamanca, Spain

3 Center of Molecular Medicine, Central European Institute of Technology, Masaryk University, Brno, Czech Republic

4 Center of Molecular Biology and Gene Therapy, Department of Internal Medicine - Hematology and Oncology, University Hospital Brno and Medical Faculty, Masaryk University, Brno, Czech Republic

5 Research Programon Biomedical Informatics, IMIM Hospital del Mar Medical Research Institute and Universitat Pompeu Fabra, Barcelona, Spain

6 Catalan Institution for Research and Advanced Studies (ICREA), Barcelona, Spain

7 Hematology Department, Hospital Clínico Universitario of Valladolid, Valladolid, Spain heterogeneity in CLL and showed that clonal evolution contributes to the variability in clinical course among CLL patients [3]. Clonal evolution is considered a key condition in CLL progression and relapse after treatment. Most CLL cases are diagnosed during the inactive disease phase, genetic aberrations' underlying progress in CLL activity leading to the need for therapy are poorly understood and should be explored. A large number of frequently mutated genes have been identified and several putative driver mutations likely to confer selective growth advantage to CLL tumor cells have been proposed [1-3]. In addition, clonal shifts between paired treatment-naïve and relapsed CLL samples have been reported due to pre-existing subclone expansion under therapeutic pressure, demonstrating that clonal evolution likely underlies CLL relapse $[3,5]$. Nevertheless, there are still a limited amount of longitudinal WES studies analyzing consecutive CLL samples before treatment intervegntion [6]. The acquisition of driver mutations accompanied by selectively neutral passenger changes during disease prior to therapy influence is therefore poorly documented. Here, WES was performed on consecutive treatment-naïve samples of CLL patients from three groups with different disease course: Active disease (AD) group: patients with an active disease before the second analyzed time-point (TP2); Stable disease (SD) group: cases with a period of stable phase after diagnosis followed by progression within 3 years after; and Indolent disease (ID) group: those with a long-term stable indolent disease. Moreover, we applied a novel integrative bioinformatics tool called "Cancer Genome Interpreter" to identify driver mutations [7].

Thirty-five CLL patients were included in the WES study. In total, 70 tumor samples - (two tumor time-points (TP) for each patient) - as well as 26 matched germline samples, were sequenced. Three groups of patients were characterized based on the disease activity at the second TP: 


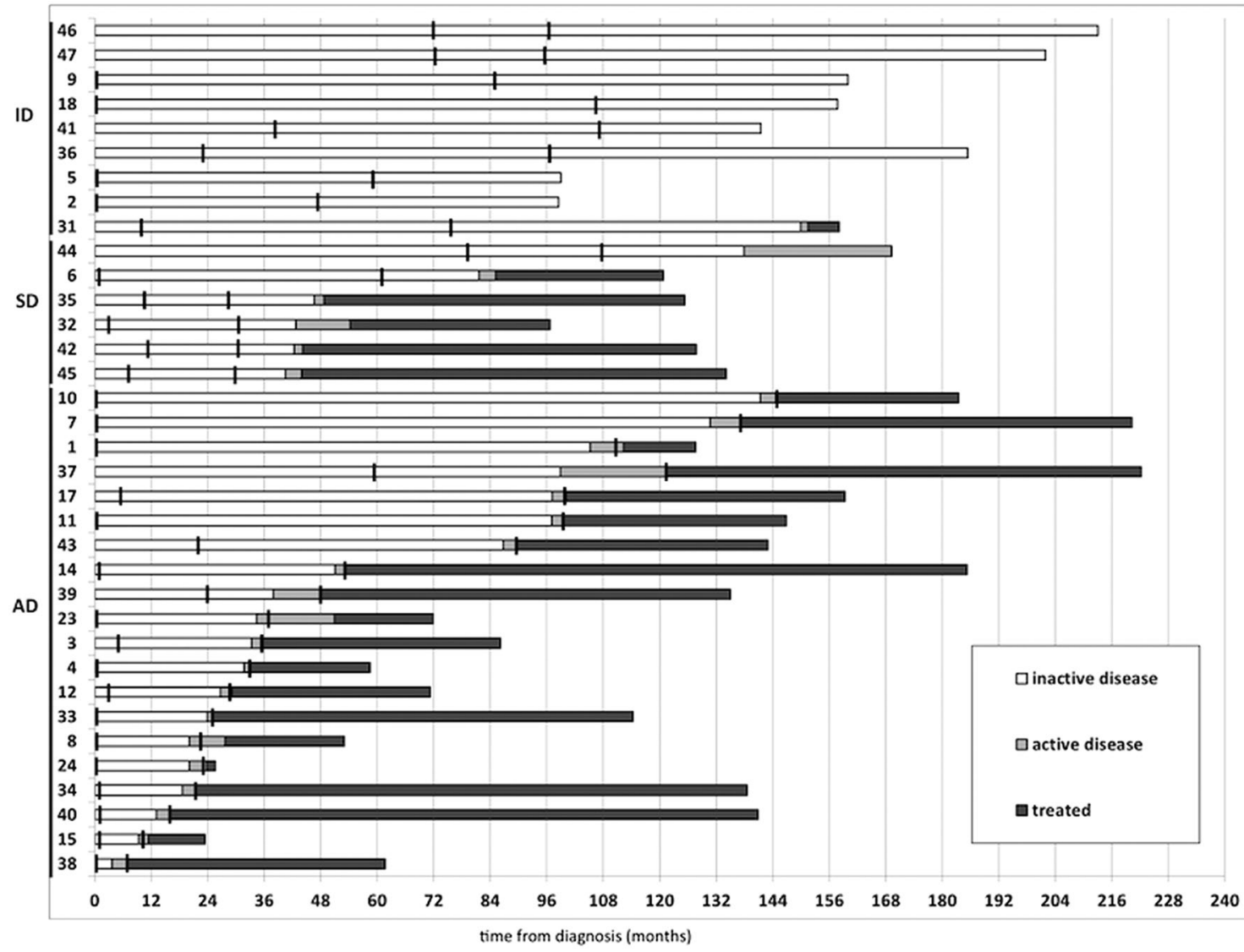

Fig. 1 Sampling points and follow-ups of the tested cohort. Time-point 1 (TP1) for all tested samples was set to the inactive stage of the disease close to the diagnosis (median from diagnosis to TP1 $=$ 2.1 months). Second time-points (TP2) were all collected prior to CLL-related therapy. Three groups of patients were then characterized based on disease activity in the TP2: (i) Active disease (AD) group patients with TP2 in disease's active phase $(n=20$, median TTP $=$ 33.9 months, median TTFT $=41.9$ months); (ii) Stable disease (SD)

(i) AD group $(n=20)$; (ii) SD group $(n=6)$; (iii) ID group $(n=9)$. Sampling points and group definition details are shown in Fig. 1. The disease activity was assessed according to iwCLL guidelines [8]. Sample characteristics are summarized in Supplementary Table S1, sample processing and WES analysis are detailed in Supplemental Material. In order to distinguish driver from passenger mutations, the novel bioinformatics tool "Cancer Genome Interpreter" (CGI, https://www.cancergenomeinterpreter. org/home) was used; [7] defined driver mutations were consequently validated by deep-targeted sequencing (DTS), as described previously [9]. Moreover, FISH data from testing of four recurrent cytogenetic aberrations (del13q, trisomy 12, del11q and del17p) were available for all samples.

WES analysis of samples from both TPs obtained from 26 CLL patients with available paired germline material showed presence of 25 somatic mutations. From WES analysis of 9 CLL patients with no available non-tumor group - patients with TP2 in disease's inactive phase followed by active phase and therapy need $(n=6$, median of time to progression $(\mathrm{TTP})=44.7$ months, median of time to first treatment $($ TTFT $)=$ 46.8 months); (iii) Indolent disease (ID) group - patients with TP2 taken in inactive phase, no disease activity or therapy need was reached during follow-up of 3 years $(n=9$, median follow-up $=$ 158.1 months). Only one ID case (P31) progressed after 150 months and required therapy intervention

control, 67 putatively somatic mutations were identified. Taken together, a total of 392 non-silent somatic or putatively somatic mutations (363 non-synonymous and 29 indels) were identified in 353 genes across the 35 CLL patients (Supplementary Table S2). Using CGI algorithm, 54 mutations were classified as "driver" and 338 mutations as "passenger" (Supplementary Table S2). The large majority of driver mutations $(50 / 54,92.6 \%)$ were further validated by deep-targeted sequencing (DTS) (Supplementary Table S3). Moreover, DTS of a 9-gene set recurrently mutated in CLL (TP53, SF3B1, NOTCH1, NFKBIE, BIRC3, POT1, MYD88, XPO1, and EGR2) revealed 7 mutations which were not detected by WES due to their low Variant Allele Frequency (VAF) (Supplementary Table S3). The 57 validated driver mutations were located in 35 different genes. The most frequently mutated genes were SF3B1 (8/35, 22.9\%), NOTCH1 (4/35, 11.4\%), NFKBIE (4/ $35,11.4 \%), \operatorname{TP} 53(3 / 35,8.6 \%), B I R C 3(3 / 35,8.6 \%)$, and $\operatorname{RPS} 15$ (3/35, 8.6\%) (Fig. 2). Among the other genes with a 


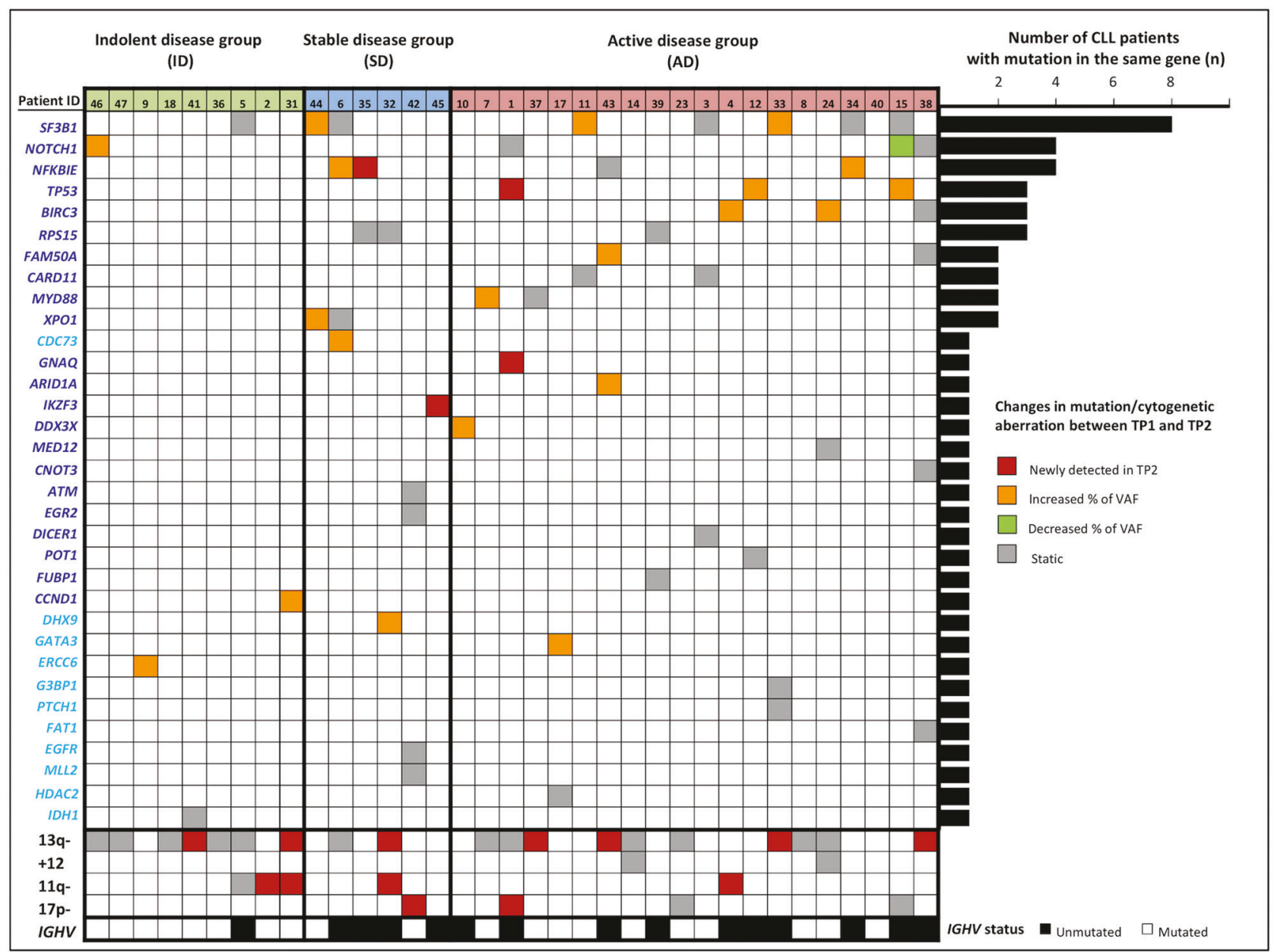

Fig. 2 List of genes with driver mutations validated using deep targeted sequencing and their changes in variant allele frequency between time-points. Cut-off 5\% of VAF was applied for validation of identified mutations. Allele frequency differences were tested across leukemia samples using a Fisher's exact test. Mutations were considered to be changed if they were significantly different between samples

driver mutation, 11 had previously been reported as drivers in CLL patients [2,3]. Additionally, CGI analysis also predicted driver mutations in $C D C 73, D H X 9, E G F R$, ERCC6, FAT1, GATA3, G3BP1, HDAC2, IDH1, and PTCH1 genes that were unknown for CLL to date (Fig. 2). Among them, the tumor suppressor $F A T 1$ has been related to chemo-refractoriness in CLL [10]; HDAC2 is known to be down-regulated in CLL [11]; and DHX9, GATA3, and IDHI have been described to be recurrently mutated in other hematological malignancies [12].

To identify somatic mutations which could be involved in clonal evolution, we analyzed the VAF dynamics between TP1 and TP2. Twenty-six out of 57 (46\%) driver mutations showed a significant change in allele frequency at the TP2: 4 were detected only at the TP2, 21 showed VAF increase at the TP2, and 1 mutation showed a decrease (Fig. 2). Additionally, FISH analysis of four recurrent (evolution $p$-value $<0.05$ ) and their ratio of VAF between TP2 and TP1 was higher than 1.5 (for "increased" mutations) or lower than 0.375 (for "decreased" mutations). Genes with dark blue labels (on the left axis) were CLL drivers previously identified in Puente et al. ${ }^{2}$ and Landau et al. ${ }^{3}$ and, where those in light blue were unknown as CLL drivers to date

cytogenetic aberrations at both TPs showed that $11 / 35$ patients acquired one or more new cytogenetic alterations at TP2 (3/9 ID, 2/6SD, and 6/20 AD) (Fig. 2). The most often acquired aberration - deletion 13q, was detected in 7 cases (2/9 ID, 1/6SD, and 4/20 AD). Acquisition of deletion 11q was detected in 4 cases (2/9 ID, 1/6SD, and 1/20 AD). Two patients who acquired a $17 \mathrm{p}$ deletion were from the SD and AD group. Taking together the WES and FISH results, clonal evolution was observed in 5/9 ID patients, in 6/6SD patients and in 14/20 AD patients. Of note, 5/9 ID patients showed clonal evolution although they showed a long-term indolent disease (median follow-up $=158$ months). Mutations in CLL drivers associated with aggressive clinical course such as TP53, BIRC3, RPS15, and NFKBIE [4, 13-15] were mostly detected within the AD/SD groups (Fig. 2). Nevertheless, there were well-known CLL driver mutations (NOTCH1, SF3B1) detected in two of eight ID patients, 
revealing the fact that the simple presence of such a mutation does not immediately lead to disease progression. Follow-up of these two patients with indolent disease already bearing a driver mutation at TP1 reached 99 (P5), and 213.1 (P46) months with no clinical evidence of disease activity to date as documented in Supplementary Table 1 (Fig. 1).

In summary, we performed a longitudinal study using whole-exome sequencing to characterize genetic alterations occurring during disease course before CLL-related therapy intervention in 35 CLL patients. We compared samples from indolent CLL to samples from a stable or active disease. To define potential driver mutations, we used novel integrative bioinformatics tool "Cancer Genome Interpreter". We showed continual evolution with cytogenetic aberration and somatic mutation accumulation during the time prior to therapy intervention. Despite clonal evolution, including driver mutation presence in genes such as NOTCH1 or SF3B1, observed in indolent CLL cases, there was no clinical evidence of disease activity during longterm follow-up after sampling. We conclude that the acquisition of aberrations is not limited to the active disease phase or relapses after therapy $[3,5,6]$. Moreover, mutational profiles of indolent or outwardly stable CLL cases show that the presence of CLL clones bearing driver mutations do not have to correspond directly with disease progression. Therefore, simple mutation acquisition does not necessarily lead to immediate disease progression; nevertheless, accumulating changes precede the manifestation of disease activity. In addition, clonal evolution can occur in the absence of adverse prognostic factors such as the presence of high-risk cytogenetic alterations or unmutated IGHV. In fact, the acquisition of mutations can happen in the absence of any FISH alterations (P35 or P45) as well as in IGHV-mutated CLLs (P46). Unfortunately, analysis of genomic changes does not fully explain the transformation to a more aggressive stage in all CLL patients (P40). It was reported that epigenetic changes could also fuel CLL evolution during disease progression [6]. Understanding CLL evolution from the time of diagnosis to therapy need may be essential to gain insight into the process of transformation from the initial inactive form to later more aggressive stages. Although white blood cells (WBC) count during disease course is more feasible than performing NGS studies, we have observed that the acquisition of genomic alterations does not have to simply correspond with an increase of WBC (P4 or 35). Then, genomic analysis should be made in larger longitudinal-based cohort studies in order to evaluate how to predict disease activation in CLL. On the other hand, to understand the genomic changes underlying CLL relapse, mutational analysis at the time of diagnosis may be irrelevant as additional aberrations may appear during time and clonal shifts are likely to happen. Such analysis should be done before therapy intervention to monitor tumoral clones that are responsible for CLL relapse.

Acknowledgements The research leading to these results has mainly received funding from the European Union Seventh Framework Programme [FP7/2007-2013] under Grant Agreement no 306242-NGSPTL. In addition, this work was supported by grants from the Spanish Fondo de Investigaciones Sanitarias PI15/01471, PI18/01500, Instituto de Salud Carlos III (ISCIII), European Regional Development Fund (ERDF) "Una manera de hacer Europa", "Consejería de Educación, Junta de Castilla y León" (SA085U16), "Proyectos de Investigación del SACYL", Spain: GRS 994/A/14, BIO/SA10/14, BIO/SA31/13, GRS 1172/A/15,"Fundación Memoria Don Samuel Solórzano Barruso", by grants (RD12/0036/0069) from Red Temática de Investigación Cooperativa en Cáncer (RTICC), Centro de Investigación Biomédica en Red de Cáncer (CIBERONC) CB16/12/00233 and USAL "Programa XIII". M. Hernández-Sánchez is supported by FEHH-Janssen ("Sociedad Española de Hematología y Hemoterapia"). M Quijada-Álamo is supported by an "Ayuda Predoctoral de la Junta de Castilla y León" (JCYL-EDU/529/2017). We are grateful to I. Rodríguez, S. González, T.Prieto, M. Á. Ramos, A. Martín, A. Díaz, A. Simón, M.del Pozo, V. Gutiérrez and S. Pujante from Centro de Investigación del Cáncer, Salamanca, for their technical assistance. D. Tamborero is supported by project SAF2015-74072-JUN, which is funded by the Agencia Estatal de Investigación (AEI) and Fondo Europeo de Dearrollo Regional (FEDER). This work was supported by Seventh Framework Programme (NGS-PTL/2012-2015/no.306242) and Ministry of Education, Youth and Sports (2013-2015, no. 7E13008); by the Ministry of Education, Youth and Sports of the Czech Republic under the CEITEC 2020 project (LQ1601); by the Ministry of Health, Czech Republic - conceptual development of research organization (FNBr, 65269705); by the Specific University Research (nr. MUNI/A/0968/2017) provided by MEYS; and by the project CZ.02.1.01/0.0/0.0/16_013/0001634 National Center for Medical Genomic - modernization of infrastructure and research of genetic variation in the population, funded by OP RDE. We acknowledge the CF Genomics CEITEC MU supported by the NCMG research infrastructure (LM2015091 funded by MEYS CR) for their support with obtaining the scientific data presented in this paper. We acknowledge S. Takacova from CEITEC MU for her help with the sample selection and processing.

Authorship contributions MHS designed the experiment, analyzed data, and wrote the manuscript; JK designed the experiment, performed validation analysis and wrote the manuscript; AER performed sample selection and contributed to the interpretation of the results; LR and KP performed biostatistical analysis; DT performed bioinformatics analysis; MA, KPl, and RB performed sample selection; NT and MQ contributed to the interpretation of the results, VB performed validation analysis; AAM, MD, and AGC provided clinical data; NLB performed bioinformatics analysis; JMHR and SP designed the experiment and wrote the manuscript. All authors revised the manuscript.

\section{Compliance with ethical standards}

Conflict of interest The authors declare that they have no conflict of interest.

Open Access This article is licensed under a Creative Commons Attribution 4.0 International License, which permits use, sharing, adaptation, distribution and reproduction in any medium or format, as long as you give appropriate credit to the original author(s) and the source, provide a link to the Creative Commons license, and indicate if 
changes were made. The images or other third party material in this article are included in the article's Creative Commons license, unless indicated otherwise in a credit line to the material. If material is not included in the article's Creative Commons license and your intended use is not permitted by statutory regulation or exceeds the permitted use, you will need to obtain permission directly from the copyright holder. To view a copy of this license, visit http://creativecommons. org/licenses/by/4.0/.

\section{References}

1. Landau DA, Wu CJ. Chronic lymphocytic leukemia: molecular heterogeneity revealed by high-throughput genomics. Genome Med. 2013;5:47.

2. Puente XS, Bea S, Valdes-Mas R, Villamor N, Gutierrez-Abril J, Martin-Subero JI, et al. Non-coding recurrent mutations in chronic lymphocytic leukaemia. Nature. 2015;526:519-24.

3. Landau DA, Tausch E, Taylor-Weiner AN, Stewart C, Reiter JG, Bahlo $\mathrm{J}$, et al. Mutations driving CLL and their evolution in progression and relapse. Nature. 2015;526:525-30.

4. Ljungstrom V, Cortese D, Young E, Pandzic T, Mansouri L, Plevova $\mathrm{K}$, et al. Whole-exome sequencing in relapsing chronic lymphocytic leukemia: clinical impact of recurrent RPS15 mutations. Blood. 2016;127:1007-16.

5. Burger JA, Landau DA, Taylor-Weiner A, Bozic I, Zhang H, Sarosiek K, et al. Clonal evolution in patients with chronic lymphocytic leukaemia developing resistance to BTK inhibition. Nat Commun. 2016;7:11589.

6. Smith EN, Ghia EM, DeBoever CM, Rassenti LZ, Jepsen K, Yoon KA, et al. Genetic and epigenetic profiling of CLL disease progression reveals limited somatic evolution and suggests a relationship to memory-cell development. Blood Cancer J. 2015;5:e303.
7. Tamborero D, Rubio-Perez C, Deu-Pons J, Schroeder MP, Vivancos A, Rovira A, et al. Cancer Genome Interpreter annotates the biological and clinical relevance of tumor alterations. Genome Med. 2018;10:25.

8. Hallek M, Cheson BD, Catovsky D, Caligaris-Cappio F, Dighiero $\mathrm{G}$, Dohner $\mathrm{H}$, et al. iwCLL guidelines for diagnosis, indications for treatment, response assessment, and supportive management of CLL. Blood. 2018;131:2745-60.

9. Kubesova B, Pavlova S, Malcikova J, Kabathova J, Radova L, Tom N, et al. Low-burden TP53 mutations in chronic phase of myeloproliferative neoplasms: association with age, hydroxyurea administration, disease type and JAK2 mutational status. Leukemia. 2018;32:450-61.

10. Messina M, Del Giudice I, Khiabanian H, Rossi D, Chiaretti S, Rasi S, et al. Genetic lesions associated with chronic lymphocytic leukemia chemo-refractoriness. Blood. 2014;123:2378-88.

11. Van Damme V, Govaerts E, Nackaerts K, Dooms C, Wauters I, Vansteenkiste J. Clinical factors predictive of long-term survival in advanced non-small cell lung cancer. Lung Cancer. 2013;79:73-6.

12. Cazzola M. IDH1 and IDH2 mutations in myeloid neoplasms-novel paradigms and clinical implications. Haematologica. 2010;95:1623-7.

13. Mansouri L, Sutton LA, Ljungstrom V, Bondza S, Arngarden L, Bhoi S, et al. Functional loss of IkappaBepsilon leads to NFkappaB deregulation in aggressive chronic lymphocytic leukemia. J Exp Med. 2015;212:833-43.

14. Rossi D, Fangazio M, Rasi S, Vaisitti T, Monti S, Cresta S, et al. Disruption of BIRC3 associates with fludarabine chemorefractoriness in TP53 wild-type chronic lymphocytic leukemia. Blood. 2012;119:2854-62.

15. Zenz T, Eichhorst B, Busch R, Denzel T, Habe S, Winkler D, et al. TP53 mutation and survival in chronic lymphocytic leukemia. J Clin Oncol. 2010;28:4473-9.

\title{
Male sex and the pattern of recurrent myeloid mutations are strong independent predictors of blood transfusion intensity in patients with myelodysplastic syndromes
}

\author{
Jenny Rydén ${ }^{1,2} \cdot$ Gustaf Edgren $^{3,4} \cdot$ Mohsen Karimi $^{1} \cdot$ Gunilla Walldin $^{1} \cdot$ Magnus Tobiasson $^{1,2} \cdot$ Agneta Wikman $^{5,6}$. \\ Eva Hellström-Lindberg ${ }^{1,2}$. Petter Höglund ${ }^{1,5}$
}

Electronic supplementary material The online version of this article (https://doi.org/10.1038/s41375-018-0256-0) contains supplementary material, which is available to authorized users.

$\triangle$ Petter Höglund

Petter.Hoglund@ki.se

1 Center for Hematology and Regenerative Medicine, Department of Medicine, Karolinska Institutet, Stockholm, Sweden

2 Hematology Center, Karolinska University Hospital, Stockholm, Sweden
Received: 5 January 2018 / Revised: 23 July 2018 / Accepted: 7 August 2018 / Published online: 28 September 2018

(c) Springer Nature Limited 2018

3 Department of Medical Epidemiology and Biostatistics, Karolinska Institutet, Stockholm, Sweden

4 Department of Cardiology, Södersjukhuset, Stockholm, Sweden

Department of Clinical Immunology and Transfusion Medicine, Karolinska University Hospital, Stockholm, Sweden

6 Department of Laboratory Medicine, Karolinska University Hospital, Stockholm, Sweden 
Patients with myelodysplastic syndromes (MDS) frequently receive large numbers of blood transfusions due to inefficient hematopoiesis [1,2], with 94 and $48 \%$ of MDS patients receiving more than one red cell and platelet unit respectively during the course of their disease [3, 4]. Transfusion dependency in MDS is associated with reduced survival and significant impairment in quality of life $[1,4,5]$. Despite the extensive use of blood products in MDS, little is known about the clinical and disease-specific factors that drive red cell and platelet transfusion need in these patients. In this study, we added mutational patterns to clinical data and transfusion information in a large cohort of MDS patients to investigate predictors of transfusion intensity and the association between transfusion patterns and survival.

A retrospective cohort of 309 consecutive patients from the MDS biobank at the Karolinska University Hospital, Stockholm, Sweden, diagnosed between 2003 and 2013, met the inclusion criteria of MDS or MDS/myeloproliferative neoplasm according to the revised World Health Organization (WHO) 2008 classification (Supplementary Figure 1). Clinical and laboratory data for each patient, including mutational status at diagnosis, was linked to their transfusion history, comprehensively retrieved from the local transfusion database (Supplementary Table 1). Patients were followed with regard to transfusion history from the date of diagnosis until the date of death, allogeneic stem cell transplantation (SCT), or 1 December 2013, whichever occurred first. The mutations were categorized according to their involvement in histone modulation, DNA methylation, DNA splicing, cohesin complex, and signaling or transcriptional regulation, in analogy with previously published data [6-8]. TP53 mutations were considered separately, and 'other mutations', occurring in only a few patients each, were grouped together (Supplementary Table 2). All patients were treated according to the Nordic guidelines (www.nmds.org) which follow the European Recommendations for treatment of MDS [9] (Supplementary Table 3). The study was approved by the Regional Ethical Committee in Stockholm.

The cohort received a total of 11,350 red cell and 1956 platelet units over 777 person-years of follow-up, corresponding to an overall transfusion intensity (TI) of 14.6 and 2.5 units/person-year for red cell and platelet units, respectively (Table 1). These data were fitted in Poisson regression analyses estimating the relative TI associated with a number of considered clinical parameters, expressed as incidence rate ratios (IRRs). High and very high IPSS-R (International prognostic scoring system-revised) and male sex were found to be significantly associated with a high red cell TI (IRR 2.0, 95\% confidence interval (CI) 1.9-2.1; IRR 2.7, 95\% CI 2.6-3.0; and IRR 2.0, 95\% CI 1.6-1.8, respectively) (Table 1; Fig. S2A). Mutations in histone modulators, signaling factors, and transcriptional regulators were positively associated with red cell TI, while mutations in DNA methylation, splice factors, cohesion factors, TP53 mutations, and 'other' mutations were negatively associated with red cell TI (Table 1; Fig. S2A). For platelet TI, male sex and very high IPSS-R risk were significantly associated with high platelet TI (IRR 2.0, 95\% CI 1.8-2.3; and IRR 2.3, 95\% CI 1.9-2.7, respectively) (Table 1; Fig. S2B). Mutations in histone modulators, signaling factors, and transcriptional regulators were positively associated with platelet TI, while mutations in DNA methylation, splice factors, cohesion factors, and 'other' mutations were associated with lower TI (Table 1; Fig. S2B).

The analysis of TI and association with patient survival was conducted using a time-dependent Cox proportional hazards model, estimating hazard ratios of death, with $95 \%$ CIs, censoring patients by date of allogeneic SCT or end of follow-up. The number of red cell and platelet transfusions in the past year, i.e. TI, was significantly and independently associated with an increased risk of death. In fact, TI was the only factor that remained strongly significant after adjusting for age, sex, IPSS-R, and most mutations in a multivariable analysis (Fig. 1). Interestingly, histone modulator mutations, including ASXL1, which was positively linked to TI (Table 1), was negatively associated with risk of death in the same multivariable analysis (Fig. 1).

Given the importance of well-known MDS mutations for hematopoietic function as well as for disease prognosis, links between mutations and TI are not surprising. It was expected that transcription factor mutations should be associated with a higher TI, as these mutations also drive poorer overall disease outcome $[8,10]$. More unexpected was the association between mutations in histone modulators and increased TI of both red cells and platelets. When other risk factors and transfusion need were accounted for, histone modulator mutations were no longer associated with poor survival, suggesting that these mutations are more involved in mechanisms underlying ineffective hematopoiesis than disease progression. Furthermore, splice factor and DNA methylation mutations were associated with reduced TI. The former group, dominated by lower-risk MDS with ring sideroblasts and SF3B1 mutations, was protected from transfusion need early on in disease course, but lost this effect after approximately 2-3 years (data not shown). This corresponds well with the fact that these patients receive erythropoietin-stimulating agents (ESA) as first-line treatment (Supplementary Table 3) and have a high probability of response during the first years [11, 12]. Finally, the seemingly 'protective' effect of TP53 mutations on TI may have two explanations. The first is that TP53-mutated higher-risk MDS often have a good but short-lasting 
Table 1 Predictors of red cell and platelet transfusion intensity

\begin{tabular}{|c|c|c|c|c|c|c|c|}
\hline & \multirow[t]{2}{*}{$\begin{array}{l}\text { No. of } \\
\text { patients }\end{array}$} & \multirow[t]{2}{*}{$\begin{array}{l}\text { Red cell } \\
\text { transfusions/ } \\
\text { person-year }\end{array}$} & \multicolumn{2}{|c|}{$\begin{array}{l}\text { Multivariable model for } \\
\text { red cell transfusion } \\
\text { intensity }\end{array}$} & \multirow[t]{2}{*}{$\begin{array}{l}\text { Platelet } \\
\text { transfusions/ } \\
\text { person-year }\end{array}$} & \multicolumn{2}{|c|}{$\begin{array}{l}\text { Multivariable model for } \\
\text { platelet transfusion } \\
\text { intensity }\end{array}$} \\
\hline & & & $\begin{array}{l}\text { Incidence rate } \\
\text { ratio }(95 \% \mathrm{CI})\end{array}$ & $P$ value $^{\mathrm{a}}$ & & $\begin{array}{l}\text { Incidence rate } \\
\text { ratio }(95 \% \mathrm{CI})\end{array}$ & $P$ value \\
\hline Total cohort & 309 & $11350 / 777=14.6$ & & & $1956 / 777=2.5$ & & \\
\hline \multicolumn{8}{|l|}{ Sex } \\
\hline Female & 123 & $3753 / 343=10.9$ & 1.0 (ref) & & $651 / 343=1.9$ & 1.0 (ref) & \\
\hline Male & 186 & $7597 / 434=17.5$ & $1.7(1.6-1.8)$ & $<0.001$ & $1305 / 434=3.0$ & $2.0(1.8-2.3)$ & $<0.001$ \\
\hline \multicolumn{8}{|l|}{ Age at diagnosis, years } \\
\hline$<65$ & 74 & $2068 / 163=12.7$ & $0.8(0.7-0.8)$ & $<0.001$ & $584 / 163=3.6$ & $0.9(0.8-1.0)$ & 0.087 \\
\hline $65-74$ & 100 & $4262 / 287=14.9$ & 1.0 (ref) & & $912 / 287=3.2$ & 1.0 (ref) & \\
\hline$>74$ & 135 & $5020 / 326=15.4$ & $0.9(0.8-0.9)$ & $<0.001$ & $460 / 326=1.4$ & $0.3(0.3-0.4)$ & $<0.001$ \\
\hline \multicolumn{8}{|l|}{ IPSS-R } \\
\hline Intermediate & 71 & $2503 / 171=14.6$ & 1.0 (ref) & & $687 / 171=4.0$ & 1.0 (ref) & \\
\hline Very low & 51 & $1771 / 154=11.5$ & $0.8(0.7-0.8)$ & $<0.001$ & $136 / 154=0.9$ & $0.3(0.3-0.4)$ & $<0.001$ \\
\hline Low & 95 & $3105 / 319=9.7$ & $0.7(0.7-0.7)$ & $<0.001$ & $251 / 319=0.8$ & $0.2(0.2-0.2)$ & $<0.001$ \\
\hline High & 55 & $2615 / 93=28.1$ & $2.0(1.9-2.1)$ & $<0.001$ & $609 / 93=6.5$ & $1.1(0.9-1.2)$ & 0.099 \\
\hline Very high & 31 & $878 / 24=36.6$ & $2.7(2.6-3.0)$ & $<0.001$ & $217 / 24=9.0$ & $2.3(1.9-2.7)$ & $<0.001$ \\
\hline \multicolumn{8}{|l|}{ WHO classification } \\
\hline $\begin{array}{l}\text { Lower risk without ring } \\
\text { sideroblasts }\end{array}$ & 86 & $3293 / 247=13.3$ & $\mathrm{n} / \mathrm{a}$ & $\mathrm{n} / \mathrm{a}$ & $364 / 247=1.5$ & $\mathrm{n} / \mathrm{a}$ & $\mathrm{n} / \mathrm{a}$ \\
\hline $\begin{array}{l}\text { Lower risk with ring } \\
\text { sideroblasts }\end{array}$ & 63 & $1829 / 208=8.8$ & $\mathrm{n} / \mathrm{a}$ & $\mathrm{n} / \mathrm{a}$ & $52 / 208=0.3$ & $\mathrm{n} / \mathrm{a}$ & $\mathrm{n} / \mathrm{a}$ \\
\hline CMML & 35 & $1055 / 86=12.3$ & $\mathrm{n} / \mathrm{a}$ & $\mathrm{n} / \mathrm{a}$ & $353 / 86=4.1$ & $\mathrm{n} / \mathrm{a}$ & $\mathrm{n} / \mathrm{a}$ \\
\hline RAEB $1-2$ & 116 & $4747 / 215=22.1$ & $\mathrm{n} / \mathrm{a}$ & $\mathrm{n} / \mathrm{a}$ & $1141 / 215=5.3$ & $\mathrm{n} / \mathrm{a}$ & $\mathrm{n} / \mathrm{a}$ \\
\hline MDS-MPN, UNS & 7 & $414 / 14=29.6$ & $\mathrm{n} / \mathrm{a}$ & $\mathrm{n} / \mathrm{a}$ & $44 / 14=3.1$ & $\mathrm{n} / \mathrm{a}$ & $\mathrm{n} / \mathrm{a}$ \\
\hline \multicolumn{8}{|l|}{ Bone marrow cellularity $(\%)$} \\
\hline$<30$ & 15 & $348 / 30=11.6$ & $0.5(0.4-0.5)$ & $<0.001$ & $50 / 30=1.7$ & $0.2(0.2-0.3)$ & $<0.001$ \\
\hline $30-50$ & 95 & $3224 / 220=14.7$ & 1.0 (ref) & & $416 / 220=1.9$ & 1.0 (ref) & \\
\hline$>50$ & 174 & $6304 / 443=14.2$ & $0.9(0.9-0.9)$ & $<0.001$ & $1361 / 443=3.1$ & $1.1(1.0-1.2)$ & 0.078 \\
\hline \multicolumn{8}{|l|}{ Mutation status ${ }^{\mathrm{b}}$} \\
\hline $\begin{array}{l}\text { Histone modulator mutations } \\
(A S X L 1, M L L, E Z H 2)\end{array}$ & 42 & $1926 / 84=22.9$ & $1.8(1.7-1.9)$ & $<0.001$ & $485 / 84=5.8$ & $3.3(2.9-3.8)$ & $<0.001$ \\
\hline $\begin{array}{l}\text { DNA methylation mutations } \\
\text { (TET2, DNMT3A, IDH1/2) }\end{array}$ & 98 & $3518 / 248=14.2$ & $0.9(0.8-0.9)$ & $<0.001$ & $634 / 248=2.6$ & $0.7(0.6-0.8)$ & $<0.001$ \\
\hline $\begin{array}{l}\text { Splice factor mutations (SF3B1, } \\
S R S F 2, U A F 2, P R P F 40 B \text {, } \\
Z R S R 2, S F 3 A 1, S F 1, U 2 A F 1)\end{array}$ & 125 & $4431 / 340=13.0$ & $0.9(0.8-0.9)$ & $<0.001$ & $762 / 340=2.2$ & $0.6(0.5-0.7)$ & $<0.001$ \\
\hline $\begin{array}{l}\text { Cohesin factor mutations } \\
(S T A G 1 / 2, S M C 1 A, S M C 3, \\
R A D 21, P D S 5 B)\end{array}$ & 10 & $349 / 23=15.2$ & $0.4(0.4-0.5)$ & $<0.001$ & $76 / 23=3.3$ & $0.7(0.5-0.9)$ & $<0.05$ \\
\hline $\begin{array}{l}\text { Signaling factor mutations } \\
(J A K 2, M P L, C B L, N R A S, \\
K R A S, K I T, S H 2 B 3, F L T 3, \\
W T 1)\end{array}$ & 38 & $1980 / 104=19.0$ & $1.5(1.4-1.6)$ & $<0.001$ & $506 / 104=4.9$ & $2.4(2.1-2.7)$ & $<0.001$ \\
\hline $\begin{array}{l}\text { Transcription factor mutations } \\
\text { (RUNX1, BCOR, GATA1/2/3, } \\
\text { ETV6, CEBPA) }\end{array}$ & 30 & $1064 / 46=23.1$ & $1.2(1.1-1.3)$ & $<0.001$ & $495 / 46=10.8$ & $2.9(2.5-3.3)$ & $<0.001$ \\
\hline TP53 mutation & 25 & $641 / 38=16.9$ & $0.7(0.6-0.8)$ & $<0.001$ & $164 / 38=4.3$ & $1.0(0.9-1.2)$ & 0.795 \\
\hline Other mutations (NF1, SETBP1, & 22 & $520 / 49=10.6$ & $0.6(0.6-0.7)$ & $<0.001$ & $149 / 49=3.0$ & $0.3(0.2-0.3)$ & $<0.001$ \\
\hline
\end{tabular}

$C R E B B P$ among others) 
Table 1 (continued)

\begin{tabular}{|c|c|c|c|c|c|}
\hline \multirow[t]{2}{*}{$\begin{array}{l}\text { No. of } \\
\text { patients }\end{array}$} & \multirow[t]{2}{*}{$\begin{array}{l}\text { Red cell } \\
\text { transfusions/ } \\
\text { person-year }\end{array}$} & \multicolumn{2}{|c|}{$\begin{array}{l}\text { Multivariable model for } \\
\text { red cell transfusion } \\
\text { intensity }\end{array}$} & \multirow[t]{2}{*}{$\begin{array}{l}\text { Platelet } \\
\text { transfusions/ } \\
\text { person-year }\end{array}$} & $\begin{array}{l}\text { Multivariable model for } \\
\text { platelet transfusion } \\
\text { intensity }\end{array}$ \\
\hline & & $\begin{array}{l}\text { Incidence rate } \\
\text { ratio }(95 \% \mathrm{CI})\end{array}$ & $P$ value $^{\mathrm{a}}$ & & $\begin{array}{l}\text { Incidence rate } \\
\text { ratio }(95 \% \mathrm{CI})\end{array}$ \\
\hline
\end{tabular}

Number of mutations

\begin{tabular}{llllllll}
0 or SF3B1 & 132 & $4766 / 362=13.2$ & $\mathrm{n} / \mathrm{a}$ & $\mathrm{n} / \mathrm{a}$ & $592 / 362=1.6$ & $\mathrm{n} / \mathrm{a}$ & $\mathrm{n} / \mathrm{a}$ \\
$1-2$, not SF3B1 & 54 & $2268 / 121=18.7$ & $\mathrm{n} / \mathrm{a}$ & $\mathrm{n} / \mathrm{a}$ & $403 / 121=3.3$ & $\mathrm{n} / \mathrm{a}$ & $\mathrm{n} / \mathrm{a}$ \\
$\geq 2$ Independent of SF3B1 status & 123 & $4316 / 294=14.7$ & $\mathrm{n} / \mathrm{a}$ & $\mathrm{n} / \mathrm{a}$ & $961 / 294=3.3$ & $\mathrm{n} / \mathrm{a}$ & $\mathrm{n} / \mathrm{a}$ \\
\hline
\end{tabular}

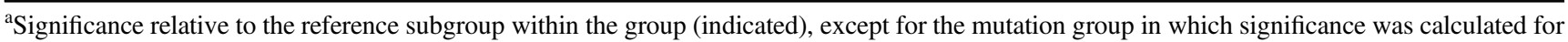
each mutational subgroup

${ }^{b}$ Mutations were categorized according to their involvement in histone modification, DNA methylation, splicing, cohesin complex, signaling, transcriptional regulation, TP53 mutation, and 'other mutations'

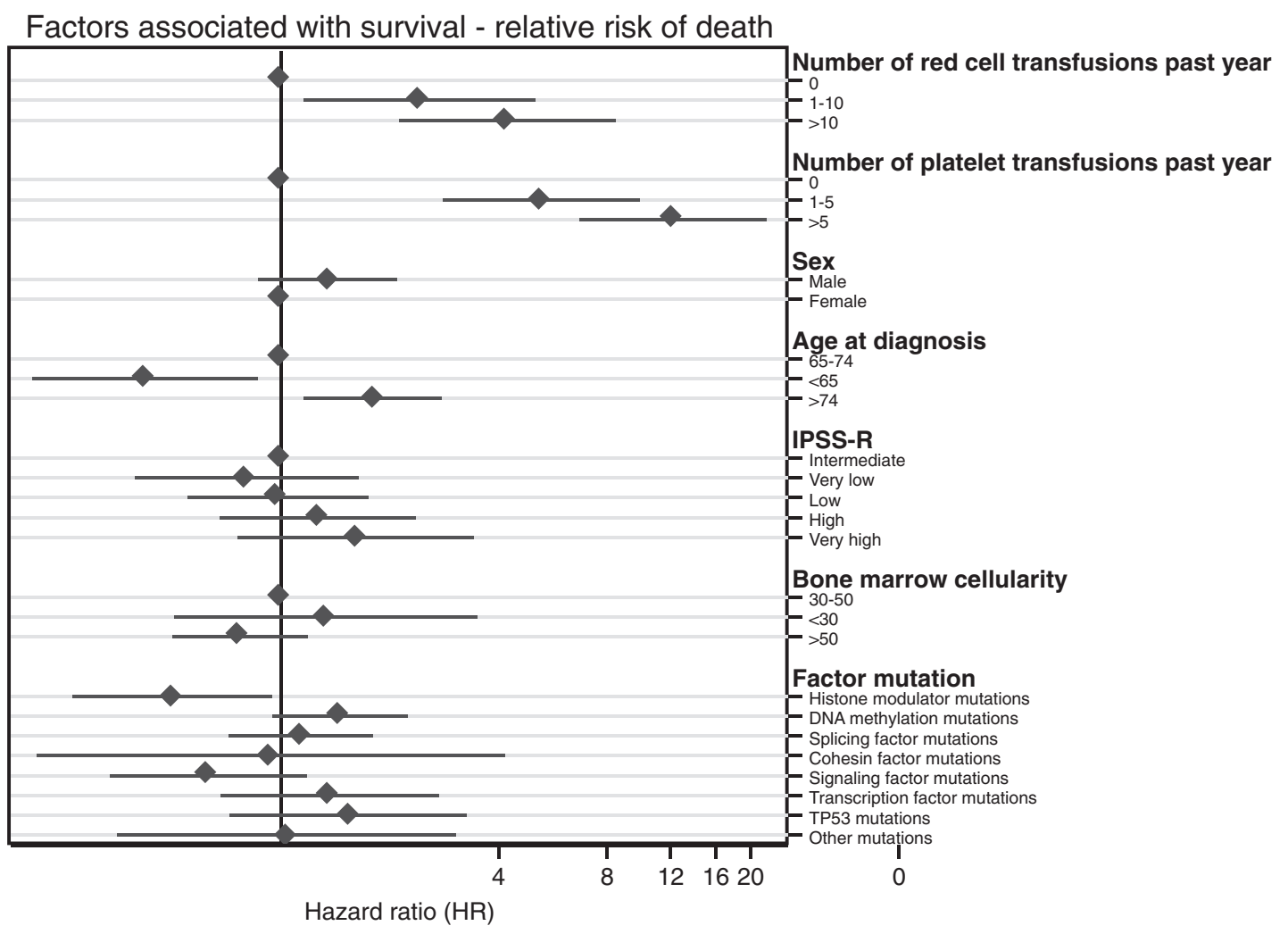

Fig. 1 Forest plot of the association between transfusion intensity and survival. The number of red cell and platelet transfusions the past year was significantly associated with an increased risk of death (IRR $>1$ ). IPSS-R International prognostic scoring system-revised

response to hypomethylating agents (HMAs) [13], and the other that once the disease relapses, overall survival is poor, and usually without a long palliative phase with multiple transfusions.

The question is to what extent the association between TI and mutational status is influenced by MDS therapy. The only therapy that permanently affects transfusion need in MDS is successful allogeneic SCT, hence all patients were censored at SCT. Other therapies, with the possible and rare exception of anti-thymocyte globulin, are associated with relapse, usually within 1-3 years. The majority of the lower-risk MDS patients who are anemic receive first-line 
treatment with ESA according to guidelines, and relapse with transfusion dependency after a median of approximately 25 months. In our cohort, the vast majority of these patients received chronic transfusion therapy as second-line treatment. Thus, since ESA treatment primarily is given early in the course of the disease, its influence on TI over time and during the last year, an important piece of data in our study, is limited. Secondly, we noted that the group of patients receiving ESA only was approximately twice as large in the splice factor mutation/DNA methylation groups compared to the group of patients with histone modulator mutations (Supplementary Table 3). This indicates that the response to ESA treatment is better in the former groups, but also that a lost response to ESA in these patients is usually not associated with progression to higher-risk MDS motivating treatment with, e.g., HMA.

Patients with histone modulator mutations constitute a more heterogeneous group with regard to risk and treatment (Supplementary Table 3), hence not considered to be influenced by any particular therapeutic option. In addition to ESAs and SCT, there was only one additional patient group of size; those treated with HMA $(n=70)$. It is well documented also in our own cohort that the overall response rate to HMA is less than $50 \%$ and that the frequency of complete and partial remission is around $30 \%$ [6, 14]. The median response duration is less than 1 year and most patients in our cohort received palliative treatment after relapse but with complete follow-up with regard to transfusions and survival. In conclusion, except for the early protective effect of ESA treatment in splice factor mutated lower-risk patients, we believe that treatment is not a major confounding variable in this cohort, since a major part of the observation period consists of time without response or treatment.

A novel and unexpected finding in our study was that male patients presented with around twice the TI for both red cell and platelet units, compared to female patients. It is known from earlier studies that male MDS patients have overall poorer survival [15]. Indeed, we found a poorer survival of males in unadjusted analysis (data not shown) and it is possible that male patients simply had a more aggressive disease, requiring more transfusions. However, male sex was strongly associated with transfusion need even when adjusting for several clinical parameters, including mutations, indicating that the effect is not entirely driven by disease biology. Furthermore, there were no relevant differences in the pre-transfusion hemoglobin levels between males and females, nor could the transfusion intensity be explained by the generally larger blood volume in males (data not shown). This unexpected male bias needs further investigation.

We conclude from our study that TI is a much stronger predictor for survival than previously recognized.
Furthermore, the strong association between mutational profile and TI indicates an important role for specific mutations and gene pathways in the control of hematopoiesis. Speculative mechanisms may include yet unknown effects of specific mutations on the bone marrow niche, affecting circulation and survival of transfused cells. Alternatively, mutations could affect the lifespan of the patient's own red blood cells and platelets, leading to an increased transfusion demand for this reason. Experimental studies will have to be designed in order to investigate these alternatives.

Acknowledgements This study was supported by independent grants to PH and EH-L from the Swedish Research Council, the Swedish Cancer Society, Radiumhemmets Forskningsfonder and the Stockholm City Council. JR was supported by a research residence grant from the Stockholm City Council. We thank all members of the PH, EH-L, and GE groups for discussions.

\section{Compliance with ethical standards}

Conflict of interest The authors declare that they have no conflict of interest.

\section{References}

1. Cazzola M, Malcovati L. Myelodysplastic syndromes--coping with ineffective hematopoiesis. N Eng J Med. 2005;352:536-8.

2. Zhao J, Ryden J, Wikman A, Norda R, Stanworth SJ, Hjalgrim H. et al. Blood use in hematologic malignancies: a nationwide overview in Sweden between 2000 and 2010. Transfusion. 2018;58:390-401.

3. Ramsey SD, McCune JS, Blough DK, McDermott CL, Beck SJ, Lopez JA, et al. Patterns of blood product use among patients with myelodysplastic syndrome. Vox Sang. 2012;102:331-7.

4. Platzbecker U, Hofbauer LC, Ehninger G, Holig K. The clinical, quality of life, and economic consequences of chronic anemia and transfusion support in patients with myelodysplastic syndromes. Leuk Res. 2012;36:525-36.

5. de Swart L, Smith A, Johnston TW, Haase D, Droste J, Fenaux P, et al. Validation of the revised international prognostic scoring system (IPSS-R) in patients with lower-risk myelodysplastic syndromes: a report from the prospective European LeukaemiaNet MDS (EUMDS) registry. Br J Haematol. 2015;170:372-83.

6. Tobiasson M, McLornan DP, Karimi M, Dimitriou M, Jansson M, Ben Azenkoud A, et al. Mutations in histone modulators are associated with prolonged survival during azacitidine therapy. Oncotarget. 2016;7:22103-15.

7. Karimi M, Nilsson C, Dimitriou M, Jansson M, Matsson H, Unneberg $\mathrm{P}$, et al. High-throughput mutational screening adds clinically important information in myelodysplastic syndromes and secondary or therapy-related acute myeloid leukemia. Haematologica. 2015;100:e223-5.

8. Papaemmanuil E, Gerstung M, Malcovati L, Tauro S, Gundem G, Van Loo P, et al. Clinical and biological implications of driver mutations in myelodysplastic syndromes. Blood. 2013;122:361627. quiz 99

9. Malcovati L, Hellstrom-Lindberg E, Bowen D, Ades L, Cermak J, Del Canizo C, et al. Diagnosis and treatment of primary myelodysplastic syndromes in adults: recommendations from the European LeukemiaNet. Blood. 2013;122:2943-64. 
10. Malcovati L, Papaemmanuil E, Ambaglio I, Elena C, Galli A, Della Porta MG, et al. Driver somatic mutations identify distinct disease entities within myeloid neoplasms with myelodysplasia. Blood. 2014;124:1513-21.

11. Jadersten M, Malcovati L, Dybedal I, Della Porta MG, Invernizzi R, Montgomery SM, et al. Erythropoietin and granulocyte-colony stimulating factor treatment associated with improved survival in myelodysplastic syndrome. J Clin Oncol. 2008;26:3607-13.

12. Garelius HK, Johnston WT, Smith AG, Park S, de Swart L, Fenaux P. et al. Erythropoiesis-stimulating agents significantly delay the onset of a regular transfusion need in nontransfused patients with lower-risk myelodysplastic syndrome. J Intern Med. 2017;281:284-99.
13. Welch JS, Petti AA, Miller CA, Fronick CC, O'Laughlin M, Fulton RS, et al. TP53 and decitabine in acute myeloid leukemia and myelodysplastic syndromes. N Eng J Med. 2016;375:202336.

14. Fenaux P, Mufti GJ, Hellstrom-Lindberg E, Santini V, Finelli C, Giagounidis A, et al. Efficacy of azacitidine compared with that of conventional care regimens in the treatment of higher-risk myelodysplastic syndromes: a randomised, open-label, phase III study. Lancet Oncol. 2009;10:223-32.

15. Malcovati L, Porta MG, Pascutto C, Invernizzi R, Boni M, Travaglino E, et al. Prognostic factors and life expectancy in myelodysplastic syndromes classified according to WHO criteria: a basis for clinical decision making. J Clin Oncol. 2005;23:7594603.

Leukemia (2019) 33:527-531

https://doi.org/10.1038/s41375-018-0258-y

Multiple myeloma gammopathies

\title{
Optimizing deep response assessment for AL amyloidosis using involved free light chain level at end of therapy: failure of the serum free light chain ratio
}

\author{
Eli Muchtar $\mathbb{D}^{1} \cdot$ Angela Dispenzieri $^{1} \cdot$ Nelson Leung $\mathbb{D}^{1,2} \cdot$ Martha Q. Lacy $^{1} \cdot$ Francis K. Buadi $^{1}$ - David Dingli ${ }^{1}$. \\ Suzanne R. Hayman ${ }^{1}$. Prashant Kapoor ${ }^{1}$ - Yi Lisa Hwa ${ }^{1}$ - Amie Fonder ${ }^{1}$ • Miriam Hobbs ${ }^{1}$ - Wilson Gonsalves ${ }^{1}$. \\ Taxiarchis V. Kourelis ${ }^{1} \cdot$ Rahma Warsame $^{1}$ - Stephen J. Russell ${ }^{1} \cdot$ John A. Lust ${ }^{1} \cdot$ Yi Lin $^{1} \cdot$ Ronald S. Go $\mathbb{D}^{1}$ \\ Steven R. Zeldenrust ${ }^{1} \cdot$ Robert A. Kyle $^{1} \cdot$ S. Vincent Rajkumar ${ }^{1} \cdot$ Shaji K. Kumar $^{1}{ }^{1} \cdot$ Morie A. Gertz $^{1}$
}

Received: 7 June 2018 / Revised: 9 August 2018 / Accepted: 14 August 2018 / Published online: 26 September 2018 (c) Springer Nature Limited 2018

The baseline level of involved free light chains (iFLC) is prognostic in light chain (AL) amyloidosis [1, 2]. A reduction in iFLC is associated with improved survival and organ response [1, 3, 4], making the serum free light chain (sFLC) assay the basis for the hematological response criteria [5]. Since AL amyloidosis is associated with a low burden of iFLC, different clinical outcomes can be seen at similar response depth. In patients who achieve normal sFLC ratio (sFLCR), high iFLC continues to predict a worse outcome compared to those with a normal iFLC [6].

Electronic supplementary material The online version of this article (https://doi.org/10.1038/s41375-018-0258-y) contains supplementary material, which is available to authorized users.

Morie A. Gertz

gertz.morie@mayo.edu

Division of Hematology, Mayo Clinic, Rochester, MN, USA

2 Division of Nephrology and Hypertension, Mayo Clinic, Rochester, MN, USA
This study explored the impact of depth of hematological response using sFLC assay parameterson organ response and survival.

The inclusion criteria were: (1) newly diagnosed AL amyloidosis within 90 days of diagnosis between 1/2002 and $8 / 2015$; (2) achievement of very good partial response (VGPR) or complete response (CR) to first-line therapy [5]; and (3) serial sFLC studies available. The Mayo Foundation Institutional Review Board approved the study. All patients gave written informed consent to have their medical records reviewed.

sFLC assay measures at the end of first-line therapy and at nadir iFLC after therapy completion were analyzed, and included: iFLC, the difference between involved-touninvolved light chains (dFLC) and sFLCR. Treatment was categorized as autologous stem cell transplant (ASCT, $n=248)$ or non-ASCT $(n=148)$. Day +100 from ASCT was considered the end of therapy for ASCT.

Survival was calculated using the Kaplan-Meier method. Hematological progression-free survival (HemPFS) was defined from diagnosis until hematological progression or 
death, where hematological progression was defined according to consensus criteria [7] or upon initiation of second-line therapy. Overall survival (OS) was defined as the time from diagnosis until death from any cause. Multivariate analysis was performed using the Cox Proportional Hazards model.

The median age was 59. Men comprised 59\%. The kidney was the most commonly involved organ at $69 \%$ (Supplementary Table 1). Complete response was achieved in $63 \%$ of patients, $37 \%$ achieved a VGPR. At the end of first-line treatment the median iFLC was $1.7 \mathrm{mg} / \mathrm{dL}$ (IQR, $1.1-2.9)$ and the median dFLC was $0.5 \mathrm{mg} / \mathrm{dL}$ (IQR, $0-1.3$ ). There was no difference between iFLC $(P=0.27)$ or dFLC $(P=0.5)$ between lambda or kappa light chain amyloidosis. sFLCR Normalization by the end of therapy was achieved in $70 \%$ of patients, and was more likely in lambda light chain disease $(75 \%$ vs. $55 \% ; P<0.001)$. Baseline characteristics based on iFLC level at the end of therapy is in Supplementary Table 2. The median nadir iFLC after treatment completion was $1.4 \mathrm{mg} / \mathrm{dL}$ (IQR 0.9-2.3) with a median dFLC of $0.3 \mathrm{mg} / \mathrm{dL}$ [IQR (-0.2) - 0.8)]. A normal sFLCR was achieved in $76 \%$ of patients at nadir iFLC. The median time from end of therapy to nadir iFLC was 3.9 months (IQR 2.8-12.6). The time to nadir iFLC was 7.3 months in ASCT patients and 1.8 months in non-ASCT patients $(P<0.001)$. Nadir iFLC was lower among ASCT patients (median $1.2 \mathrm{mg} / \mathrm{dL}$, IQR $0.8-1.8$ ) compared to non-ASCT patients (median $1.9 \mathrm{mg} / \mathrm{dL}$, IQR 1.3-3.0; $P<0.001)$. No differences between groups was seen in dFLC at time of nadir iFLC $(P=0.34)$ or in the frequency of sFLCR normalization $(P=0.28)$.

Organ response data was available for $93 \%$ of patients with cardiac and renal involvement and $97 \%$ of patients with hepatic involvement. In all three organs, iFLC $\leq 2 \mathrm{mg} /$ was associated with a higher organ response rate compared to $\mathrm{iFLC}>2 \mathrm{mg} / \mathrm{dL}$ (cardiac response $86 \%$ vs. $59 \%$, $P<0.001$; renal response $92 \%$ vs. $63 \%, P<0.001$; hepatic response $84 \%$ vs. $59 \%, P=0.03$ ). iFLC had a better organ response prediction over dFLC in all three organs (Supplementary Table 3). Normalization of sFLCR at the end of therapy did not predict for a higher organ response rate compared to patients with abnormal sFLCR ratio $(P=$ $0.33-0.88$ ).

At the end of therapy iFLC $\leq 2.0 \mathrm{mg} / \mathrm{dL}$ was associated with significantly longer hemPFS compared to patients with an $\mathrm{iFLC}>2 \mathrm{mg} / \mathrm{dL}$ (median 118 vs 56 months, $P<0.001$; Supplementary Fig. 1a). Lambda amyloidosis resulted in a larger difference in hemPFS between iFLC groups (median 118 vs 52 months, $P<0.001$; Supplementary Fig. 1b) comparted to kappa amyloidosis (median 117 vs. 76 months, $P=0.048$; Supplementary Fig. 1c). dFLC at $0.5 \mathrm{mg} / \mathrm{dL}$ had similar predictive value for hemPFS as iFLC at $2.0 \mathrm{mg} / \mathrm{dL}$ (median 117 vs. 56 months, $P<0.001$;
Supplementary Fig. 1d). Normalization of sFLCR had no impact on hemPFS compared to abnormal sFLCR $(P=$ 0.53; Supplementary Fig. 1e).

iFLC $\leq 1 \mathrm{mg} / \mathrm{dL}$ was associated with significantly longer hemPFS compared to patients with iFLC $>1 \mathrm{mg} / \mathrm{dL}$ (median 145 vs. 67 months, $P<0.001$; Supplementary Fig. 2a). By light chain isotype only lambda amyloidosis remained statistically significant in predicting hemPFS (median 145 vs. 65 months, $P=0.001$; Supplementary Fig. 2b), whereas kappa amyloidosis was not (median 117 vs. 82 months, $P=0.25$; Supplementary Fig. 2c).

The median follow-up of surviving patients was 89 months. iFLC $\leq 2 \mathrm{mg} / \mathrm{dL}$ by the end of first-line therapy predicted for improved OS compared to iFLC $>2 \mathrm{mg} / \mathrm{dL}$ (10-year OS $76 \%$ vs. $55 \%, P<0.001$; Fig. 1a). Only lambda light chain predicted for improved OS $(P<0.001$; Fig. 1b), while kappa light chain amyloidosis was not $(P=0.86$; Fig. 1c). iFLC $\leq 1 \mathrm{mg} / \mathrm{dL}$ was associated with longer OS compared to iFLC $>1 \mathrm{mg} / \mathrm{dL}$ (10-year OS $80 \%$ vs. $64 \%$; $P$ $=0.02)$. dFLC $\leq 0.5 \mathrm{mg} / \mathrm{dL}$ did not predict for longer survival compared to dFLC $>0.5 \mathrm{mg} / \mathrm{dL}(P=0.11$; Fig. $1 \mathrm{~d})$, even by subgrouping based on light chain isotype (both $P>0.2$ ). Patients who achieved normal sFLCR did not have a better OS compared to patients with abnormal sFLCR at end of therapy $(P=0.91$; Fig. 1e). HemPFS and OS by iFLC groups among CR and VGPR subgroups is in Supplementary Fig. 3.

We examined the performance of sFLC parameters at the end of therapy among patients with estimated glomerular filtration rate $<30 \mathrm{ml} / \mathrm{min} / 1.73 \mathrm{~m}^{2}(n=49)$. The median iFLC among these patients was $3.3 \mathrm{mg} / \mathrm{dL}$ and the median $\mathrm{dFLC}$ was $0.3 \mathrm{mg} / \mathrm{dL}$. Normalization of sFLCR occurred in $69 \%$ of patients, higher in lambda restricted disease compared to kappa restricted disease (79\% vs. $50 \%, P=0.04)$. There was no difference between lambda and kappa isotype groups in iFLC value (median 3.1 vs. $3.5 \mathrm{mg} / \mathrm{dL}$, respectively; $P=0.93$ ). No difference was seen in hemPFS when considering iFLC at $3 \mathrm{mg} / \mathrm{dL}$ (value close to median at this subgroup) $(P=0.9)$ or $\mathrm{dFLC}$ at $0.5 \mathrm{mg} / \mathrm{dL}(P=0.44)$. Similarly, no difference in OS was seen, either by using iFLC at $3 \mathrm{mg} / \mathrm{dL}(P=0.21)$ or $\mathrm{dFLC}$ at $0.5 \mathrm{mg} / \mathrm{dL}$ $(P=0.86)$.

Patients in whom nadir iFLC was reached $>12$ months from the end of therapy had a significantly longer hemPFS compared to patients in whom nadir iFLC was reached within 12 months from end of therapy (median 147 vs. 65 months, $P<0.001$; Supplementary Fig. 4a). There was also a difference in OS between groups (10-year OS $82 \%$ vs. $62 \%, P<0.001$; Supplementary Fig. 4b). In patients who underwent ASCT, hemPFS was significantly longer in patients with nadir iFLC reached $>12$ months from diagnosis compared to their counterparts (median 147 vs. 87 months; $\mathrm{P}=0.002$ ), as well as for those who received 

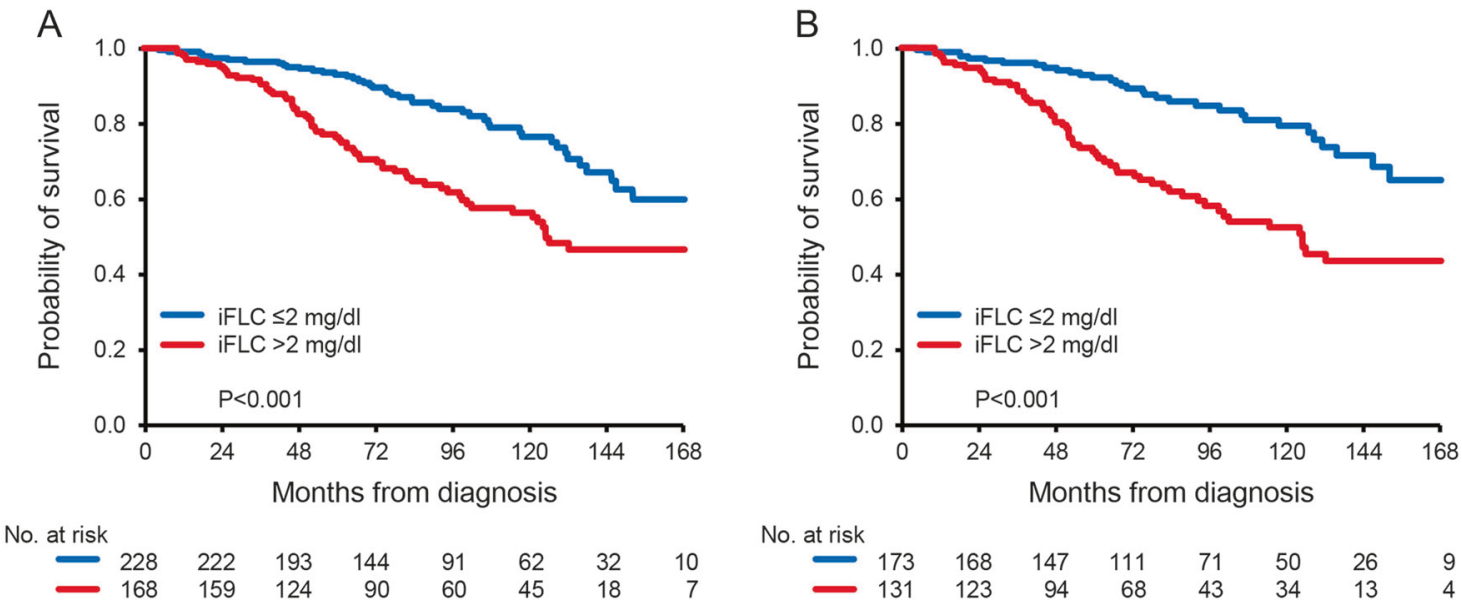

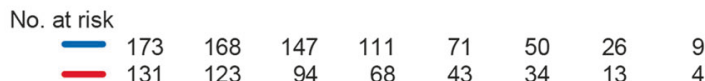
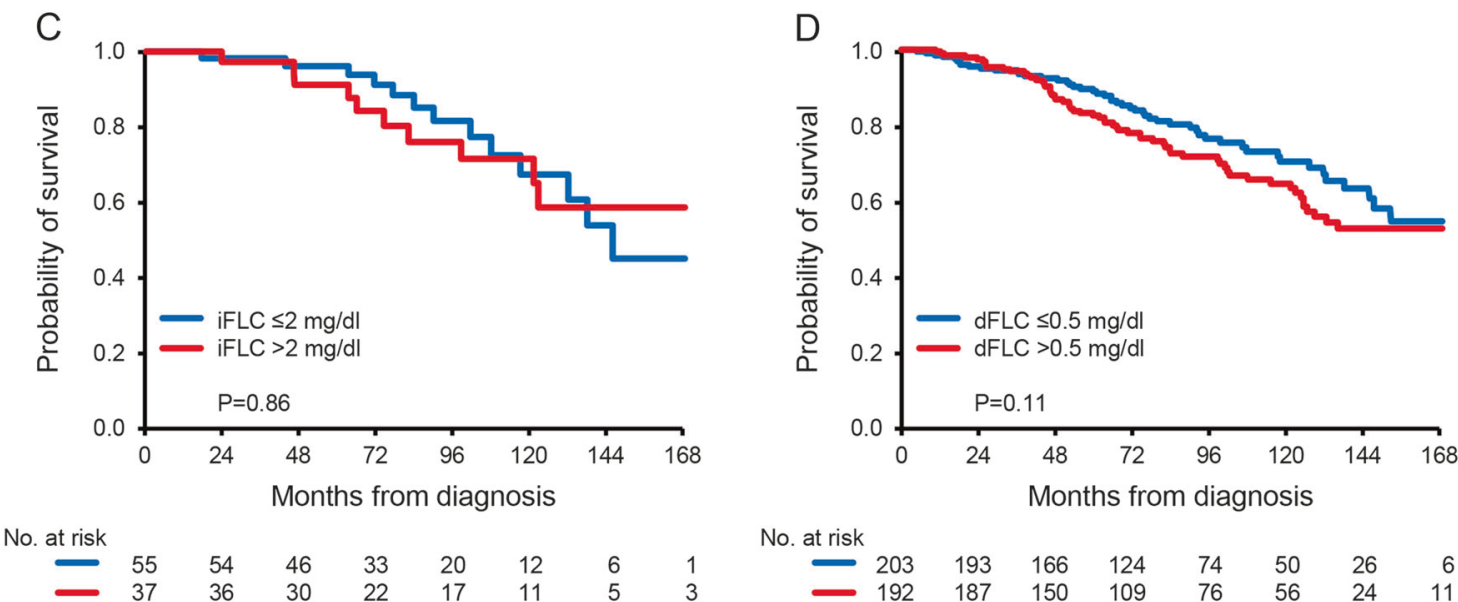

No. at risk

$\begin{array}{rrrrrrrr}203 & 193 & 166 & 124 & 74 & 50 & 26 & 6 \\ 192 & 187 & 150 & 109 & 76 & 56 & 24 & 11\end{array}$

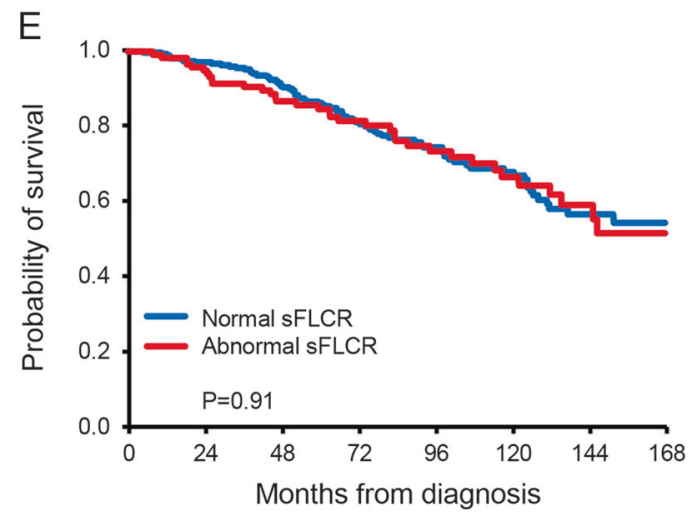

No. at risk

$\begin{array}{rrrrrrrr} \\ 277 & 268 & 226 & 167 & 100 & 73 & 33 & 10 \\ 118 & 112 & 90 & 69 & 50 & 33 & 17 & 7\end{array}$

Fig. 1 Overall survival stratified by: a iFLC level at end of therapy using the $2 \mathrm{mg} / \mathrm{dL}$ cut point in the entire study cohort. b iFLC of $2 \mathrm{mg} /$ $\mathrm{dL}$ at end of therapy in Lambda light chain amyloidosis. $\mathbf{c}$ iFLC of 2

$\mathrm{mg} / \mathrm{dL}$ at end of therapy in Kappa light chain amyloidosis. d dFLC level at end of therapy using $0.5 \mathrm{mg} / \mathrm{dL}$ cut point. e sFLC ratio normalization status at end of therapy

standard chemotherapy (median 118 vs. 53 months; $\mathrm{P}<0.001)$. An OS advantage was noted only for patients with a nadir iFLC reached $>12$ months in the standard chemotherapy subgroup (10-year OS median $56 \%$ vs. $43 \%$;

$\mathrm{P}=0.04)$, but not in the ASCT subgroup (10-year OS $86 \%$ vs. $75 \% ; \mathrm{P}=0.12$ ).

A multivariate analysis of hemPFS and OS is presented in Table 1. iFLC $\leq 2 \mathrm{mg} / \mathrm{dL}$ at end of first-line therapy and 
Table 1 Multivariate analysis of hemPFS and OS

\begin{tabular}{|c|c|c|c|c|c|c|}
\hline & \multicolumn{3}{|c|}{ HemPFS } & \multicolumn{3}{|l|}{ OS } \\
\hline & HR & $95 \% \mathrm{CI}$ & $P$ & HR & $95 \% \mathrm{CI}$ & $P$ \\
\hline Age $>65$ years & 1.4 & $1.0-1.9$ & 0.047 & 2.3 & $1.5-3.5$ & $<0.001$ \\
\hline BMPCs $>10 \%$ & 1.5 & $1.1-2.0$ & 0.009 & 1.6 & $1.05-2.4$ & $\mathbf{0 . 0 3}$ \\
\hline ASCT as primary therapy & 0.8 & $0.6-1.1$ & 0.24 & 0.6 & $0.4-0.9$ & 0.02 \\
\hline Mayo stage 2012 (III/IV vs. I/II) & 1.0 & $0.7-1.5$ & 0.9 & 1.9 & $1.1-3.1$ & 0.01 \\
\hline $\mathrm{iFLC} \leq 2 \mathrm{mg} / \mathrm{dL}$ by the end of first line therapy & 0.3 & $0.2-0.4$ & $<0.001$ & 0.4 & $0.2-0.6$ & $<0.001$ \\
\hline Nadir iFLC $>12$ months from the end of therapy & 0.3 & $0.2-0.4$ & $<0.001$ & 0.4 & $0.2-0.7$ & $<0.001$ \\
\hline
\end{tabular}

ASCT autologous stem cell transplantation, BMPCs bone marrow plasma cells, $C I$ confidence interval, $H R$ hazard ratio, $i F L C$ involved free light chain, $O S$ overall survival, HemPFS hematological progression-free survival. Boldface indicates significance at $\mathrm{P}<0.05$

time to nadir iFLC > 12 months were strong predictors for hemPFS and OS. An alternative model which includes response category had similar output (Supplementary Table 4).

We have demonstrated the importance of achieving a low iFLC at the end of first-line therapy in AL amyloidosis. Our data support that iFLC should be reduced as low as possible, preferably to $\leq 2 \mathrm{mg} / \mathrm{dL}$. This is associated with a higher organ response rate and improved hemPFS and OS. sFLCR Normalization does not predict for organ response or survival and should not be used as a measure of therapeutic efficacy. It is not always possible to determine if elevation of the iFLC is polyclonal, often secondary to significant renal impairment or perhaps infection/inflammation. In such cases, a bone marrow biopsy coupled with multiparametric flow cytometry study may be required to determine the persistence of clonal plasma cells [8,9], which would argue for further chemotherapy.

Anti-plasma cell therapies are toxic to normal B-cell and plasma cell populations. This will cause immunoparesis (including of serum free light chains) at the end of therapy with anticipated recovery over time. Therefore, it is not surprising that most patients have achieved nadir iFLC close to the end of therapy, at a median of 4 months. However, some patients achieved a nadir iFLC more than a year following therapy completion. This predicted long-term control of the amyloidogenic clone and is associated with improved outcome. Time to nadir iFLC is also independent of depth of iFLC at completion of therapy. ASCT was more likely to result in a nadir iFLC well beyond the end of therapy (median of 8 months from end of therapy), which matches our experience of longer remission achieved with ASCT.

Study limitations include its retrospective design and lack of systematic assessment of sFLC assay after completion of therapy, which may impact ability to accurately capture time of nadir iFLC. The cohort size should reduce sampling bias.
Acknowledgements The study was supported in part by the Jabbs Foundation (Birmingham, United Kingdom), the Henry J. Predolin Foundation (USA), and National Institutes of Health National Cancer Institute grant P50 CA186781.

Author contributions: EM designed the study, analyzed the data, wrote the first draft, and approved the final version of the manuscript; AD, NL, MQL, FKB, DD, SRH, PK, YLH, AF, MH, WG, TK, RW, SR, JAL, YL, RSG, SZ, and SVK. SKK performed patient management, revised the manuscript critically and approved the final version of the manuscript. RAK performed patients' follow-up, revised the manuscript critically and participated in final data analysis and approval of the final version of the manuscript; MAG performed patient management, designed the study, analyzed the data, wrote the first draft and approved the final version of the manuscript.

\section{Compliance with ethical standards}

Conflict of interest AD: Research funding (Celgene, Millennium, Pfizer, and Janssen), Travel grant (Pfizer); MQL: Research funding (Celgene); DD: Research funding (Karyopharm Therapeutics, Amgen, and Millenium Pharmaceuticals); PK: Research funding (Takeda, Celgene, and Amgen); SK: Consultancy (Celgene, Millennium, Onyx, Janssen, and BMS); and research funding (Celgene, Millennium, Novartis, Onyx AbbVie, Janssen, and BMS). MAG: Consultancy (Milleniu) and honoraria (Celgene, Millenium, Onyx, Novartis, Smith Kline, Prothena, Ionis). The remaining authors declare that they have no conflict of interest.

\section{References}

1. Dispenzieri A, Lacy MQ, Katzmann JA, Rajkumar SV, Abraham RS, Hayman SR, et al. Absolute values of immunoglobulin free light chains are prognostic in patients with primary systemic amyloidosis undergoing peripheral blood stem cell transplantation. Blood. 2006;107:3378-83.

2. Kumar S, Dispenzieri A, Katzmann JA, Larson DR, Colby CL, Lacy MQ, et al. Serum immunoglobulin free light-chain measurement in primary amyloidosis: prognostic value and correlations with clinical features. Blood. 2010;116:5126-9.

3. Lachmann HJ, Gallimore R, Gillmore JD, Carr-Smith HD, Bradwell AR, Pepys MB, et al. Outcome in systemic AL amyloidosis in relation to changes in concentration of circulating free immunoglobulin light chains following chemotherapy. $\mathrm{Br} \mathrm{J}$ Haematol. 2003;122:78-84. 
4. Sanchorawala V, Seldin DC, Magnani B, Skinner M, Wright DG. Serum free light-chain responses after high-dose intravenous melphalan and autologous stem cell transplantation for AL (primary) amyloidosis. Bone Marrow Transplant. 2005;36: 597-600.

5. Palladini G, Dispenzieri A, Gertz MA, Kumar S, Wechalekar A, Hawkins PN, et al. New criteria for response to treatment in immunoglobulin light chain amyloidosis based on free light chain measurement and cardiac biomarkers: impact on survival outcomes. J Clin Oncol. 2012;30:4541-9.

6. Tandon N, Sidana S, Dispenzieri A, Gertz MA, Lacy MQ, Dingli $\mathrm{D}$, et al. Impact of involved free light chain (FLC) levels in patients achieving normal FLC ratio after initial therapy in light chain amyloidosis (AL). Am J Hematol. 2018;93:17-22.
7. Comenzo RL, Reece D, Palladini G, Seldin D, Sanchorawala V, Landau $\mathrm{H}$, et al. Consensus guidelines for the conduct and reporting of clinical trials in systemic light-chain amyloidosis. Leukemia. 2012;26:2317-25.

8. Muchtar E, Jevremovic D, Dispenzieri A, Dingli D, Buadi FK, Lacy MQ, et al. The prognostic value of multiparametric flow cytometry in AL amyloidosis at diagnosis and at the end of firstline treatment. Blood. 2017;129:82-87.

9. Sidana S, Tandon N, Dispenzieri A, Gertz MA, Rajkumar SV, Kumar SK. The importance of bone marrow examination in patients with light chain amyloidosis achieving a complete response. Leukemia. 2018;32:1243-1246

\title{
Daratumumab-based therapy in patients with heavily-pretreated AL amyloidosis
}

\author{
Jithma P. Abeykoon ${ }^{1,2}$. Saurabh Zanwar $\mathbb{D}^{1,2} \cdot$ Angela Dispenzieri ${ }^{1,2} \cdot$ Morie A. Gertz ${ }^{1,2} \cdot$ Nelson Leung $\mathbb{D}^{1,2,3}$. \\ Taxiarchis Kourelis ${ }^{1,2} \cdot$ Wilson Gonsalves ${ }^{1,2} \cdot$ Eli Muchtar $\mathbb{D}^{1,2} \cdot$ David Dingli $^{1,2} \cdot$ Martha Q. Lacy ${ }^{1,2}$. \\ Suzanne R. Hayman ${ }^{1,2} \cdot$ Francis Buadi $^{1,2} \cdot$ Rahma Warsame $^{1,2} \cdot$ Robert A. Kyle $^{1,2} \cdot$ Vincent Rajkumar $^{1,2}$. \\ Shaji Kumar $\mathbb{D}^{1,2} \cdot$ Prashant Kapoor ${ }^{1,2}$
}

Received: 26 June 2018 / Revised: 15 August 2018 / Accepted: 21 August 2018 / Published online: 28 September 2018

(c) Springer Nature Limited 2018

\section{Introduction}

Immunoglobulin light chain amyloidosis (AL) results from extracellular deposition of light chain-composed amyloid fibrils leading to organ dysfunction. Plasma cell (PC)-

These authors contributed equally: Jithma P. Abeykoon and Saurabh Zanwar.

This research work was presented at the American Society of Clinical Oncology Annual Meeting, Chicago, June 2018.

Electronic supplementary material The online version of this article (https://doi.org/10.1038/s41375-018-0262-2) contains supplementary material, which is available to authorized users.

$\triangle$ Prashant Kapoor

kapoor.prashant@mayo.edu

1 Department of Internal Medicine, Mayo Clinic, Rochester, MN, USA

2 Division of Hematology, Mayo Clinic, Rochester, MN, USA

3 Division of Nephrology, Mayo Clinic, Rochester, MN, USA directed therapy in AL induces hematologic response (HR) and, in turn, organ response (OR), with improvement in quality-of-life (QoL) and overall-survival (OS) [1, 2]. Despite therapeutic advances, AL remains a challenging disease to treat and newer therapies are needed.

Daratumumab is an anti-CD38 monoclonal antibody, with an established role, both as monotherapy with an overall response-rate (ORR) of $30 \%$ in heavily-pretreated multiple myeloma (MM) [3] and in combination with alkylatingagents, proteasome-inhibitors and immunomodulatory-agents (ORR $>90 \%$ in both newly-diagnosed and pretreated MM patients) [3, 4]. As in MM, AL-associated PCs uniformly express CD38, the target glycoprotein for daratumumab [5]. Although daratumumab has been studied in relapsed and/or refractory (RR) AL, with impressive preliminary results [6-9], only a single study evaluating daratumumab monotherapy (DMT) has been published [5]. Furthermore, the efficacy of daratumumab combination therapy (DCT) in AL is unknown. We examined the impact of daratumumab-based therapy (DBT) in RRAL.

Following approval by the Institutional Review Board, medical records of all patients with $\mathrm{AL}$, consecutively seen 
Table 1a Baseline characteristics at initiation of daratumumab-based therapy

\begin{tabular}{|c|c|c|c|}
\hline Parameter & $\begin{array}{l}\text { Total cohort, } n= \\
44\end{array}$ & DMT, $n=22$ & DCT, $n=22$ \\
\hline \multicolumn{4}{|l|}{ Revised 2012 Mayo stage, $n(\%)$} \\
\hline Stage I & $3(7.5)$ & $2(10)$ & $1(5)$ \\
\hline Stage II & $19(47.5)$ & $7(35)$ & $12(60)$ \\
\hline Stage III & $12(30)$ & $7(35)$ & $5(25)$ \\
\hline Stage IV & $6(15)$ & $4(20)$ & $2(10)$ \\
\hline \multicolumn{4}{|l|}{ Mayo stage $2004, n(\%)$} \\
\hline Stage I & $11(27.5)$ & $5(25)$ & $6(30)$ \\
\hline Stage II & $17(42.5)$ & $5(25)$ & $12(60)$ \\
\hline Stage III & $12(30)$ & $10(50)$ & $2(10)$ \\
\hline $\begin{array}{l}\text { a. Stage III disease with SBP }>100 \mathrm{mmHg} \text { and NT- } \\
\text { proBNP }<8500 \mathrm{ng} / \mathrm{L}, n\end{array}$ & 7 & 6 & 1 \\
\hline $\begin{array}{l}\text { b. Stage III disease with } \mathrm{SBP}<100 \mathrm{mmHg} \text { or NT- } \\
\text { proBNP }>8500 \mathrm{ng} / \mathrm{L}, n\end{array}$ & 4 & 4 & 0 \\
\hline $\begin{array}{l}\text { c. Stage III disease with } \mathrm{SBP}<100 \mathrm{mmHg} \text { and NT- } \\
\text { proBNP }>8500 \mathrm{ng} / \mathrm{L}, n\end{array}$ & 1 & 0 & 1 \\
\hline Hemoglobin, median (range), g/dL & $12(8.2-14.6)$ & $\begin{array}{l}11.7 \\
(8.2-14.1)\end{array}$ & $12.3(8.3-14.6)$ \\
\hline Serum creatinine, median (range), $\mathrm{mg} / \mathrm{dL}^{\mathrm{a}}$ & $1.25(0.6-6.6)$ & $1.2(0.6-4.1)$ & $1.25(0.6-6.6)$ \\
\hline Serum albumin, median (range), $\mathrm{g} / \mathrm{dL}^{\mathrm{a}}$ & $3.2(1.8-4.3)$ & $3.2(1.8-4.3)$ & $3.2(2-4)$ \\
\hline NT-proBNP, median (range), $\mathrm{pg} / \mathrm{mL}^{\mathrm{a}}$ & $908(24-18913)$ & $\begin{array}{l}908 \\
(68-18363)\end{array}$ & $\begin{array}{l}1001 \\
(24-18913)\end{array}$ \\
\hline Troponin- $\mathrm{T}$, median (range), $\mathrm{ng} / \mathrm{mL}^{\mathrm{a}}$ & $0.01(0-0.41)$ & $0.03(0-0.41)$ & $0(0-0.23)$ \\
\hline $\begin{array}{l}\text { Intraventricular septal thickness, median (range), } \\
\mathrm{mm}^{\mathrm{a}}\end{array}$ & $14(8-21)$ & $16(13-21)$ & $12.5(8-17)$ \\
\hline NYHA class (range) ${ }^{\mathrm{a}}$ & $1(0-3)$ & $1(0-3)$ & $1(0-2)$ \\
\hline Alkaline phosphatase, median (range), $\mathrm{U} / \mathrm{L}^{\mathrm{a}}$ & $68(18-994)$ & $78(18-994)$ & $67(23-245)$ \\
\hline Protein in urine, median (range), $\mathrm{g} / 24 \mathrm{~h}^{\mathrm{a}}$ & $0.8(0.03-13)$ & $0.9(0.14-13)$ & $0.8(0.03-7.9)$ \\
\hline $\begin{array}{l}\text { Serum Kappa FLC in Kappa restricted disease, } \\
\text { median (range), mg/dL }\end{array}$ & $13.8(1.3-71.4)$ & $14(1.7-71.4)$ & $13.6(1.3-53)$ \\
\hline $\begin{array}{l}\text { Serum Lambda FLC in Lambda restricted disease, } \\
\text { median (range), } \mathrm{mg} / \mathrm{dL}\end{array}$ & $10(2.7-92.3)$ & $7.0(2.7-62.1)$ & $12.7(2.7-92.3)$ \\
\hline IgA, median (range), $\mathrm{mg} / \mathrm{dL}$ & $76(4-398)$ & $79(4-398)$ & $53(5-189)$ \\
\hline $\mathrm{IgG}$, median (range), $\mathrm{mg} / \mathrm{dL}$ & $575.5(68-1680)$ & $675(205-956)$ & $544(68-1680)$ \\
\hline IgM, median (range), $\mathrm{mg} / \mathrm{dL}$ & $37(5-877)$ & $29(16-113)$ & $39(5-877)$ \\
\hline Kappa FLC involved, $n(\%)$ & $14(32)$ & $7(32)$ & $7(33)$ \\
\hline $\mathrm{dFLC}$, median (range), $\mathrm{mg} / \mathrm{dL}$ & $8.2(0.6-301)$ & $7(1.0-70)$ & $10.1(0.6-301)$ \\
\hline Marrow clonal plasma cell, median \% (range) & $5(1-15)$ & $5(1-15)$ & $4(1-15)$ \\
\hline \multicolumn{4}{|l|}{ Organ involvement, $n(\%)$} \\
\hline Heart & $27(61)$ & $14(64)$ & $13(59)$ \\
\hline Kidney & $22(50)$ & $11(50)$ & $11(50)$ \\
\hline Liver & $3(7)$ & $2(9)$ & $1(5)$ \\
\hline
\end{tabular}

DMT Daratumumab monotherapy, DCT Daratumumab combination therapy, NYHA New York Heart Association, NT-pro BNP N-terminal proBeta natriuretic peptide, FLC free light chain, $d F L C$ difference in serum free light chains, SBP systolic blood pressure a assessed for the entire cohort, $n=44$ and not only for patients with respective organ involvement

at Mayo Clinic, Rochester, between 01/01/2015 and 02/15/ 2018 were reviewed and the patients with $\mathrm{AL}$ who were treated with daratumumab (as mono-therapy or combination-therapy), at the currently approved dose and frequency for MM, in RR setting were assessed. The patients with coexisting symptomatic MM were excluded. Response, including progression, was assessed by the 2012 criteria $[2,10]$ Measurable disease was defined as a difference in 
Table 1b Exposure and refractoriness to specific agents in patients who received DMT or DCT

\begin{tabular}{lllll}
\hline $\begin{array}{l}\text { Treatment before } \\
\text { DMT/DCT }\end{array}$ & $\begin{array}{l}\text { Patients } \\
\text { exposed }(\%)\end{array}$ & \% refractory & $\begin{array}{l}\text { Refractory patients } \\
\text { receiving DMT }(n)\end{array}$ & $\begin{array}{l}\text { Refractory patients } \\
\text { receiving DCT }(n)\end{array}$ \\
\hline Bortezomib & 91 & 45 & 10 & 10 \\
Lenalidomide & 57 & 29 & 5 & 3 \\
Carfilzomib & 16 & 7 & 0 & 3 \\
Pomalidomide & 20 & 7 & 0 & 2 \\
$\begin{array}{l}\text { Ixazomib } \\
\text { Melphalan (outside }\end{array}$ & 11 & 7 & 3 & 0 \\
$\begin{array}{l}\text { of ASCT) } \\
\text { High-dose Melphalan }\end{array}$ & 23 & 7 & & - \\
(ASCT) & 52 & 0 & - & 6 \\
Cyclophosphamide & 75 & 32 & & \\
\hline
\end{tabular}

DMT Daratumumab monotherapy, DCT Daratumumab combination therapy, ASCT autologous stem cell transplant

serum free light chains (dFLC) of at least $5 \mathrm{mg} / \mathrm{dL}$ at DBT initiation; patients with $\mathrm{dFLC}<5 \mathrm{mg} / \mathrm{dL}$ at DBT initiation were analyzed seperately [11]. Refractoriness to prior therapy was defined as disease progression on therapy or within 2 months of discontinuing treatment. Progressionfree survival (PFS) was defined as the interval from the first dose of DBT to disease progression, or death from any cause, whichever occurred first. Event-free survival (EFS) was defined as the interval from initiation of DBT till discontinuation of DBT due to toxicity, progression or death due to any cause. Duration of response was defined as interval from the first documentation of at least partial response (PR) to progression or death due to disease, with patients dying from causes other than AL being censored. All time-to-event analyses were performed from daratumumab initiation using the Kaplan-Meier method on JMP 13.0 software.

Forty-four patients received DBT; median age at DBT initiation was 64 years (range: 46-82). Twenty-four (54\%) patients were male. The median time from diagnosis to DBT initiation was 4.0 years (range: $0.4-17$ ) and median follow-up from DBT initiation was 10.2 months (95\% CI: 8.0-13.1). The Mayo 2004 Stage with European modification [12], Revised-Mayo 2012 Stage, laboratory parameters and the type of organ(s) involved are outlined in Table $1 \mathrm{a}$. The median number of prior therapies was 3 (range: $1-8$ ) and $11 \%$ of patients had received more than four lines of therapy previously. Prior therapy-related data are depicted in Table 1b. Twenty-two (50\%) patients received DMT and $22(50 \%)$ received DCT. The decision to commence DMT or DCT was per the clinician's preference. The median number of cycles of DBT was 8.0 (range: 2-24). Daratumumab, pomalidomide and dexamethasone (DPD, 36\%), daratumumab, lenalidomide and dexamethasone (DRD, $32 \%$ ) and daratumumab, bortezomib and dexamethasone (DVD, 18\%) were the most commonly used DCTs. The main treatment-emergent adverse effects (AEs) are
Table 1c Treatment-emergent adverse effects (National Cancer Institute Common Toxicity Criteria v4.03)

\begin{tabular}{llll}
\hline Adverse effect & $\begin{array}{l}\text { \% of } \\
\text { patients }\end{array}$ & $\begin{array}{l}\text { Patients receiving } \\
\text { DMT }(n)\end{array}$ & $\begin{array}{l}\text { Patients } \\
\text { receiving DCT } \\
(n)\end{array}$ \\
\hline Anemia grade & & & \\
1 & 41 & 8 & 9 \\
2 & 19 & 3 & 5 \\
3 & 2 & 1 & 0 \\
Thrombocytopenia grade & & & \\
1 & 38 & 5 & 11 \\
Neutropenia grade & & & \\
1 & 10 & 1 & 3 \\
2 & 12 & 1 & 4 \\
3 & 7 & 0 & 2 \\
Infusion reaction & 22 & 7 & 4 \\
Neuropathy & 17 & 3 & 2 \\
Infection & 7 & 1 & 3 \\
Diarrhea & 13 & 0 & 5 \\
Fatigue & 24 & 5 & 2 \\
Dyspnea & 7 & 1 & 1 \\
VTE & 5 & 1 & 1 \\
CHF & 2 & 0 & 0 \\
Hypertension & 2 & 1 & 2 \\
Increase in baseline & 9 & 2 & \\
creatinine & & & \\
\hline
\end{tabular}

DMT: Daratumumab monotherapy; DCT: Daratumumab combination therapy; $C H F$ : congestive heart failure; VTE: venous thromboembolism

highlighted in Table 1c. Twenty-two (50\%) patients (11 each in DMT and DCT groups) were refractory to their last line of treatment before initiation of DBT. Table 2 outlines the depth of HR in patients who had $\geq 5 \mathrm{mg} / \mathrm{dL}$ dFLC ( $n=$ 30) at DBT initiation. Among the remaining 14 patients with $\mathrm{dFLC}<5 \mathrm{mg} / \mathrm{dL}$ at DBT initiation (8 in DMT and 6 in DCT group), $43 \%(n=6)$ achieved complete response and the rest also continued to remain progression-free at last follow-up. Of the 14 patients, 6 had dFLC between $2 \mathrm{mg} / \mathrm{dL}$ and $5 \mathrm{mg} / \mathrm{dL}$ at DBT initiation, and 4 of these 6 patients 
Table 2 Hematologic response and outcomes

\begin{tabular}{|c|c|c|c|}
\hline & $\begin{array}{l}\text { Entire cohort, } \\
n=44^{\mathrm{a}}\end{array}$ & $\begin{array}{l}\text { DMT, } \\
n=22^{\mathrm{b}}\end{array}$ & $\begin{array}{l}\text { DCT, } \\
n=22^{\mathrm{c}, \mathrm{d}, \mathrm{e}}\end{array}$ \\
\hline Prior lines of therapy, $n$ (range) & $3(1-8)$ & $3(1-5)$ & $3(1-8)$ \\
\hline $\mathrm{ORR}^{\mathrm{a}}, n(\%)$ & $25(83)$ & $11(78)$ & $14(88)$ \\
\hline $\mathrm{CR}^{\mathrm{a}}, n(\%)$ & $5(17)$ & $2(14)$ & $3(19)$ \\
\hline $\mathrm{VGPR}^{\mathrm{a}}, n(\%)$ & $19(63)$ & $9(64)$ & $10(63)$ \\
\hline $\mathrm{PR}^{\mathrm{a}}, n(\%)$ & $1(3)$ & 0 & $1(6)$ \\
\hline $\begin{array}{l}\text { Time to } 1^{\text {st }} / \text { best response, months ( } 95 \% \\
\text { CI) }\end{array}$ & $2.2(1.7-4.8) / 5.7(3.4-7.7)$ & $\begin{array}{l}2.6(1.7-6.2) / 6.2 \\
(2.2-8.9)\end{array}$ & $\begin{array}{l}1.9(0.9-3.4) / 5.7 \\
(2.3-12.2)\end{array}$ \\
\hline Median follow-up, months $(95 \% \mathrm{CI})$ & $10.2(8.0-13.1)$ & $7.7(5.6-10.4)$ & $13.1(9.1-17.8)$ \\
\hline PFS, months (95\% CI) & NR (15.5-NR) & NR (13.2-NR) & NR (15.5-NR) \\
\hline PFS for 6 and 10 months ( $\%$ of patients) & $97 \%$ and $89 \%$ & $100 \%, 100 \%$ & $100 \%, 83 \%$ \\
\hline OS, months $(95 \% \mathrm{CI})$ & NR (NR-NR) & NR (13-NR) & NR (15.5-NR) \\
\hline OS for 10 months (\% of patients) & $94 \%$ & $100 \%$ & $89 \%$ \\
\hline EFS, months $(95 \% \mathrm{CI})$ & 15.5 (12-NR) & $13.2(8.1-15.0)$ & NR (11.3-NR) \\
\hline EFS for 6 and 10 months (\% of patients) & $86 \%$ and $76 \%$ & $80 \%$ and $56 \%$ & $90 \%$ and $81 \%$ \\
\hline
\end{tabular}

$E F S$ event-free-survival, $O R R$ overall response rate, $C R$ complete response, $V G P R$ very good partial response, $P R$ partial response, $P F S$ progression free survival, $O S$ overall survival, $C I$ confidence interval, $N R$ not reached

a30 patients were evaluable for hematologic response

${ }^{\mathrm{b}} 14$ evaluable for hematologic response

${ }^{\mathrm{c}} 16$ evaluable for hematologic response

${ }^{\mathrm{d}}$ Two patients on DCT were first treated with DMT; in one patient who ultimately received DCT, daratumumab monotherapy was initially administered to assess initial tolerance to this agent. In the other patient DCT was administered due to progressive disease on DMT.

${ }^{e}$ Daratumumab was added to ongoing regimen due to inadequate response in 3 patients ( 2 non-responders and 1 with partial response as best response), resulting in complete response in two patients and very good partial response in one patient, subsequently.

attained $\mathrm{dLFC}<1 \mathrm{mg} / \mathrm{dL}$ following initiation of DBT. At last follow-up, 10 patients had discontinued DBT and 4 had died while on DBT. The reasons for discontinuation of DBT included progression $(n=1)$, fatigue $(n=2)$, lack of response $(n=1)$, sigmoid perforation $(n=1)$, worsening neuropathic pain $(n=1)$, autologous-stem-cell transplant after achieving VGPR $(n=1)$, concern of cardiac rejection after cardiac transplantation for AL $(n=1)$, myelodysplastic syndrome $(n=1)$ and patient opting for palliative care only $(n=1)$. Three patients received subsequent line of treatment after DBT discontinuation and the median timeto-next therapy was 1.8 months (95\% CI: $1.0-10)$

The median time-to-first HR for the entire cohort was 2.2 months (95\% CI: 1.7-4.8); 2.6 months (95\% CI: $1.7-$ 6.2) with DMT and 1.9 months (95\% CI: 0.9-3.4) for patients treated with DCT and median duration of HR was NR in either group. Two (4.5\%) patients had progressive disease. Additional response assessment data, including frequency of assessment and HR after the first and second cycle of DBT are outlined in the Supplementary Material. Among patients with cardiac (61\%), kidney (50\%) and liver (7\%) involvement, the respective OR rates were $44 \%$ (43\% with DMT and 46\% with DCT), $27 \%$ (18\% with DMT and 36\% with DCT) and $0 \%$ at last follow-up. The median time-to-best cardiac response was 13.5 months (95\% CI: 7.5-NR) for the entire cohort; 8.3 months $(95 \%$ CI: 6.2-NR) in patients treated with DMT and 14.6 months (95\% CI: 4.6-NR) in patients treated with DCT. Median time-to-best renal response was NR (95\% CI: 5 months-NR) for the entire cohort; NR (95\% CI: 2.2 months-NR) in patients treated with DMT and NR (95\% CI: 3.0 monthsNR) in patients treated with DCT. Table 2 demonstrates other outcome data.

Our study underscores the safety and efficacy of both DMT and DCT in RRAL. To our knowledge, this is the first study to report outcomes with DCT in heavily-pretreated AL. An impressive HR of 83\% (88\% with DCT, 78\% with DMT) comparable to those in the previously published reports $[5,6]$ predominantly VGPR or deeper, is remarkable considering our multiply RR patient-population. Two studies, currently in abstract form, reported HR of $63 \%$ after 3 months follow-up [7] and 50\% after one dose of daratumumab, respectively [8]. In some studies, patients with coexisting MM or dFLC $<5$ $\mathrm{mg} / \mathrm{dL}$ have been included for HR assessment [7, 10], making response-assessment inaccurate.

Our results are particularly encouraging compared to historically observed HR rates of $50-75 \%$ with conventional chemotherapies [13]. Furthermore, traditional chemotherapeutic agents are commonly associated with substantial toxicities, with more than one-third of patients experiencing zgrade 3 AEs [13]. Hematologic-response rates of $40-80 \%$ [13] with bortezomib, $40-70 \%$ with lenalidomide-based regimens [14] and 48-68\% with pomalidomide-dexamethasone have been documented in RRAL [15]. 
Although the baseline characteristics and organ involvement for patients on DMT and DCT appeared comparable, in the absence of randomization, a formal statistical comparison of the 2 groups was avoided. Importantly, the high efficacy of daratumumab was coupled with a favorable safety profile in both groups, with less than $10 \%$ of patients exhibiting grade 3 anemia and neutropenia and none experiencing severe thrombocytopenia. One patient with gastrointestinal involvement developed bowel perforation on DMT while another had a myelodysplastic syndrome, presumed to be related to prior exposure to melphalan and cyclophosphamide. Although, a phase II study $(n=8)$ reported no significant infusion-related reactions (IRR), possibly due to elaborate prophylaxis [9], other recent studies have reported 33-60\% IRR [5, 8]. Our study, showed comparable rates $(22 \%)$, which were also similar to our experience in MM [3]. However, retrospective data collection may have underestimated IRR.

For accurate HR assessment, we excluded patients with concomitant MM. Additionally, larger patient-population in our study, and a longer follow-up may have improved the validity and accuracy of the OR rates compared to those reported in prior studies [6, 7]. However, the limitations inherent to our retrospective study design persist, including underestimation of treatment-emergent AEs and absence of QoL data. Further, given that this cohort had received median three prior lines of therapy, a selection bias with inclusion of less fit patients may exist. We recognize that the use of immunomodulatory drugs (IMiDs) can increase NT-proBNP levels and can interfere with adequate cardiac response assessment [1]. None of the patients on an IMiDbased DCT $(n=9)$ in our study had a cardiac progression per the 2012 Consensus criteria, thereby ruling out any false progression events. Since our study incorporated patients treated outside of clinical trial setting, response assessment was not at regular intervals which may have adversely impacted the assessment of time-to-first HR and time-tobest HR.

In summary, daratumumab, both as monotherapy and in combination, appears safe and highly effective in heavily pretreated AL-amyloidosis. Rapid achievement of HR with daratumumab-based therapy, particularly with DCT, is important. Notably, these results appear to be far superior to those observed with DMT and DCT in MM, highlighting the value of targeting CD38 in AL, a disease typically with substantially lower level of clonal plasmacytosis. Additionally the favorable toxicity profile makes it a particularly valuable agent for $\mathrm{AL}$ associated with major organ, such as cardiac and/or renal dysfunction. Early results for cardiac and renal responses and outcome measures are promising, but extended follow-up will shed more light on these endpoints.
Acknowledgements We acknowledge all the patients, their families and the clinicians who were involved in the patients' care. This research work has received ASCO 2018 Merit Award.

Author contributions JPA, SZ and PK designed the study. JPA, SZ and PK collected analyzed and interpreted the data and wrote the first draft of the manuscript. AD, MAG, NL, TK, WG, EM, DD, MQL, SRH, FB, RW, RAK, VR and SK interpreted the data, critically revised the manuscript and all authors approved the final version.

\section{Compliance with ethical standards}

Conflict of interest Dr. Gertz has received funding from Amgen, Prothena, Annexon, Appellis, Johnson and Johnson and Celgene. Dr. Dispenzieri has received research funding from Jannsen, Takeda, Celgene, Pfizer, Alnylam, Prothena, and GSK and serves on the advisory board for Takeda and Intellia. Dr. Kumar received research grants for clinical trials from Celgene, Takeda, Janssen, BMS, Sanofi, KITE, Merck, Abbvie, Medimmune, Novartis, Roche-Genentech, Amgen. Dr. Leung serves on the advisory board for Takeda and Prothena. Dr. Kapoor is principal investigator on studies for which Mayo Clinic receives funding from Takeda, Sanofi, and Amgen. The remaining authors declare no conflicts of interest.

\section{References}

1. Warsame R, Kumar SK, Gertz MA, Lacy MQ, Buadi FK, Hayman SR, et al. Hematology patient reported symptom screen to assess quality of life for AL amyloidosis. Am J Hematol. 2017;92:435-40.

2. Palladini G, Dispenzieri A, Gertz MA, Kumar S, Wechalekar A, Hawkins PN, et al. New criteria for response to treatment in immunoglobulin light chain amyloidosis based on free light chain measurement and cardiac biomarkers: impact on survival outcomes. J Clin Oncol. 2012;30:4541-9.

3. Lakshman A, Abeykoon JP, Kumar SK, Rajkumar SV, Dingli D, Buadi FK, et al. Efficacy of daratumumab-based therapies in patients with relapsed, refractory multiple myeloma treated outside of clinical trials. Am J Hematol. 2017;92:1146-55.

4. Dimopoulos MA, Oriol A, Nahi H, San-Miguel J, Bahlis NJ, Usmani SZ, et al. Daratumumab, lenalidomide, and dexamethasone for multiple myeloma. N Engl J Med. 2016;375:1319-31.

5. Kaufman GP, Schrier SL, Lafayette RA, Arai S, Witteles RM, Liedtke M. Daratumumab yields rapid and deep hematologic responses in patients with heavily pretreated AL amyloidosis. Blood. 2017;130:900-2.

6. Khouri J, Bicky T, Reu FJ, Samaras CJ, Liu HD, Karam MA, et al. Daratumumab is safe and highly effective in AL amyloidosis. Blood. 2017;130(Suppl 1):1819-1819.

7. Kimmich C, Schönland S, Ziehl R, Ho AD, Dittrich T, MüllerTidow C, et al. Daratumumab monotherapy in thirty-two heavily pre-treated patients with advanced systemic light-chain amyloidosis. Blood. 2017;130(Suppl 1):1837-1837.

8. Roussel M, Stoppa A-M, Perrot A, Karlin L, Arnulf B, Macro M, et al. A prospective phase II of daratumumab in previously-treated systemic light-chain (AL) amyloidosis. Blood. 2017;130(Suppl 1):508-508.

9. Sanchorawala V, Sarosiek S, Sloan JM, Brauneis D, Migre ME, Mistark M, et al. Safety and tolerability of daratumumab in patients with relapsed light chain (AL) amyloidosis: preliminary results of a phase II study. Blood. 2017;130(Suppl 1):507-507.

10. Comenzo RL, Reece D, Palladini G, Seldin D, Sanchorawala V, Landau $\mathrm{H}$, et al. Consensus guidelines for the conduct and 
reporting of clinical trials in systemic light-chain amyloidosis. Leukemia. 2012;26:2317-25.

11. Sidana S, Tandon N, Dispenzieri A, Gertz MA, Buadi FK, Lacy $\mathrm{MQ}$, et al. Clinical presentation and outcomes in light chain amyloidosis patients with non-evaluable serum free light chains. Leukemia. 2018;32:729-35.

12. Wechalekar AD, Schonland SO, Kastritis E, Gillmore JD, Dimopoulos MA, Lane T, et al. A European collaborative study of treatment outcomes in 346 patients with cardiac stage III AL amyloidosis. Blood. 2013;121:3420-7.
13. Mahmood S, Palladini G, Sanchorawala V, Wechalekar A. Update on treatment of light chain amyloidosis. Haematologica. 2014;99: 209-21.

14. Sanchorawala V, Wright DG, Rosenzweig M, Finn KT, Fennessey $\mathrm{S}$, Zeldis JB, et al. Lenalidomide and dexamethasone in the treatment of $\mathrm{AL}$ amyloidosis: results of a phase 2 trial. Blood. 2007;109:492-6.

15. Palladini G, Milani P, Foli A, Basset M, Russo F, Perlini S, et al. A phase 2 trial of pomalidomide and dexamethasone rescue treatment in patients with AL amyloidosis. Blood. 2017;129:2120-3.

Leukemia (2019) 33:536-541

https://doi.org/10.1038/s41375-018-0267-x

Myelodysplastic syndrome

\title{
Activation of NF-KB driven inflammatory programs in mesenchymal elements attenuates hematopoiesis in low-risk myelodysplastic syndromes
}

\author{
Zhen Ping $^{1} \cdot$ Si Chen ${ }^{1} \cdot$ Sjoerd J. F. Hermans ${ }^{1} \cdot$ Keane J. G. Kenswil ${ }^{1} \cdot$ Jacqueline Feyen $^{1} \cdot$ Claire van Dijk $^{1} \cdot$ \\ Eric M. J. Bindels ${ }^{1}$ - Athina M. Mylona ${ }^{1} \cdot$ Niken M. Adisty $^{1} \cdot$ Remco M. Hoogenboezem $^{1} \cdot$ Mathijs A. Sanders ${ }^{1}$. \\ Eline M. P. Cremers ${ }^{2}$ - Dicky J. Lindenbergh-Kortleve ${ }^{3}$. Janneke N. Samsom ${ }^{3}$ - Arjan A. van de Loosdrecht ${ }^{2}$. \\ Marc H. G. P. Raaijmakers ${ }^{1}$
}

Received: 22 March 2018 / Revised: 23 July 2018 / Accepted: 28 August 2018 / Published online: 12 October 2018

(c) The Author(s) 2018. This article is published with open access

\section{Highlights}

- Activation of NF- $\mathrm{KB}$ signaling in mesenchymal cells is common in LR-MDS.

- Activation of NF- $\mathrm{KB}$ in mesenchymal cells leads to transcriptional overexpression of inflammatory factors including negative regulators of hematopoiesis.

- Activation of NF- $\mathrm{KB}$ attenuates HSPC numbers and function ex vivo.

Myelodysplastic syndromes (MDS) are clonal disorders characterized by ineffective hematopoiesis and the propensity for leukemic transformation. Cumulating evidence has challenged the traditional view that MDS is exclusively driven by hematopoietic cell intrinsic factors. Mesenchymal

These authors contributed equally: Zhen Ping, Si Chen

Electronic supplementary material The online version of this article (https://doi.org/10.1038/s41375-018-0267-x) contains supplementary material, which is available to authorized users.

Marc H. G. P. Raaijmakers

m.h.g.raaijmakers@erasmusmc.nl

1 Department of Hematology, Erasmus MC Cancer Institute, Rotterdam, The Netherlands cells in the bone marrow (BM) microenvironment have emerged as key players in disease pathogenesis, as either initiating or contributing factors [1-4]. We have earlier demonstrated that the transcriptional landscape of highly purified mesenchymal elements from human low-risk MDS (LR-MDS) is distinct from normal mesenchymal cells and characterized by cellular stress and the upregulation of inflammatory molecules with known inhibitory effects on normal hematopoiesis [4]. Specifically, mesenchymal overexpression of the alarmins S100A8/9 was shown to drive genotoxic stress in hematopoietic stem/progenitor cells (HSPCs) and is related to leukemic evolution in a subset of LR-MDS patients [3]. An important question emerging from these findings is the nature of the upstream drivers of cellular stress and inflammatory programs in LRMDS mesenchyme. Here, we show that activation of NF- $\mathrm{kB}$ in mesenchymal cells is common in LR-MDS, driving transcriptional activation of inflammatory programs and attenuating HSPC function.

Department of Hematology, VU University Medical Center, Amsterdam, The Netherlands

3 Laboratory of Pediatrics, Division of Gastroenterology and Nutrition, Erasmus University Medical Center, Rotterdam, The Netherlands 
A HINATA_NF-KB_TARGETS_FIBROBLAST_UP

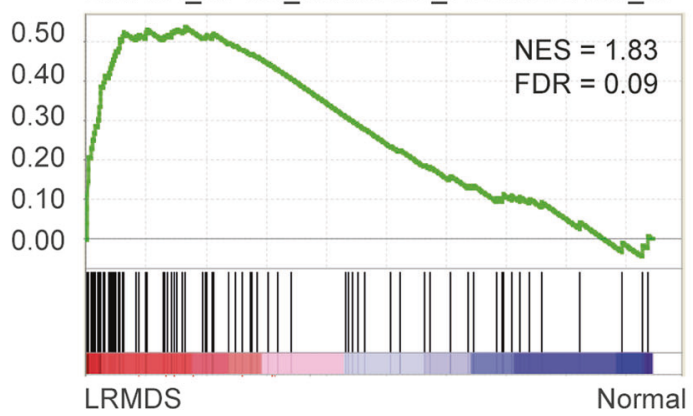

TIAN_TNF_SIGNALING VIA NF-KB

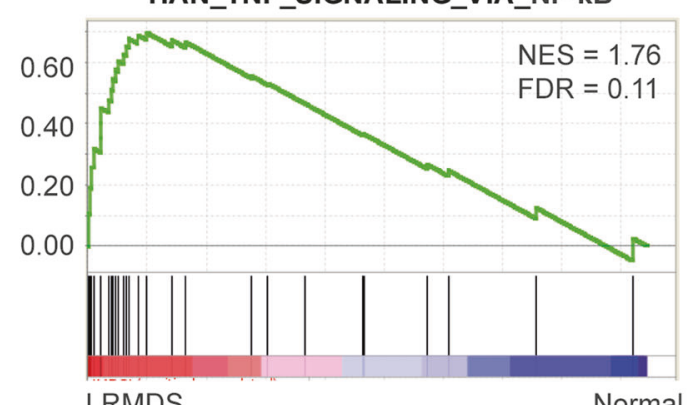

LRMDS

Normal

B

Cellular process

NF-KB Signaling
Data Sets
Enrichment Size NES FDR q-Val
HINATA_NF-KB_TARGETS_FIBROBLAST_UP TIAN_TNF_SIGNALING_VIA_NF-KB HINATA_NF-KB_TARGETS_KERATINOCYTE_UP RASHI_NF-KB_TARGETS HANSON_HRAS_SIGNALING_VIA_NF-KB SCHOEN_NF-KB_SIGNALING

$\begin{array}{llll}\text { MDS } & 84 & 1.83 & 0.09 \\ \text { MDS } & 28 & 1.76 & 0.11 \\ \text { MDS } & 91 & 1.76 & 0.13 \\ \text { MDS } & 19 & 1.67 & 0.15 \\ \text { MDS } & 22 & 1.66 & 0.16 \\ \text { MDS } & 34 & 1.63 & 0.19\end{array}$

C

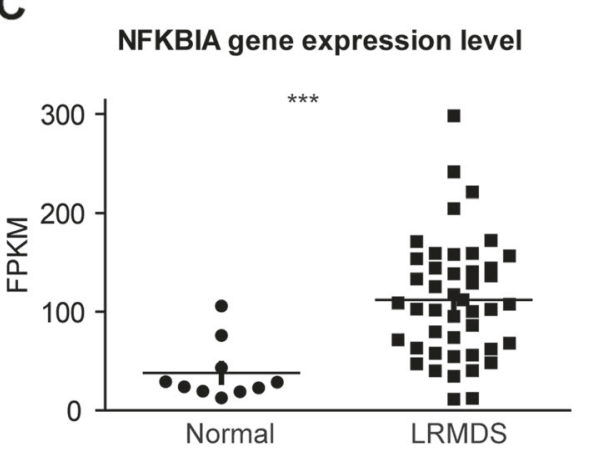

E
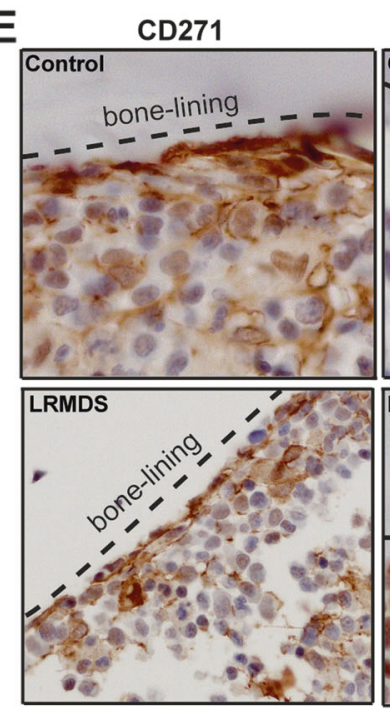

D

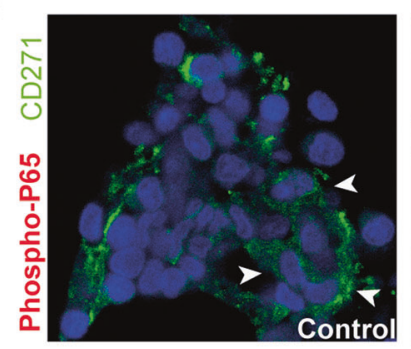

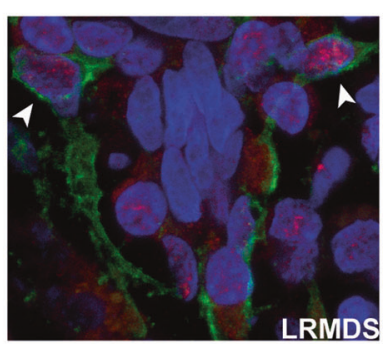
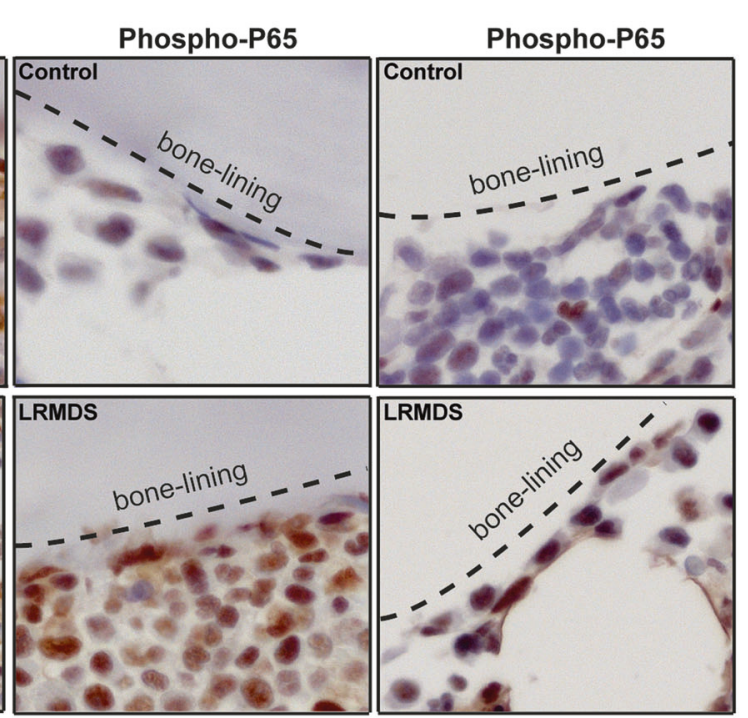

$\mathbf{F}$

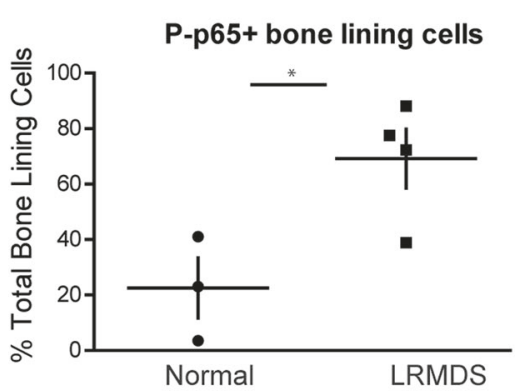

We earlier reported on the elucidation of the transcriptome of highly purified mesenchymal cells isolated from LR-MDS patients $(n=45)$ by massive parallel RNA sequencing [3], suggesting inflammation in these mesenchymal elements. In order to identify candidate master regulatory pathways upstream of inflammatory programs in LR-MDS, we performed Gene Set Enrichment Analysis (GSEA) comparing the transcriptomes of these 45 
Fig. 1 Activation of NF-кB-mediated signaling in LR-MDS mesenchymal cells. a Representative GSEA plots demonstrating activation of NF- $\mathrm{KB}$ signaling in mesenchymal cells from LR- MDS. b Summary of gene sets implicating NF- $\mathrm{KB}$ activation in mesenchymal cells from 45 LR-MDS patients. Gene set size, NES, and FDR value of each gene set are as listed. GSEA gene sets enrichment analysis, NES normalized enrichment score, FDR false discovery rate. c Gene expression level (in FPKM) of NFKBIA in normal and LR-MDS samples. d Representative images showing immunofluorescence staining of CD271 and phospho-p65 in both age-matched control (left panel) and LR-MDS patient (right panel) bone marrow slides confirming activation of NF- $\mathrm{KB}$ in mesenchymal cells. The white arrow indicates the absence or presence of nuclear phospho-p65 signal (red) in $\mathrm{CD} 271^{+}$(green) mesenchymal cells. The nuclei were counterstained with DAPI. e Representative photomicrographs of the distribution of $\mathrm{CD} 271^{+}$mesenchymal cells (left panels). These cells are enriched at the endosteal surface (marked by bone-lining area) and have a spindleshaped morphology. Representative immuno-histochemical analysis of phospho-p65 (middle and right panels) in age-matched controls (top) and LR-MDS (bottom) patients, demonstrating NF- $\mathrm{BB}$ activation in spindle-shaped endosteal cells in LR-MDS. f The percentage of phospho-p65+ bone-lining cells as a fraction of the total bone-lining cells in LR-MDS $(n=4)$ compared to age-matched controls $(n=3)$. $* * * P<.001, * P<.05$. FPKM fragments per kilobase of exon per million fragments mapped; NFKBIA NF-kappa-B inhibitor alpha

patients to mesenchymal cells purified from healthy controls $(n=10)$ [4]. In total, 120 gene signatures were significantly enriched in the LR-MDS mesenchyme, while 8 signatures were enriched in normal mesenchymal cells. Among the signatures upregulated in LR-MDS patients was a remarkable abundance of signatures related to the activation of the nuclear factor-kappa B (NF- $\mathrm{BB})$ family of transcription factors (Fig. 1a, b). To corroborate the notion of activation of this pathway and provide better insight into the heterogeneity within the population, we assessed the expression levels of NF- $\kappa \mathrm{B}$ inhibitor NFKBIA (also known as $I \kappa B-a$ ), which forms an autoregulatory loop with activated NF- $\mathrm{kB}$ transcription factors and therefore directly reflects activation of NF- $\mathrm{KB}$ signaling [5, 6]. Overexpression of NFKBIA was found in the majority of patients, suggesting that mesenchymal NF- $\mathrm{kB}$ activation is a common feature in LRMDS (Fig. 1c). To confirm the functional activation of NF$\kappa \mathrm{B}$ in mesenchymal elements in LR-MDS, we demonstrated increased phosphorylation of $\mathrm{p} 65$, a component of the activated NF- $\mathrm{\kappa B}$ complex, in intramedullary located CD271 ${ }^{+}$mesenchymal cells (Fig. 1d) as well as in bone-lining CD271 ${ }^{+}$stromal cells (Fig. 1e, f). Moreover, pathway analysis (GSEA) confirmed the transcriptional activation of NF- $\mathrm{KB}$ signaling in patients with increased NFKBIA expression in their mesenchymal niche cells (Figure S1A and S1B). NFKBIA expression was significantly correlated with the expression of inflammatory cytokines and negative regulators of hematopoiesis, which are bona fide NF- $\mathrm{KB}$ downstream targets such as IL6, ILS, and CCL3 (Figure $\mathrm{S} 1 \mathrm{C})$. No correlation was found between NFKBIA expression and expression of $S 100 A 8$ or S100A9 (spearman correlation -0.11 and $-0.22 ; P$-adjusted 0.62 and 0.28 , respectively). Patients with activated $\mathrm{NF}-\mathrm{\kappa B}$ signaling $\left(N_{F K B I A}^{+}\right)$in mesenchymal niche cells had no significant difference in overall or progression-free survival in comparison to the $\mathrm{NFKBIA}^{-}$subset (Figure S2A and S2B) in this cohort of uniformly treated LR-MDS patients [4]. No significant correlations were found between mutational status and activation of NF- $\mathrm{KB}$ signaling in mesenchymal cells (Figure S2C).

While the genes encoding inflammatory factors and negative regulators of hematopoiesis [4] are bona fide downstream targets of NF- $\mathrm{\kappa B}$ signaling in other experimental settings, we next wanted to provide experimental support for the view that NF- $\mathrm{kB}$ activation specifically in mesenchymal precursor cells results in upregulation of these targets. To this end, we designed a strategy of activating NF- $\mathrm{KB}$ signaling in mesenchymal progenitor cells by stably overexpressing the constitutively active form of IKK2 (FLAG-IKK2SE), a kinase upstream regulator of NF- $\mathrm{KB}$, via a lentiviral vector (Fig. 2a, Figure S3A-C) [7] in OP9 cells. OP9 cells, like CD271 ${ }^{+}$cells [4], express osteolineage commitment markers as well as HSPC regulatory factors and robustly support the expansion of human HSPCs [8]. NF- $\kappa \mathrm{B}$ activation in OP9 cells resulted in overexpression of NFKBIA (Fig. 2c) and canonical NF- $\mathrm{KB}$ downstream negative regulators of hematopoiesis, including $\mathrm{Il6}, \mathrm{Cxcl} 2$ (murine homolog of IL8), Ccl3, Inhba, Fthl, Ltf, Ccl5, and Cxcl4 (Fig. 2b), recapitulating the findings in LR-MDS patients. Similar to the results in OP9 cells, activation of $\mathrm{NF}-\mathrm{KB}$ in human mesenchymal cells (HS5 cell line and expanded bone-marrow-derived primary mesenchymal cells) (Figure S3D) also resulted in upregulation of NF- $\mathrm{kB}$ downstream targets including negative regulators of hematopoiesis such as IL6, IL8, CCL3, S100A9, INHBA, and CCL5 (Figure S3E). Together, the data link the transcriptional landscape of inflammatory alterations in mesenchymal cells to activation of NF- $\mathrm{KB}$ in LR-MDS.

As earlier reported, in human MDS, the majority of $\mathrm{CD}^{+} 4^{+}$HSPCs is in direct contact with $\mathrm{CD} 271^{+}$ mesenchymal cells [9]. To assess the effect of NF- $\mathrm{KB}$ activation in mesenchymal cells on the biology of normal HSPCs, we performed co-culture experiments with bone marrow $\mathrm{CD}^{+} 4^{+}$HSPCs and OP9 cells transduced with IKK2SE or empty vector (EV) (Fig. 2d). Co-culture for 7 days on IKK2SE-transduced OP9 cells resulted in significantly reduced numbers of immunophenotypically defined HSPCs in comparison to EV-transduced mesenchymal cells (Fig. 2d). In addition, reduced HSPC number was reflected in a reduced number of CFU-Cs (Fig. 2e), indicating attenuation of progenitor function in this setting. NF- $\mathrm{KB}$ activation resulted in impaired proliferation of mesenchymal cells in serum-containing culture conditions (Figure S4A) which may contribute to the 
A

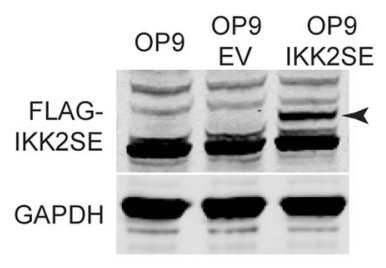

B

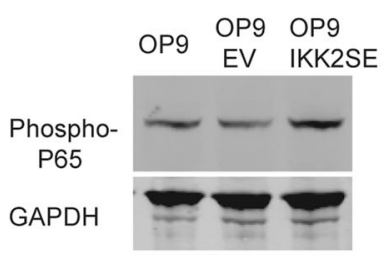

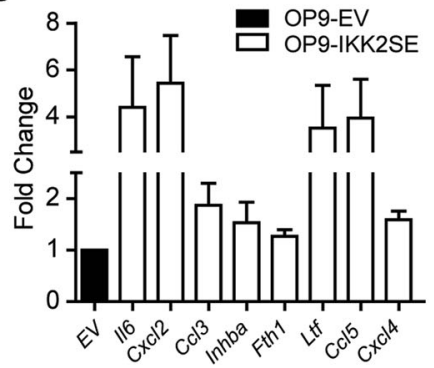

C

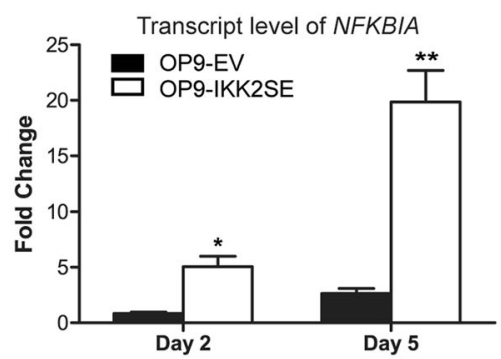

E

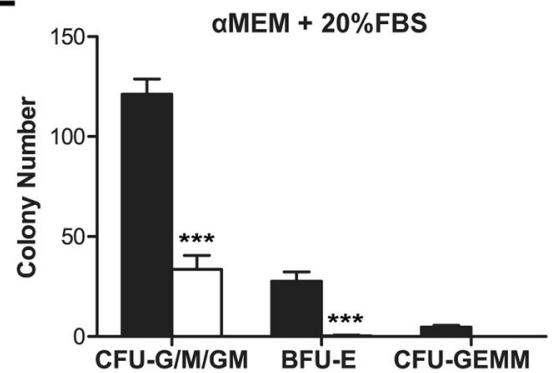

D
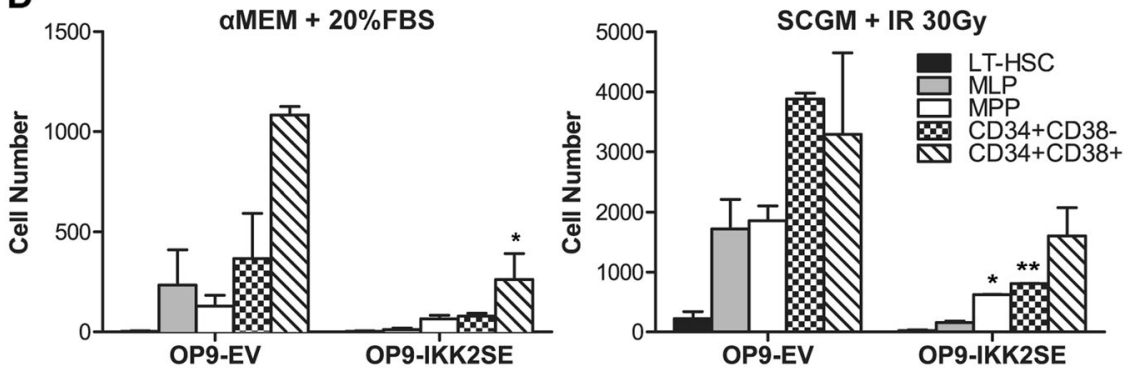

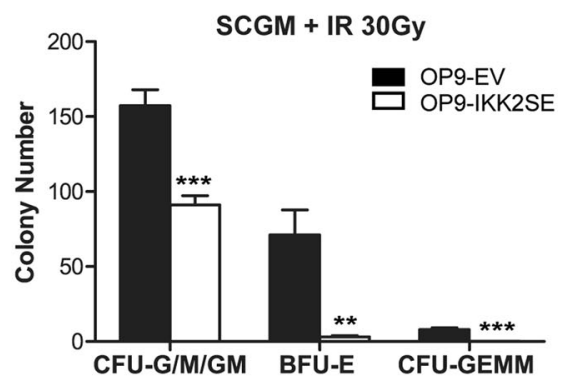

Fig. 2 Activated NF- $\mathrm{B}$ signaling in mesenchymal cells attenuates HSPC number and function. a Western blot analysis showing the overexpression of Flag-IKK2SE and nuclear phospho-p65, the phosphorylated (active) form of $\mathrm{NF}-\kappa \mathrm{B}$, in IKK2SE transduced OP9 cells in comparison to empty vector (EV)-transduced or wild-type cells. b Expression level of NF- $\mathrm{BB}$ downstream targets (Il6, Cxcl2) and disease-relevant negative regulators of hematopoiesis [3, 4] in OP9 cells transduced with IKK2SE. Fold change relative to EV is presented ( $n=3$ for each transcript). c Expression level of NFKBIA in OP9 cells transduced with EV or IKK2SE and re-plated in serum-containing medium for 2 or 5 days. Fold change relative to wildtype OP9 cells is presented $(n=3)$. d Co-culture experiments with bone marrow CD34 ${ }^{+}$ HSPCs and OP9 stromal cells transduced with either EV or IKK2SE. The co-culture took place for 7 days before analysis. The absolute

reduced support for HSPCs in vitro (Fig. 2d, left panel). However, equalizing mesenchymal cell numbers by arresting their proliferation (using irradiation and maintenance in non-proliferative, serum free, culture conditions) (Figure S4B) recapitulated the significantly reduced numbers of immunophenotypically defined HSPCs on IKK2SEtransduced OP9 cells in comparison to EV-transduced mesenchymal cells (Fig. 2d, right panel). This included the fraction enriched for long-term hematopoietic stem cells (LT-HSCs) defined by the markers $\mathrm{CD} 34^{+} \mathrm{CD} 38^{-} \mathrm{CD} 45 \mathrm{RA}$ ${ }^{-} \mathrm{CD} 90^{+}$, multipotent progenitor (MPP; $\mathrm{CD} 34^{+} \mathrm{CD} 38$

number of immunophenotypically defined HSPC subsets ( Lin $^{-} \mathrm{CD} 34$ ${ }^{+} \mathrm{CD} 38^{+}$progenitors, $\mathrm{Lin}^{-} \mathrm{CD} 34^{+} \mathrm{CD} 38^{-} \mathrm{HSPCs}, \mathrm{Lin}^{-} \mathrm{CD} 34^{+} \mathrm{CD} 38$ ${ }^{-} \mathrm{CD} 45 \mathrm{RA}^{-} \mathrm{CD}^{+}{ }^{+}$LT-HSCs, $\mathrm{Lin}^{-} \mathrm{CD} 34^{+} \mathrm{CD} 38^{-} \mathrm{CD}^{-}$RA ${ }^{-} \mathrm{CD} 90^{-}$ MPPs, $\left.\mathrm{Lin}^{-} \mathrm{CD} 34^{+} \mathrm{CD} 38^{-} \mathrm{CD} 45 \mathrm{RA}^{+} \mathrm{CD} 90^{-} \mathrm{MLPs}\right)$ on day 7 after coculturing with OP9-EV or OP9-IKK2SE, in serum-containing medium condition (left panel) and serum-free, stem cell growth medium (SCGM) condition with OP9-EV and OP9-IKK2SE irradiated at $30 \mathrm{~Gy}$ (right panel). Data represent mean \pm SEM of two independent experiments. e The total number of CFU-C after 7 days co-culture in serumcontaining medium condition (left panel) and serum-free medium condition with OP9-EV and OP9-IKK2SE irradiated at $30 \mathrm{~Gy}$ (right panel). Data represent mean \pm SEM of two independent experiments performed in triplicate. Unpaired $t$-test was performed for statistical analysis; $* * * P<.001, * * P<.01, * P<.05$

${ }^{-} \mathrm{CD} 45 \mathrm{RA}^{-} \mathrm{CD}^{-} 0^{-}$), multilymphoid progenitor (MLP; $\mathrm{CD} 34^{+} \mathrm{CD} 38^{-} \mathrm{CD}^{-} 5 \mathrm{RA}^{+} \mathrm{CD} 90^{-}$), as well as primitive $\left(\mathrm{CD} 34^{+} \mathrm{CD} 38^{-}\right)$and committed $\mathrm{CD} 34^{+} \mathrm{CD} 38^{+}$progenitor cells (Fig. 2d), indicating that mesenchymal NF-kB signaling attenuates HSPC number and function, at least partially, independent of its effect on mesenchymal cell proliferation.

Collectively, in this brief communication, we demonstrate that mesenchymal NF- $\mathrm{KB}$ activation is a common finding in LR-MDS patients leading to transcriptional upregulation of inflammatory programs associated with 
negative regulation of hematopoiesis and attenuation of HSPC numbers and function.

Demonstration of mesenchymal activation of NF- $\mathrm{KB}$ provides human disease relevance to a number of murine studies implicating NF- $\mathrm{KB}$ activation in ancillary cells to the pathogenesis of hematopoietic disease. NF- $\mathrm{KB}$ activation in non-hematopoietic cells has been shown to induce 'MDSlike' myeloproliferative disease (MPD) in mice [10]. In another study, NF- $\mathrm{BB}$ activation in bone marrow mesenchymal cells and endothelial cells, as a result of elevated levels of the microRNA miR-155, generated a persistent pro-inflammatory state of the bone marrow niche leading to an MPD-like disease in a Notch/RBPJ loss-offunction mouse model [11]. Our experimental data demonstrate that NF- $\mathrm{\kappa B}$ activation in the mesenchyme attenuates normal hematopoiesis, which is of key relevance to LR-MDS characterized by cytopenia. The implication of mesenchymal NF- $\mathrm{kB}$ activation in the pathogenesis of MDS may also point toward common mechanisms between the pathogenesis of MDS and oncogenesis in other systems. NF- $\kappa B$-mediated chronic tissue inflammation has been shown to drive cancer initiation and progression via secretion of cytokines and soluble factors in models of several other forms of cancer [12,13], including skin, prostate, and colon cancer. In these models, activation of NF- $\mathrm{KB}$ signaling, specifically in fibroblasts, promoted malignant features in heterotypic (pre)cancerous cells, supporting the hypothesis that mesenchymal inflammation may facilitate tumorigenesis in the hematopoietic system as well.

The activation of NF- $\mathrm{KB}$ signaling in mesenchymal cells in most LR-MDS patients raises intriguing questions about the events driving this activation. This includes the question whether mesenchymal cell-intrinsic alterations or extrinsic events are driving NF- $\mathrm{kB}$ activation. While the answer to this question remains speculative in the absence of experimental evidence (and may vary between patients), it is conceivable that primary alterations in hematopoietic elements drive activation of NF- $\mathrm{kB}$ activation in the mesenchymal niche.

This notion is supported by recent findings where activation of the NF- $\mathrm{KB}$ pathway in CD34 ${ }^{+}$HSPCs is implicated in MDS pathogenesis [14] and CD271 ${ }^{+}$mesenchymal cells colocalize with $\mathrm{CD} 34^{+}$HSPCs in the bone marrow section of MDS patients [9]. It is therefore reasonable to hypothesize that activated NF- $\mathrm{KB}$ pathway in HSPCs signals to the adjacent mesenchymal elements, resulting in NF- $\mathrm{KB}$ activation in mesenchymal cells. As NF- $\mathrm{KB}$ activation is likely maintained through autocrine/paracrine feedback signaling networks, other cellular types that anatomically localize with the activated HSPCs and mesenchymal elements could be involved as well, suggesting that diverse cellular components may participate in this crosstalk. The combined findings suggest that in LR-MDS, activation of NF-kB occurs in both hematopoietic and mesenchymal cells, likely through autocrine and paracrine feedback signaling networks, leading to a NF-кBmediated inflammatory milieu in the LR-MDS bone marrow and an overexpression of a repertoire of secreted negative hematopoietic regulators. S100A8/9, recently shown by us to induce NF- $\mathrm{KB}$ activation and genotoxic stress in HSPCs and to be associated with an increased likelihood of leukemic transformation, was not correlated with NFKBIA expression in patients, indicating that its regulation is more complex and may include upstream TP53 activation [3].

Taken together, the findings support the notion that mesenchymal factors, in addition to hematopoietic cell autonomous characteristics, may be therapeutically targeted in LR-MDS and warrant ongoing experiments defining the contribution of NF- $\mathrm{KB}$ activation and inflammation to ineffective hematopoiesis and leukemic evolution in MDS.

Acknowledgements The authors thank O. Roovers, P.W.M. Vermeulen, P. van Geel, and Dr. W.J.C. Chikhovskaya-Rombouts for their technical support, Dr. K. Lam for providing healthy and MDS bone marrow biopsies, Dr. ir. D.A. Chitu of the HOVON data center for providing clinical data and general statistical advice, Prof. J.H. Jansen for performing mutational studies and providing molecular data of the patients, Dr. H. Schepers and Prof. J.J. Schuringa for providing the retroviral construct pMIG HA-IKK-2 S177E S181E, and Prof. I. Touw for helpful comments on the manuscript. Part of this work is presented in the doctoral thesis 'The mesenchymal niche in leukemia predisposition syndromes' by SC.

Funding This work was supported by grants from the Dutch Cancer Society (KWF Kankerbestrijding) (EMCR 2016-10488), the Netherlands Organization of Scientific Research (NWO 90700422), and the Netherlands Genomics Initiative (40-41009-98-11062) to MHGPR.

Author contributions ZP, SC, and MHGPR designed the studies; ZP, SC, SJFH, KK, JF, CVD, EMJB, AMM, and DJL-K performed the experiments and acquired the data; SC, RMH, EMJB, and MAS provided technical guidance and bioinformatical analysis; JNS provided helpful insights in immunohistochemical data analysis; EMPC and AvdL provided the patient material; ZP, SC, and MHGPR wrote the manuscript. All authors were involved in data interpretation and manuscript reviewing; MHGPR supervised the study.

\section{Compliance with ethical standards}

Conflict of interest The authors declare that they have no conflict of interest.

Open Access This article is licensed under a Creative Commons Attribution 4.0 International License, which permits use, sharing, adaptation, distribution and reproduction in any medium or format, as long as you give appropriate credit to the original author(s) and the source, provide a link to the Creative Commons license, and indicate if changes were made. The images or other third party material in this article are included in the article's Creative Commons license, unless indicated otherwise in a credit line to the material. If material is not included in the article's Creative Commons license and your intended use is not permitted by statutory regulation or exceeds the permitted use, you will need to obtain permission directly from the copyright holder. To view a copy of this license, visit http://creativecommons. org/licenses/by/4.0/. 


\section{References}

1. Raaijmakers MH, Mukherjee S, Guo S, Zhang S, Kobayashi T, Schoonmaker JA, et al. Bone progenitor dysfunction induces myelodysplasia and secondary leukaemia. Nature. 2010;464: 852-7.

2. Medyouf H, Mossner M, Jann JC, Nolte F, Raffel S, Herrmann C, et al. Myelodysplastic cells in patients reprogram mesenchymal stromal cells to establish a transplantable stem cell niche disease unit. Cell Stem Cell. 2014;14:824-37.

3. Zambetti NA, Ping Z, Chen S, Kenswil KJ, Mylona MA, Sanders MA, et al. Mesenchymal inflammation drives genotoxic stress in hematopoietic stem cells and predicts disease evolution in human pre-leukemia. Cell Stem Cell. 2016;19:613-27.

4. Chen S, Zambetti NA, Bindels EM, Kenswill K, Mylona AM, Adisty NM, et al. Massive parallel RNA sequencing of highly purified mesenchymal elements in low-risk MDS reveals tissuecontext-dependent activation of inflammatory programs. Leukemia. 2016;30:1938-42.

5. Brown K, Park S, Kanno T, Franzoso G, Siebenlist U. Mutual regulation of the transcriptional activator NF-kappa B and its inhibitor, I kappa B-alpha. Proc Natl Acad Sci USA. 1993;90:2532-6.

6. Sun SC, Ganchi PA, Ballard DW, Greene WC. Nf-Kappa-B controls expression of inhibitor I-kappa-B-alpha—evidence for an inducible autoregulatory pathway. Science. 1993;259:1912-5.

7. Mercurio F, Zhu H, Murray BW, Shevchenko A, Bennett BL, Li J, et al. IKK-1 and IKK-2: cytokine-activated IkappaB kinases essential for NF-kappaB activation. Science. 1997;278:860-6.
8. Vodyanik MA, Bork JA, Thomson JA, Slukvin II. Human embryonic stem cell-derived CD34+ cells: efficient production in the coculture with OP9 stromal cells and analysis of lymphohematopoietic potential. Blood. 2005;105:617-26.

9. Flores-Figueroa E, Varma S, Montgomery K, Greenberg PL, Gratzinger D. Distinctive contact between CD34+ hematopoietic progenitors and CXCL12+CD271+ mesenchymal stromal cells in benign and myelodysplastic bone marrow. Lab Invest. 2012;92:1330-41.

10. Rupec RA, Jundt F, Rebholz B, Eckelt B, Weindl G, Herzinger T, et al. Stroma-mediated dysregulation of myelopolesis in mice lackingk 1 kappa B alpha. Immunity. 2005;22:479-91.

11. Wang L, Zhang H, Rodriguez S, Cao L, Parish J, Mumaw C, et al. Notch-dependent repression of miR-155 in the bone marrow niche regulates hematopoiesis in an NF-kappaB-dependent manner. Cell Stem Cell. 2014;15:51-65.

12. DiDonato JA, Mercurio F, Karin M. NF-kappaB and the link between inflammation and cancer. Immunol Rev. 2012;246:379400.

13. Pikarsky E, Porat RM, Stein I, Abramovitch R, Amit S, Kasem S, et al. NF-kappaB functions as a tumour promoter in inflammationassociated cancer. Nature. 2004;431:461-6.

14. Wei Y, Chen R, Dimicoli S, Bueso-Ramos C, Neuberg D, Pierce $\mathrm{S}$, et al. Global H3K4me3 genome mapping reveals alterations of innate immunity signaling and overexpression of JMJD3 in human myelodysplastic syndrome CD34+ cells. Leukemia. 2013;27:2177-86.

\title{
Targeting MEK in vemurafenib-resistant hairy cell leukemia
}

\author{
Rebecca Caeser ${ }^{1,2} \cdot$ Grace Collord $^{3,4} \cdot$ Wen-Qing $\mathrm{Yao}^{5} \cdot$ Zi Chen $^{5} \cdot$ George S. Vassiliou $\mathbb{1}^{1,3} \cdot$ Philip A. Beer $^{3} \cdot$ \\ Ming-Qing Du ${ }^{5} \cdot$ Mike A. Scott $^{6} \cdot$ George A. Follows ${ }^{7}$ - Daniel J. Hodson ${ }^{1,2,7}$
}

Received: 17 July 2018 / Revised: 13 August 2018 / Accepted: 20 August 2018 / Published online: 19 October 2018

(C) The Author(s) 2018. This article is published with open access

These authors contributed equally: Rebecca Caeser, Grace Collord.

Electronic supplementary material The online version of this article (https://doi.org/10.1038/s41375-018-0270-2) contains supplementary material, which is available to authorized users.

Daniel J. Hodson

djh1002@cam.ac.uk

1 Department of Haematology, University of Cambridge,

Cambridge, UK

2 Wellcome-MRC Cambridge Stem Cell Institute, Cambridge, UK

3 Wellcome Sanger Institute, Hinxton, UK

4 Department of Paediatrics, University of Cambridge,
Hairy cell leukemia (HCL) is a chronic, incurable B cell malignancy that presents with splenomegaly, bone marrow infiltration, and cytopenias [1]. Long remissions are typically achieved with purine analogs such as cladribine, but most cases will relapse and require further therapy. The discovery of the

Cambridge, UK

5 Division of Molecular Histopathology, University of Cambridge, Cambridge, UK

6 Haematopathology \& Oncology Diagnostic Service, Cambridge University Hospitals, Cambridge, UK

7 Department of Haematology, Cambridge University Hospitals, Cambridge, UK 


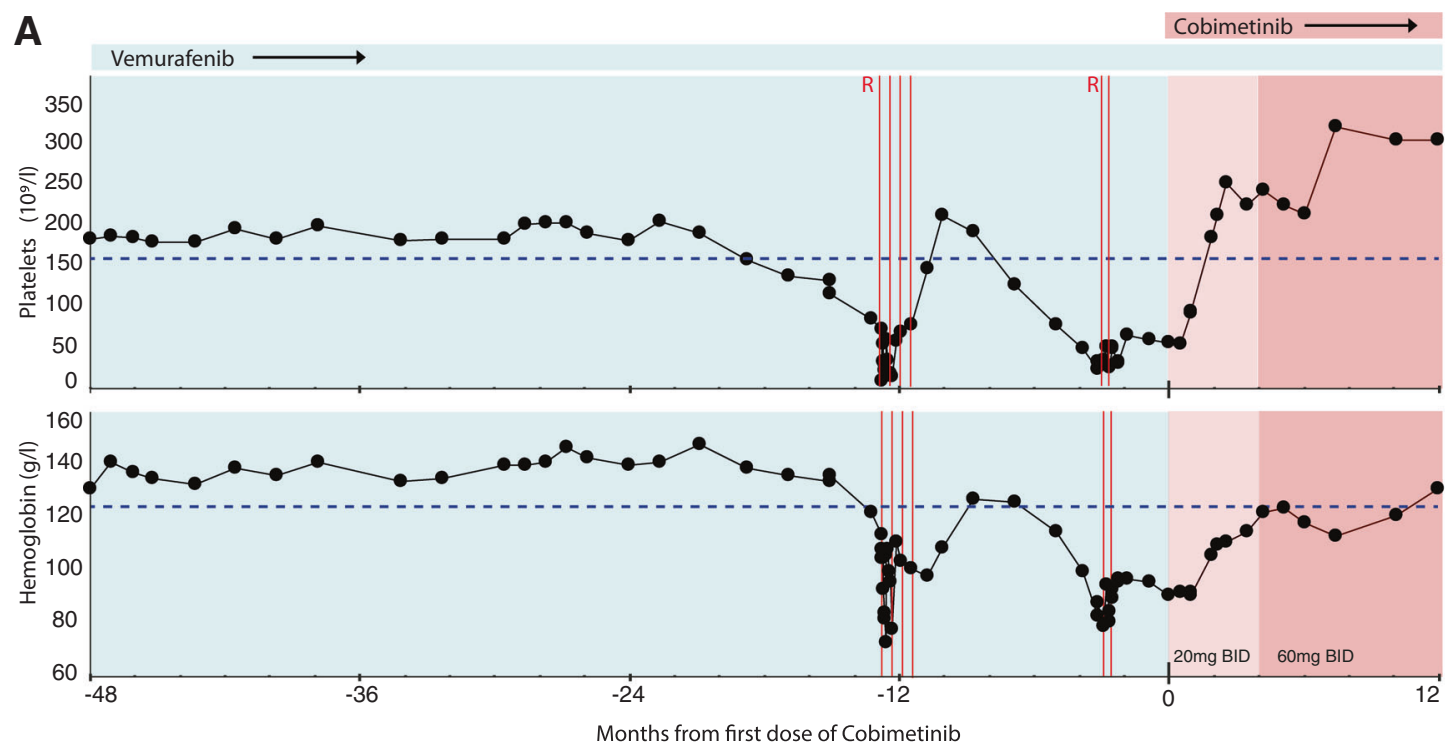

B

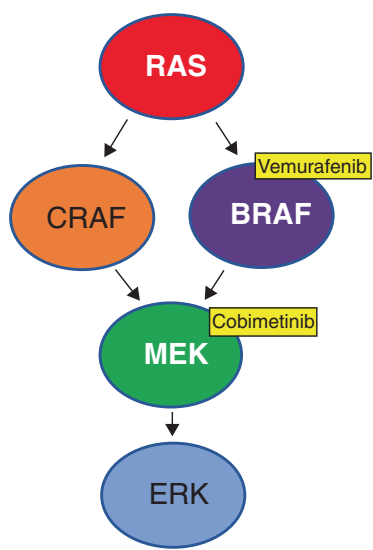

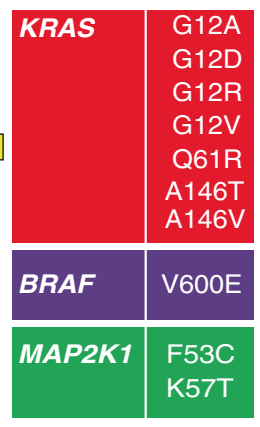

G12R

G12V

$146 \mathrm{~V}$

(5)

$57 \mathrm{~T}$

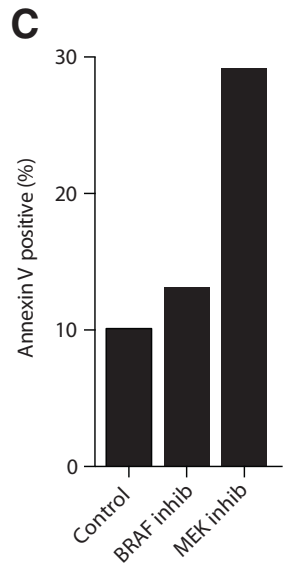

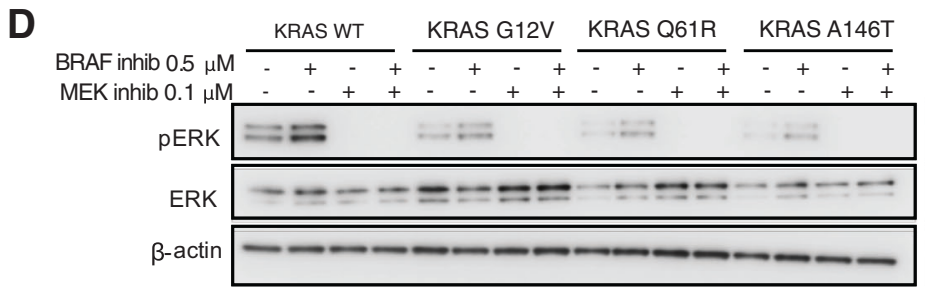

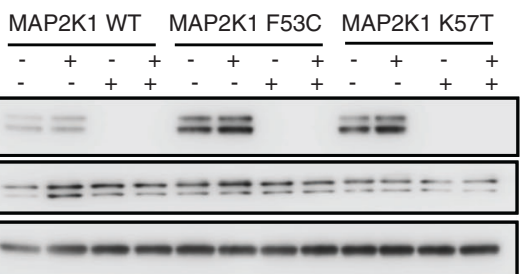

Fig. 1 a The patient's peripheral blood indices are shown over time relative to the first dose of the MEK inhibitor cobimetinib. Vertical red lines indicate the timing of rituximab dosing. Blue shading indicates vemurafenib monotherapy $240 \mathrm{mg}$ twice daily (vem mono). Pale pink shading indicates vemurafenib with cobimetinib $20 \mathrm{mg}$ daily (cobi-20). Darker pink indicates vemurafenib with cobimetinib $60 \mathrm{mg}$ daily (21/ 28 days) (cobi-60). The lower limits of normal reference values are indicated by horizontal dashed lines. b Schematic of the MEK-ERK signaling pathway with mutations identified in purified tumor cells

$B R A F$ V600E mutation in almost all cases of HCL [2] has led to the widespread adoption of the BRAF inhibitor vemurafenib for treatment of patients relapsing after cladribine [3-5]. Impressive responses are reported; however, relapse is inevitable $[5,6]$ and hematologists are now faced with a growing after emergence of resistance to vemurafenib. c Annexin V staining was used to quantify the induction of apoptosis in tumor cells purified from the patient and incubated for $48 \mathrm{~h}$ ex vivo with inhibitors of BRAF (vemurafenib) or MEK (trametinib). Apoptosis is induced by MEK inhibition but not by BRAF inhibition. d Immunoblots of a lymphoma cell line transduced with the indicated KRAS or MAP2K1 constructs and incubated with inhibitors of BRAF or MEK. Complete suppression of ERK activity is seen with MEK inhibition but not with BRAF inhibition

number of patients with vemurafenib-resistant HCL. The optimal management of these patients remains unclear.

The molecular basis of vemurafenib resistance has been extensively investigated in recent years in patients with $B R A F$ mutant solid organ malignancies such as melanoma 


\section{A}
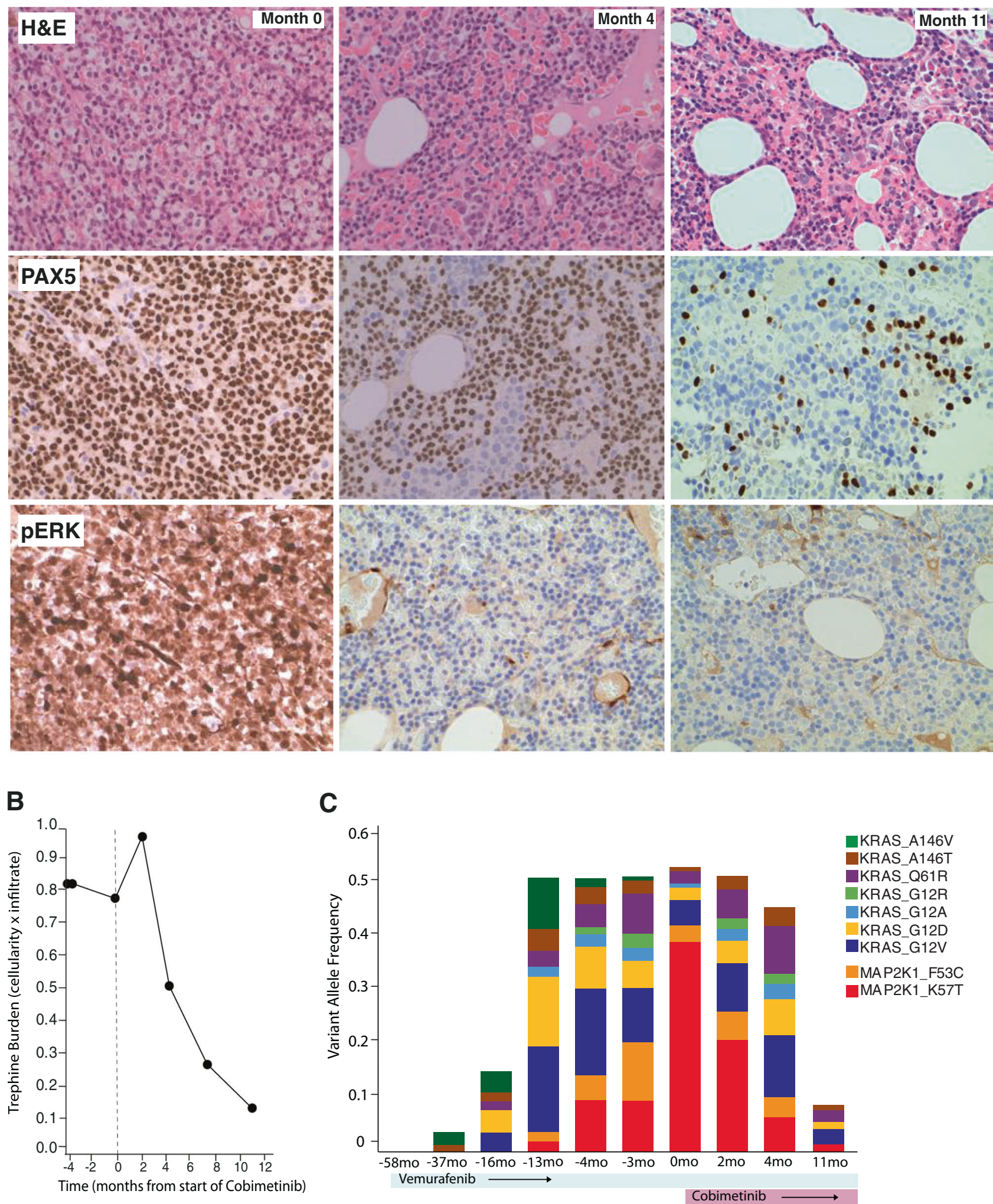

Fig. 2 a Bone marrow trephine biopsies stained with H\&E (top) or PAX5 antibody (middle) or pERK (lower) taken at the indicated time points relative to start of cobimetinib. $\mathbf{b}$ Leukemic burden prior to and after starting cobimetinib therapy was calculated as the product of

bone marrow trephine cellularity and leukemic cell infiltrate. c Mutant allele frequency for the indicated $K R A S$ and $M A P 2 K 1$ mutations quantified by targeted amplicon sequencing at multiple time point relative to treatment 
and colorectal cancer [7]. Resistance to vemurafenib in melanoma frequently results from reactivation of ERK pathway signaling by a variety of genetic mechanisms that include activating mutations of NRAS or KRAS, amplification of mutant $B R A F$, aberrant splicing of $B R A F$, and activating mutation of $M A P 2 K 1$, which encodes the MEK1 protein [7, 8]. ERK-independent mechanisms are less frequent and include activation of PI3K signaling [7]. The predominance of genetic mechanisms converging on ERK reactivation has led to the successful use of dual MEK/ BRAF inhibition in melanoma [9]. In colorectal cancer however, different mechanisms apply; here primary resistance usually results from reduced feedback inhibition upon upstream receptor tyrosine kinase activity leading to restoration of ERK activity [10]. In this scenario, combined BRAF and MEK inhibition has not proved effective [11].

In contrast to our comprehensive understanding in solid organ cancer, very little is known about the mechanistic basis of vemurafenib resistance in HCL. Given the diversity of resistance mechanisms established in other cancers, it is unclear which, if any, of these might predominate in HCL. Two acquired subclonal, activating KRAS mutations were previously reported in a single patient with vemurafenib resistance [5]. Deletions of $N F 1$ and NF2 have been proposed as an alternative mechanism in another case of primary resistance [12]. The use of MEK inhibition has been suggested as a logical therapeutic strategy in patients who have reactivated ERK signaling. However, the use of MEK inhibition has never previously been reported in a patient with HCL and at present there is no consensus on the optimal management of patients relapsing on vemurafenib.

A 74-year-old patient with HCL had been treated at our institution with splenectomy, cladribine, and pentostatin. We previously reported his initial response to vemurafenib at a dose of $240 \mathrm{mg}$ twice daily [4]. This dose was lower than used in the initial phase II trial [5], but has since been shown in several reports to be an effective dosing strategy for HCL $[3,13,14]$. Vemurafenib was initially stopped after 58 days; however, this was associated with rapid return of marrow infiltration and thrombocytopenia. Vemurafenib was restarted at the same dose and cytopenias rapidly resolved. Continuous low-dose vemurafenib continued to sustain his remission for over 3 years, attesting to the efficacy of this dosing schedule. However, 38 months after restarting vemurafenib, his blood indices deteriorated, and he required platelet transfusion (Fig. 1a). Bone marrow trephine biopsy confirmed relapse of HCL. A trial of rituximab with continued vemurafenib led to transient recovery of hematological indices. However, bone marrow infiltration did not improve over the next 4 months, and the patient became anemic, thrombocytopenic, and required further platelet transfusion. A second trial of two doses of rituximab produced a minimal improvement of platelet count to $30 \times 10^{9} / 1$. The patient became systemically unwell with B symptoms. Bone marrow trephine biopsy confirmed 99\% infiltration with HCL.

To elucidate the mechanism of his resistance we performed whole-genome and deep-targeted sequencing of 292 genes (Supplementary Table 1) of DNA from purified tumor cells collected prior to starting vemurafenib and again at relapse. Samples were used with informed written patient consent in accordance with the Declaration of Helsinki and appropriate institutional ethical approvals. Sequencing studies revealed the presence of the known BRAF V600E mutation and chromosome 7q deletion. Remarkably, we also identified seven distinct activating mutations in KRAS and two mutations in MAP2K1 (encoding MEK1) (Fig. 1b and Supplementary Table 2). These were detectable at relapse but were not detectable prior to vemurafenib exposure. Allele frequencies were consistent with the parallel, convergent evolution of multiple clones. Deep-targeted amplicon sequencing at multiple time points showed how KRAS mutations developed early, initially with codon 146 mutations, followed by emergence of the more classical activating mutations of codons 12 and 61 [15]. MAP2K1 mutations appeared later with $M A P 2 K 1$ p.K57T expanding to become the dominant clone (Fig. 2c and Supplementary Table 2). The convergent nature of these mutations strongly implicated reactivation of MEK-ERK signaling as the likely mechanism of resistance. Indeed, immunohistochemistry confirmed strong pERK activity in all tumor cells (Fig. 2a). We looked for other mechanisms of resistance reported in melanoma. Specifically, we found no genetic or protein evidence of either increased pAKT activity or altered BRAF splicing (Supplementary Figure 1A \& B).

We concluded that reactivation of MEK/ERK activity was the most likely driver of relapse and hypothesized that MEK inhibition might be a successful therapeutic strategy. Expression of the KRAS and MAP2K1 mutants in a lymphoid cell line showed that while each mutation was able to activate ERK in the presence of vemurafenib, all mutations remained sensitive to MEK inhibition (Fig. 1d). Exposure of the patient's purified tumor cells to vemurafenib ex vivo failed to completely suppress ERK activity and did not induce apoptosis. In contrast, ERK activity was completely suppressed and apoptosis induced by exposure to a MEK inhibitor (Supplementary Figure 1C and Fig. 1c).

Based on our in vitro experiments, we treated the patient with the MEK inhibitor cobimetinib, initially at $20 \mathrm{mg}$ daily combined with vemurafenib $240 \mathrm{mg}$ twice daily. Remarkably, B symptoms resolved within 1 week, followed by rapid platelet count recovery. A bone marrow biopsy at 4 months showed complete suppression of ERK activity (Fig. 2a). However, HCL marrow infiltration persisted, and therefore cobimetinib dose was increased to $60 \mathrm{mg}$ daily (taken 21 out of 28 days). The dose was well tolerated and 
was associated with further resolution of cytopenias, a substantial reduction in bone marrow cellularity and HCL infiltration, ongoing suppression of ERK activity and restoration of normal hematopoiesis (Fig. 2a, b). Ongoing treatment was also associated with suppression of mutant allele frequencies for $B R A F, K R A S$, and $M A P 2 K 1$ mutations (Fig. 2c). At 12 months, the patient remains well and asymptomatic with continued combination therapy.

The finding of nine activating mutations, all converging upon the activation of RAS/RAF/MEK/ERK signaling, underscores the centrality of this pathway in HCL and its reactivation as a potent mechanism of resistance to vemurafenib in this disease. This report provides a detailed analysis of the molecular basis for acquired vemurafenib resistance in HCL. It is the first reported use of a MEK inhibitor to treat vemurafenib-resistant HCL. It proposes a new therapeutic option for such patients and provides impetus for testing in a formal trial setting.

Acknowledgements DJH was personally supported by a Clinician Scientist Fellowship from the Medical Research Council (MR/ M008584/1), GC by a Wellcome Clinical PhD Fellowship (WT098051). W-QY was supported by an International Collaboration Award from the Pathological Society of UK and Ireland. Research in M.D. lab was supported by grants from Bloodwise. Core support was received from the Cancer Research UK, Cambridge Cancer Centre. We thank Joanna Baxter and Cambridge Blood and Stem Cell Bank for sample collection and storage, and Calli Latimer and Claire Hardy for expert technical assistance.

Author contributions RC designed and performed the experiments. GC analyzed the whole-genome sequencing and cRNA bait pulldown data. PAB designed the targeted gene pulldown panel. W-QY and ZC designed and conducted the targeted amplicon sequencing and analyzed the results. MSA, GAF, M-QD, GSV, and PAB provided clinical and diagnostic expertise and contributed to data interpretation. DJH designed the experiments, provided clinical expertise, directed the research, and wrote the manuscript with contributions from RC and GC.

\section{Compliance with ethical standards}

Conflict of interest DJH research funding: Gilead Sciences. GAF honoraria: Bayer AG, Roche, Gilead Sciences, Janssen Pharmaceuticals, and AbbVie. Consulting or advisory role: Bayer AG, Roche, Gilead Sciences, Janssen Pharmaceuticals, AbbVie. Speakers' bureau: Bayer AG, Roche, Gilead Sciences, Janssen Pharmaceuticals, and AbbVie. PAB employment: Karus.

Open Access This article is licensed under a Creative Commons Attribution 4.0 International License, which permits use, sharing, adaptation, distribution and reproduction in any medium or format, as long as you give appropriate credit to the original author(s) and the source, provide a link to the Creative Commons license, and indicate if changes were made. The images or other third party material in this article are included in the article's Creative Commons license, unless indicated otherwise in a credit line to the material. If material is not included in the article's Creative Commons license and your intended use is not permitted by statutory regulation or exceeds the permitted use, you will need to obtain permission directly from the copyright holder. To view a copy of this license, visit http://creativecommons. org/licenses/by/4.0/.

\section{References}

1. Falini B, Martelli MP, Tiacci E. BRAF V600E mutation in hairy cell leukemia: from bench to bedside. Blood. 2016;128:1918-27.

2. Tiacci E, Trifonov V, Schiavoni G, Holmes A, Kern W, Martelli $\mathrm{MP}$, et al. BRAF mutations in hairy-cell leukemia. N Engl J Med. 2011;364:2305-15.

3. Dietrich S, Glimm H, Andrulis M, von Kalle C, Ho AD, Zenz T. BRAF inhibition in refractory hairy-cell leukemia. N Engl J Med. 2012;366:2038-40.

4. Follows GA, Sims H, Bloxham DM, Zenz T, Hopper MA, Liu H, et al. Rapid response of biallelic BRAF V600E mutated hairy cell leukaemia to low dose vemurafenib. $\mathrm{Br} \mathrm{J}$ Haematol. 2013;161:150-3.

5. Tiacci E, Park JH, De Carolis L, Chung SS, Broccoli A, Scott S, et al. Targeting mutant BRAF in relapsed or refractory hairy-cell leukemia. N Engl J Med. 2015;373:1733-47.

6. Holderfield M, Deuker MM, McCormick F, McMahon M. Targeting RAF kinases for cancer therapy: BRAF-mutated melanoma and beyond. Nat Rev Cancer. 2014;14:455-67.

7. Shi H, Hugo W, Kong X, Hong A, Koya RC, Moriceau G, et al. Acquired resistance and clonal evolution in melanoma during BRAF inhibitor therapy. Cancer Discov. 2014;4:80-93.

8. Poulikakos PI, Persaud Y, Janakiraman M, Kong X, Ng C, Moriceau $\mathrm{G}$, et al. RAF inhibitor resistance is mediated by dimerization of aberrantly spliced BRAF(V600E). Nature. 2011;480: 387-90.

9. Larkin J, Ascierto PA, Dreno B, Atkinson V, Liszkay G, Maio M, et al. Combined vemurafenib and cobimetinib in BRAF-mutated melanoma. N Engl J Med. 2014;371:1867-76.

10. Corcoran RB, Ebi H, Turke AB, Coffee EM, Nishino M, Cogdill $\mathrm{AP}$, et al. EGFR-mediated re-activation of MAPK signaling contributes to insensitivity of BRAF mutant colorectal cancers to RAF inhibition with vemurafenib. Cancer Discov. 2012;2: 227-35.

11. Kopetz S, Desai J, Chan E, Hecht JR, O'Dwyer PJ, Maru D, et al. Phase II pilot study of vemurafenib in patients with metastatic BRAF-mutated colorectal cancer. J Clin Oncol. 2015;33:4032-8.

12. Durham BH, Getta B, Dietrich S, Taylor J, Won H, Bogenberger $\mathrm{JM}$, et al. Genomic analysis of hairy cell leukemia identifies novel recurrent genetic alterations. Blood. 2017;130:1644-8.

13. Peyrade F, Re D, Ginet C, Gastaud L, Allegra M, Ballotti R, et al. Low-dose vemurafenib induces complete remission in a case of hairy-cell leukemia with a V600E mutation. Haematologica. 2013;98:e20-2.

14. Dietrich S, Pircher A, Endris V, Peyrade F, Wendtner CM, Follows GA, et al. BRAF inhibition in hairy cell leukemia with lowdose vemurafenib. Blood. 2016;127:2847-55.

15. Janakiraman M, Vakiani E, Zeng Z, Pratilas CA, Taylor BS, Chitale D, et al. Genomic and biological characterization of exon 4 KRAS mutations in human cancer. Cancer Res. 2010;70: 5901-11. 
Leukemia (2019) 33:546-549

https://doi.org/10.1038/s41375-018-0272-0

Multiple myeloma gammopathies

\title{
The majority of newly diagnosed myeloma patients do not fulfill the inclusion criteria in clinical phase III trials
}

\author{
Tobias W. Klausen ${ }^{1} \cdot$ Henrik Gregersen ${ }^{2} \cdot$ Niels Abildgaard ${ }^{3} \cdot$ Niels Frost Andersen $^{4} \cdot$ Ulf Christian Frølund $^{5} \cdot$ \\ Peter Gimsing ${ }^{6} \cdot$ Carsten Helleberg $^{1} \cdot$ Annette J. Vangsted ${ }^{6}{ }^{6}$
}

Received: 31 May 2018 / Revised: 18 August 2018 / Accepted: 3 September 2018 / Published online: 28 September 2018

๑) Springer Nature Limited 2018

Real-world data (RWD) are important as randomized clinical trials (RCT) have strict in- and exclusion criteria that do not allow evaluation of the effect and toxicity of new drugs or treatment modalities in patients that do not fulfill these criteria. Since 2005, clinical data on all newly diagnosed multiple myeloma (MM) patients have been registered in the Danish Multiple Myeloma Registry (DMMR) [1, 2]. The Danish National guidelines for treatment of MM, are based on the results of phase III RCT (www.myeloma.dk).

The median overall survival (OS) for patients above 65 years registered in DMMR in 2009-2014 is less as compared to the Hovon 87 trial (32.9 months (mo.) versus 48 mo.) [3, 4]. In patients eligible for high-dose melphalan with autologous stem cell transplantation (HDM-ASCT) the median OS in the DMMR was 74 mo. in 2009-2014 (Abstract 1946 EHA 2016). A review of OS for patients treated in HDM-ASCT RCT describe a 3-year OS of $80 \%$ and $75 \%$ in patients treated with and without bortezomib, respectively [5]. In the DMMR, 3-year OS was $77 \%$ in the

Electronic supplementary material The online version of this article (https://doi.org/10.1038/s41375-018-0272-0) contains supplementary material, which is available to authorized users.

Annette J. Vangsted

annette.juul.vangsted@ regionh.dk

1 Department of Hematology, Herlev Hospital, Herlev, Denmark

2 Department of Hematology, Aalborg University Hospital, Aalborg, Denmark

3 Department of Hematology, Odense University Hospital, Odense, Denmark

4 Department of Hematology, Aarhus University Hospital, Aarhus, Denmark

5 Department of Hematology, Roskilde Hospital, Roskilde, Denmark

6 Department of Hematology, Rigshospitalet, Copenhagen, Denmark calendar period 2009-2013 [4]. The worse OS of the elderly in the DMMR let us to compare OS for patients registered in DMMR who fulfilled the inclusion and exclusion criteria in phase III RCT with OS for those patients who did not. Furthermore, we analyzed the fraction of population-based cohort of newly diagnosed elderly and younger myeloma patients that fulfill the inclusion and exclusion criteria.

Four recent phase III RCTs for MM patients ineligible for HDM-ASCT (Hovon 87 trial [3]; VISTA trial [6]; FIRST trial [7]; and the SWOG S0777 trial [8]) and four recent phase III RCTs in MM patients eligible for HDMASCT at diagnosis (STaMINA trial [9]; IFM2013-04 trial [10]; IFM DFCI 2009 trial [11], and the Hovon95/EMN02 trial [12]) were used and 11 inclusion and exclusion criteria, reported in at least 3 out of 4 trials, were selected (Supplementary Tables S1 and S2). The three inclusion criteria were $\mathrm{CRAB}$, measurable disease, and bone marrow plasma cells (PLC) $\geq 10 \%$, and the eight exclusion criteria were kidney failure, WHO performance status (PS) $>2$, AL amyloidosis, acute myocardial infarction (AMI) within last 6 months, human immunodeficiency virus (HIV), hepatitis $\mathrm{B}$ or $\mathrm{C}$, cancer within 5 years (excluding stage $0-1$ cervical cancer, some skin cancers), severe comorbidity (congestive heart failure $(\mathrm{CHF})$, dementia, severe diabetes, moderate, or severe liver disease, hemiplegia) [2] (Supplementary Table S3).

The validated DMMR has been described [1]. ICD-10 codes from the Danish National Patient Registry (DNPR) were used (Supplementary Table S3) [2].

In the relapse setting, thalidomide was introduced in 1999 , bortezomib in 2003, lenalidomide in 2005 , pomalidomide in 2014, carfilzomib, elotuzumab, and daratumumab in 2016, and ixazomib in 2017 [13, 14]. Combination treatment including thalidomide, bortezomib, and lenalidomide are now treatment options at diagnosis.

Clinical data from $2189 \mathrm{MM}$ patients at diagnosis were collected from DMMR and DNPR between 1 January 2005 and 31 December 2013 (Supplementary Table S3) [1, 2]. 
Table 1 Distribution and effect on overall survival of inclusion and exclusion criteria for patients registered in DMMR

\begin{tabular}{|c|c|c|c|c|}
\hline \multirow[t]{2}{*}{ Criteria } & \multicolumn{2}{|c|}{ Distribution of criteria } & \multicolumn{2}{|c|}{ Effect on OS dependent on criteria } \\
\hline & Fulfill IC & No fulfillment of IC & HR $(95 \%$ CI $)$ & $P$ \\
\hline \multicolumn{5}{|l|}{$>65(N=1425)$} \\
\hline \multicolumn{5}{|c|}{ Hovon 87, VISTA, FIRST, SWOG S0777 trials } \\
\hline CRAB & $1339(94.0 \%)$ & $86(6.0 \%)$ & $0.9(0.7-1.2)$ & 0.55 \\
\hline Measurable disease & $1188(95.7 \%)$ & $54(4.3 \%)$ & $0.7(0.5-1.0)$ & 0.031 \\
\hline PLC $<10 \%$ & $1208(88.6 \%)$ & $156(11.4 \%)$ & $0.7(0.6-0.9)$ & 0.0009 \\
\hline Kidney failure & $1036(73.9 \%)$ & $365(26.1 \%)$ & $1.7(1.5-1.9)$ & $<0.0001$ \\
\hline $\mathrm{PS}>2$ & $1192(83.6 \%)$ & $233(16.4 \%)$ & $2.9(2.5-3.4)$ & $<0.0001$ \\
\hline Amyloidosis & $1397(98.0 \%)$ & $28(2.0 \%)$ & $1.6(1.0-2.3)$ & 0.029 \\
\hline AMI within 6 mo. & $1391(97.6 \%)$ & $34(2.4 \%)$ & $2.2(1.5-3.1)$ & $<0.0001$ \\
\hline HIV & $1425(100.0 \%)$ & $0(0.0 \%)$ & - & - \\
\hline Hepatitis & $1421(99.7 \%)$ & $4(0.3 \%)$ & $2.5(0.9-6.6)$ & 0.072 \\
\hline Cancer within $5 \mathrm{Y}$ & $1277(89.6 \%)$ & $148(10.4 \%)$ & $1.1(0.9-1.4)$ & 0.19 \\
\hline Severe comorbidity & $1161(81.5 \%)$ & $264(18.5 \%)$ & $1.5(1.3-1.8)$ & $<0.0001$ \\
\hline Total & $522(36.6 \%)$ & $903(63.4 \%)$ & $1.7(1.5-1.9)$ & $<0.0001$ \\
\hline \multicolumn{5}{|c|}{ ALCYONE study with Daratumumab } \\
\hline Criteria & Fulfill IC & No fulfillment of IC & HR $(95 \%$ CI $)$ & $P$ \\
\hline Hemoglobulin $<5$ & $1315(93.5 \%)$ & $92(6.5 \%)$ & $1.2(0.9-1.5)$ & 0.13 \\
\hline COPD/Asthma & $1299(91.2 \%)$ & $126(8.8 \%)$ & $1.5(1.2-1.8)$ & $<0.0001$ \\
\hline Total & $476(33.8 \%)$ & $931(66.2 \%)$ & $1.7(1.5-1.9)$ & $<0.0001$ \\
\hline \multicolumn{5}{|l|}{$\leq 65(N=764)$} \\
\hline \multicolumn{5}{|c|}{ STaMINA, IFM2013-04, IFM DFCI 2009, EMN02 trials } \\
\hline Criteria & Fulfill IC & No fulfillment of IC & HR $(95 \%$ CI $)$ & $\boldsymbol{P}$ \\
\hline CRAB & $714(93.5 \%)$ & $50(6.5 \%)$ & $0.6(0.4-1.0)$ & 0.034 \\
\hline Measurable disease & $644(94.6 \%)$ & $37(5.4 \%)$ & $1.0(0.7-1.6)$ & 0.85 \\
\hline $\mathrm{PLC}<10 \%$ & $644(86.9 \%)$ & $97(13.1 \%)$ & $0.8(0.6-1.0)$ & 0.085 \\
\hline Kidney failure & $591(77.8 \%)$ & $169(22.2 \%)$ & $1.8(1.4-2.2)$ & $<0.0001$ \\
\hline $\mathrm{PS}>2$ & $651(85.2 \%)$ & $113(14.8 \%)$ & $1.8(1.4-2.2)$ & $<0.0001$ \\
\hline Amyloidosis & $740(96.9 \%)$ & $24(3.1 \%)$ & $2.3(1.4-3.6)$ & 0.0007 \\
\hline AMI within 6 mo. & $761(99.6 \%)$ & $3(0.4 \%)$ & $0.6(0.1-4.4)$ & 0.64 \\
\hline HIV & $764(100.0 \%)$ & $0(0.0 \%)$ & - & - \\
\hline Hepatitis & $764(100.0 \%)$ & $0(0.0 \%)$ & - & - \\
\hline Cancer within $5 \mathrm{Y}$ & $720(94.2 \%)$ & $44(5.8 \%)$ & $0.9(0.6-1.4)$ & 0.71 \\
\hline Severe comorbidity & $694(90.8 \%)$ & $70(9.2 \%)$ & $1.8(1.3-2.4)$ & $<0.0001$ \\
\hline Total & $345(45.2 \%)$ & $419(54.8 \%)$ & $1.3(1.0-1.5)$ & 0.021 \\
\hline
\end{tabular}

Table 2 shows distribution and effect on OS of single inclusion and exclusion criteria of clinical trials. HR are calculated using a Cox proportional hazard model.

Patients were divided into elderly patients ( $>65$ years) and younger patients ( $\leq 65$ years) and further separated into two cohorts, those that were eligible for protocol inclusion (IC) and those that were not (non-IC).

Patients were described by frequency and percentages for categorical variables and median and inter-quartile range (IQR) for continuous variables. Comparisons of categorical variables between groups were done by chisquare test and continuous variables were compared by Mann-Whitney $U$-test.
The Kaplan-Meier method was used for drawing survival curves and calculating survival probability at specific time points. The Cox proportional hazard method was used for calculating hazard ratios (HR) with $95 \%$ confidence intervals (CI) and $P$ values. The proportional hazard assumption was inspected by scaled Schoenfeld residuals. Sensitivity analyses were performed to investigate whether handling of missing values and definition of age cohorts could affect main findings this study. All $P$-values were two-sided and a $P$-value below 0.05 was considered 

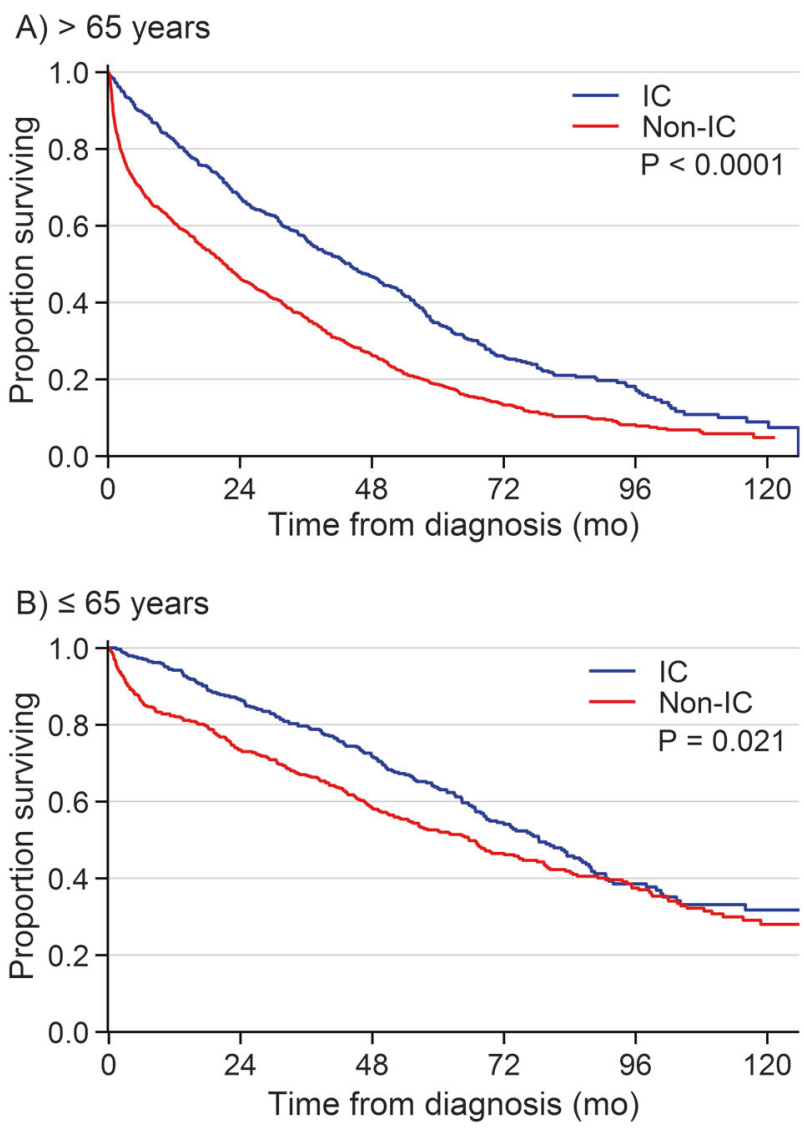

Fig. 1 Kaplan-Meier estimates of overall survival (OS) in the $>65$ years cohort (a) and the $\leq 65$ years cohort (b). The red lines represent patients that do not fulfill inclusion criteria (non-IC) and the blue lines show patients that do fulfill inclusion criteria (IC). For both age cohorts a significant difference was found

statistical significant. R statistical software version 3.3.3 (R Foundation for Statistical Computing, Vienna, Austria) was used for all calculations.

The characteristics and treatment of 1425 patients $>65$ years and 764 patients $\leq 65$ years are presented in Supplementary Table S4. Median follow-up for OS was $62.2 \mathrm{mo}$. in the elderly cohort and $77.0 \mathrm{mo}$. in the younger cohort.

In the elderly cohort $36.6 \%$ patients fulfilled the IC. The most frequent non-IC was kidney failure $(26.1 \%)$, severe comorbidity (18.5\%), and PS $>2(16.4 \%)$ (Table 1$)$. The non-IC cohort had a higher age, a higher ratio of men, a higher proportion of IgA MM, a higher level of $\beta 2$-microglobulin, LDH, and CRP, and lower levels of serum albumin. In the IC cohort more patients received melphalan and prednisone compared to the non-IC cohort $(65.5 \%$ vs. $47.8 \%, P<0.0001$ ) (Supplementary Table S5). Median OS in the non-IC cohort was OS 21.3 mo. compared to 44.0 mo. in the IC cohort, HR $=1.7$ (95\% CI: 1.5-1.9), $P<$ 0.0001 (Fig. 1a). OS depending on individual criteria is shown in Table 1 . Among patients $>65$ years $87(6.3 \%)$ received HDM-ASCT and of these $44(8.4 \%)$ were in the IC cohort for the elderly. Excluding these 87 patients from the calculations did not change the conclusion (OS in the nonIC cohort HR: 1.7 (95\% CI: $1.5-1.9), P<0.0001$ ).

In the younger patients $45.2 \%$ fulfilled IC. The most frequent non-IC was kidney failure (22.2\%), PS > $2(14.8 \%)$ and PLC $<10 \%(13.1 \%)$. The non-IC cohort had higher age, lower proportion of IgG MM, higher levels of $\beta 2$-microglobulin, LDH, and CRP. More patients in the IC cohort received cyclophosphamide and dexamethasone induction (52.0\% vs. $42.7 \%, P=0.010$ ). Median OS in the non-IC cohort was 65.5 mo. vs. 78.4 mo. in the IC cohort, HR: 1.3 (95\% CI: $1.0-1.5$ ), $P=0.021$ (Fig. 1b). In the $\leq 65$ years cohort $72.5 \%$ of the patients received HDM-ASCT. In the IC cohort $84.0 \%$ patients received HDM-ASCT compared to $62.9 \%$ in the non-IC cohort OR: 3.2 (95\% CI: $2.2-4.5$ ), $P<0.0001$. Single exclusion criteria associated with no HDM-ASCT are presented in Supplementary Table S6. When we compared OS for non-IC with those fulfilling IC in 547 patients treated with HDM-ASCT, we found no difference in OS, HR: 0.9 (95\% CI: $0.7-1.1), P=0.28$ (Supplementary Figure S1). Supplementary Table S7 illustrates the frequency and risk for not fulfilling the criteria for a phase III RCT in patients treated with HDM-ASCT. Kidney failure was the only criteria significantly associated with shorter OS with a HR of 1.5 (95\% CI: 1.1-2.0).

In this large population-based study our RWD show that only $36.6 \%$ of the elderly and $45.2 \%$ of younger patients fulfilled the inclusion and exclusion criteria for the eight chosen phase $3 \mathrm{RCT}$ and these patients have better OS. Drug registration phase III RCT are often the quickest and easiest design to show superiority of new treatment but not reflective of clinical standard of care in the community where we adjust the dose of the individual drugs according to the co-morbidity, frailty, and disease complications.

Renal failure, affected PS and severe co-morbidity were the most frequent exclusion criteria. In our real-world setting many younger patients with kidney failure and poor PS were treated with a transplant within 4-5 mo. from diagnosis and no difference was found in OS compared to patients fulfilling the IC. The EMN002 study, accepted a creatinine clearance $\leq 15 \mathrm{ml} / \mathrm{min}$ and in this study $53.3 \%$ patients fulfilled IC. No significant effect was found on outcome for the exclusion criteria "cancer within 5 years". We know that comorbidity affects OS but not if this inferior OS is caused by a less effective treatment [2].

Decision on inclusion in RCT is based on available data and this study is based on retrospective data collected for other purposes. However, annotation of all patients in the DMMR is completed yearly. Patients fulfilling IC in this study may have been included in RCT and thus have better OS due to new treatment strategies whereas we expect few in our non-IC to have been included in RCT. In our DMMR we annotate whether patients participate in clinical trials, but not which trials. From our DMMR we know that $10 \%$ 
of the elderly in the IC were included in clinical trials and $5 \%$ of the patients in the non-IC cohort. Among the younger patients $20 \%$ in the IC cohort were included in clinical trials and $15 \%$ in the non-IC cohort. The clinical phase III RCTs were the pamidronate trial, which do not affect OS [15] and the Hovon 95/EMN002 trial [12] and the Hovon 87 trial [3]

We plan to use the RWD from our DMMR and the DNPR to analyze outcome and major side effects of new drugs in patients in clinical practice with focus on patients that do not fulfill the inclusion criteria in RCT. We suggest that inclusion criteria in RCT for PS, renal disease, and other cancers are adjusted and that the design of studies is reconsidered to better reflect daily practice. Furthermore, we recommend word-wide investigator-initiated studies on patient's not fulfilling IC.

Acknowledgements The work was funded by The Research Fund at Rigshospitalet, Copenhagen.

Author contributions TWK and AJV conceived and designed the work, interpreted the results, drafted the manuscript and approved the final version. HG, NA, NFA, UCF, PG, and $\mathrm{CH}$ interpreted the results, revised the manuscript and approved the final version.

\section{Compliance with ethical standards}

Conflict of interest The authors declare that they have no conflict of interest.

\section{References}

1. Gimsing P, Holmström MO, Wirenfelt Klausen T, Frost Andersen N, Gregersen H, Pedersen RS, et al. The Danish national multiple myeloma registry. Clin Epidemiol. 2016;8:583-7.

2. Gregersen H, Vangsted AJ, Abildgaard N, Andersen NF, Pedersen RS, Frolund UC, et al. The impact of comorbidity on mortality in multiple myeloma: a Danish nationwide population-based study. Cancer Med. 2017;6:1807-16.

3. Zweegman S, van der Holt B, Mellqvist U-H, Salomo M, Bos GMJ, Levin M-D, et al. Melphalan, prednisone, and lenalidomide versus melphalan, prednisone, and thalidomide in untreated multiple myeloma. Blood. 2016;127:1109-16.

4. Abildgaard N, Vangsted A, Gregersen H, Andersen N, Pedersen $\mathrm{R}$, Plesner $\mathrm{T}$, et al. Continued improvement in overall survival in elderly multiple myeloma patients after 2008; a population based study from the Danish Multiple Myeloma Registry. Clin Lymphoma, Myeloma Leuk. 2015;15:e189.

5. Sonneveld P, Goldschmidt H, Rosiñol L, Bladé J, Lahuerta JJ, Cavo M, et al. Bortezomib-based versus nonbortezomib-based induction treatment before autologous stem-cell transplantation in patients with previously untreated multiple myeloma: a metaanalysis of Phase III Randomized, Controlled Trials. J Clin Oncol. 2013;31:3279-87.

6. San Miguel JF, Schlag R, Khuageva NK, Dimopoulos Ma, Shpilberg O, Kropff M, et al. Bortezomib plus melphalan and prednisone for initial treatment of multiple myeloma. N Engl J Med. 2008;359:906-17.

7. Bahlis NJ, Corso A, Mugge LO, Shen ZX, Desjardins P, Stoppa $\mathrm{AM}$, et al. Benefit of continuous treatment for responders with newly diagnosed multiple myeloma in the randomized FIRST trial. Leukemia. 2017;31:2435-42.

8. Durie BGM, Hoering A, Abidi MH, Rajkumar SV, Epstein J, Kahanic SP, et al. Bortezomib with lenalidomide and dexamethasone versus lenalidomide and dexamethasone alone in patients with newly diagnosed myeloma without intent for immediate autologous stem-cell transplant (SWOG S0777): a randomised, open-label, phase 3 trial. Lancet. 2017;389:519-27.

9. Stadtmauer EA, Pasquini MC, Blackwell B, Knust K, Bashey A, Devine SM, et al. Comparison of autologous hematopoietic cell transplant (autoHCT), bortezomib, lenalidomide (Len) and dexamethasone (RVD) consolidation with Len Maintenance (ACM), Tandem Autohct with Len Maintenance (TAM) and Autohct with Len Maintenance (AM) for up-Fron\{. Blood. 2016;128:LBA-1-LBA-1.

10. Moreau P, Hulin C, Macro M, Caillot D, Chaleteix C, Roussel M, et al. VTD is superior to VCD prior to intensive therapy in multiple myeloma: results of the prospective IFM2013-04 trial. Blood. 2016;127:2569-74.

11. Attal M, Lauwers-Cances V, Hulin C, Leleu X, Caillot D, Escoffre $M$, et al. Lenalidomide, bortezomib, and dexamethasone with transplantation for myeloma. N Engl J Med. 2017;376:1311-20.

12. Cavo M, Beksac M, Dimopoulos MA, Pantani L, Gay F, Hájek R, et al. Intensification therapy with bortezomib-melphalanprednisone versus autologous stem cell transplantation for newly diagnosed multiple myeloma: an Intergroup, Multicenter, Phase III Study of the European Myeloma Network (EMN02/HO95 MM Trial). Blood. 2016;128:673.

13. Vangsted AJ, Klausen TW, Andersen NF, Abildgaard N, Gang $\mathrm{AO}$, Gregersen $\mathrm{H}$, et al. Improved survival of multiple myeloma patients with late relapse after high-dose treatment and stem cell support, a population-based study of 348 patients in Denmark in 1994-2004. Eur J Haematol. 2010;85:209-16.

14. Helm-Petersen S, Sørrig R, Klausen TW, Preiss B, Frølund UC, Helleberg C, et al. Early relapsed disease of multiple myeloma following up-front HDM-ASCT: a study based on the Danish Multiple Myeloma Registry in the period 2005 to 2014. Leukemia. 2018;32:2054-2057.

15. Gimsing P, Carlson K, Turesson I, Fayers P, Waage A, Vangsted A, et al. Effect of pamidronate $30 \mathrm{mg}$ versus $90 \mathrm{mg}$ on physical function in patients with newly diagnosed multiple myeloma (Nordic Myeloma Study Group): a double-blind, randomised controlled trial. Lancet Oncol. 2010;11:973-82. 
Leukemia (2019) 33:550-553

https://doi.org/10.1038/s41375-018-0273-z

Acute lymphoblastic leukemia

\title{
$\mathrm{DUX4}_{\mathrm{HD2} 2}-\mathrm{DNA}_{\mathrm{ERG}}$ structure reveals new insight into DUX4- Responsive-Element
}

\author{
Xue Dong $^{1} \cdot$ Hao Zhang ${ }^{1} \cdot$ Nuo Cheng ${ }^{1} \cdot$ Kening Li $^{1} \cdot$ Guoyu Meng $\mathbb{D}^{1}$ \\ Received: 12 July 2018 / Revised: 23 August 2018 / Accepted: 31 August 2018 / Published online: 12 October 2018 \\ (c) The Author(s) 2018. This article is published with open access
}

Recently, we have reported a $2.6 \AA$ crystal structure of DUX $4_{\mathrm{HD} 2}$ complexed with a consensus $\mathrm{DRE}_{\text {consensus }}$ derived from the wild type DUX4 ChIP-seq analysis $[1,2]$ The $\mathrm{DRE}_{\text {consensus }}$ site is also present in the leukemia NALM6 and Reh cells harboring oncogenic DUX4/IGHs (Fig. 1a and Supplementary Figure 1a) [3, 4]. Furthermore, the GATXXGAT-like, TGAT-ATTA-like repeats are also frequently associated with wild type DUX4 and DUX4/IGH target genes (Fig. 1a). In order to gain more insight into the true nature of DUX4-DRE interaction, we have determined the structure of DUX4 HD2 bound with endogeneous ERG DNA sequences derived from the B-ALL patient RNA-seq and ChIP-seq analysis [3, 4].

The recombinant DUX4 HD2 domain was purified as described before [1]. The crystal of HD2-DRE $E_{E R G}$ diffracted remarkably well $(1.6 \AA)$ compared with that of $\mathrm{DUX} 4_{\mathrm{HD} 2}-$ $\mathrm{DRE}_{\text {consensus }}(2.6 \AA)$. The statistics detail of X-ray data collection is shown in Table 1. For structural determination, the refined HD2 structures (PDB codes: 5Z2S and 5Z2T) [1], but not DNA coordinates, were used for molecular replacement (MR) approach implemented in PHASER [5]. The DNA duplex of 5'-TGATGAGATTA-3'/3'ACTACTCTAAT-5' were built manually using COOT [5],

These authors contributed equally: Xue Dong, Hao Zhang, Nuo Cheng

Senior author: Guoyu Meng

Electronic supplementary material The online version of this article (https://doi.org/10.1038/s41375-018-0273-z) contains supplementary material, which is available to authorized users.

$\triangle$ Guoyu Meng

guoyumeng@shsmu.edu.cn

1 State Key Laboratory of Medical Genomics, Shanghai Institute of Hematology, Rui-Jin Hospital, Shanghai JiaoTong University School of Medicine and School of Life Sciences and Biotechnology, Shanghai JiaoTong University, 197 Ruijin Er Road, Shanghai 200025, China followed by TLS refinement using PHENIX.REFINE [6]. The final $R$ and $R_{\text {free }}$ factors are 20.1 and $20.7 \%$, respectively.

Consistent with previous report [1], one ERG DNA duplex can bind to two HD2 molecules (Fig. 1b). Unlike the previous $2.6 \AA \mathrm{HD} 2-\mathrm{DRE}_{\text {consensue }}$ structure, the electron density map of ERG DNA is of high quality (1.6 $\AA$, Supplementary Figure $1 b$ ) and allows clear registration of ERG sequences, $\quad 5^{\prime}-\mathrm{T}_{1} \mathrm{GATGAGATT}_{11}-3^{\prime} / 3^{\prime}-\mathrm{A}_{1} \mathrm{CTACTC}$ $\mathrm{TAA}_{11^{-}} 5^{\prime}$. The electron density map of the last pair of nucleotides, T11 and A11, are disordered and hence not available for model building. For the HD2 molecules, the final refined models contain residues Arg95 to Gln152. As reported before [1], the HD2 domain folds into a global domain of three helices, $\alpha 1-\alpha 3$, respectively. The $\mathrm{N}$ terminal poly-Arg/Lys motif, perpendicular to the helix $\alpha 1$, engages the DNA binding. In this structure, the Arg95 and Arg98 side-chains dip into the minor groove. In particular, Arg98 forms a hydrogen bond with the hydroxyl group of T1 nucleotide (Fig. 1c). Consistent with previous observation [1], the average $B$ factor of $\mathrm{R}_{95} \mathrm{RKR}_{98}$ in $\mathrm{DUX}_{\mathrm{HD}^{-}}$ $\mathrm{DNA}_{\text {ERG }}$ is $67.4 \AA^{2}$, much higher than the rest of the structure $\left(40.3 \AA^{2}\right.$ ), reiterating a secondary role in the twostep mechanism of DUX4-driven transactivation [1].

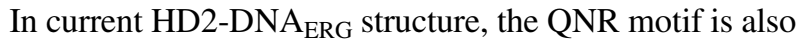
the major DNA-code-reading module (Figure 1d-f). The previous report suggests QNR can bind to the consensus TAAT repeat [1]. To our surprise, the Asn 144 and Arg 148 form two pairs of hydrogen bond with the G2 and A3 nucleotide (Fig. 1d). The invariant Asn144 among homeobox superfamily lies in the heart of the major groove. The carboxamide side-chain form two hydrogen bonds with the A3 nucleotide (2.7 and $3.0 \AA$, respectively). In parallel with Asn144 side chain lies the Arg148 guanidinium head group, which in turn forms two hydrogen with the G2 nucleotide (3.1 and $3.1 \AA$, respectively). Besides, in the region surrounding $\mathrm{T}_{1} \mathrm{GAT}_{4}$ nucleotides, it is enriched with positively charged residues including Arg95, Arg96, Lys97, Arg98, Arg137, Trp141, Arg145, Arg148 and His149 (Fig. 1e). Of note, the dual side-chain configuration observed in His 149 


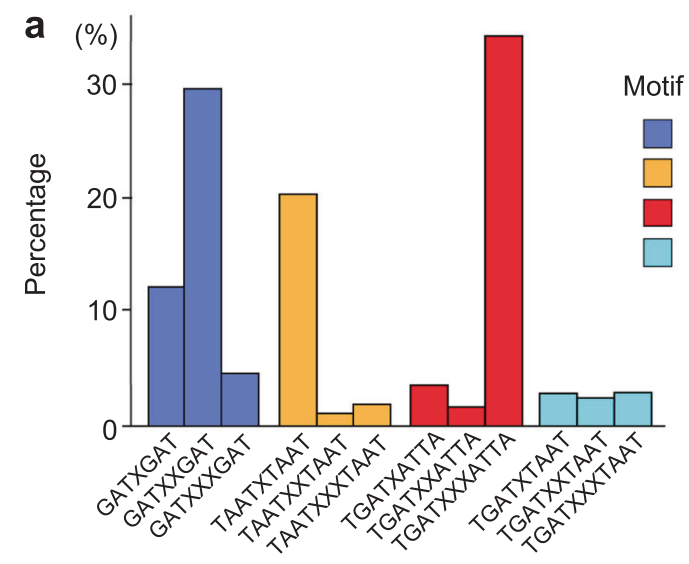

C

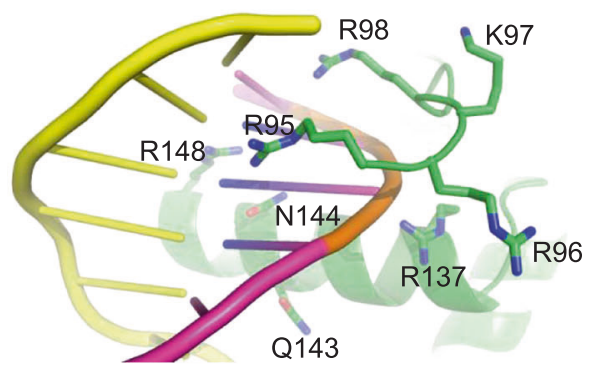

e
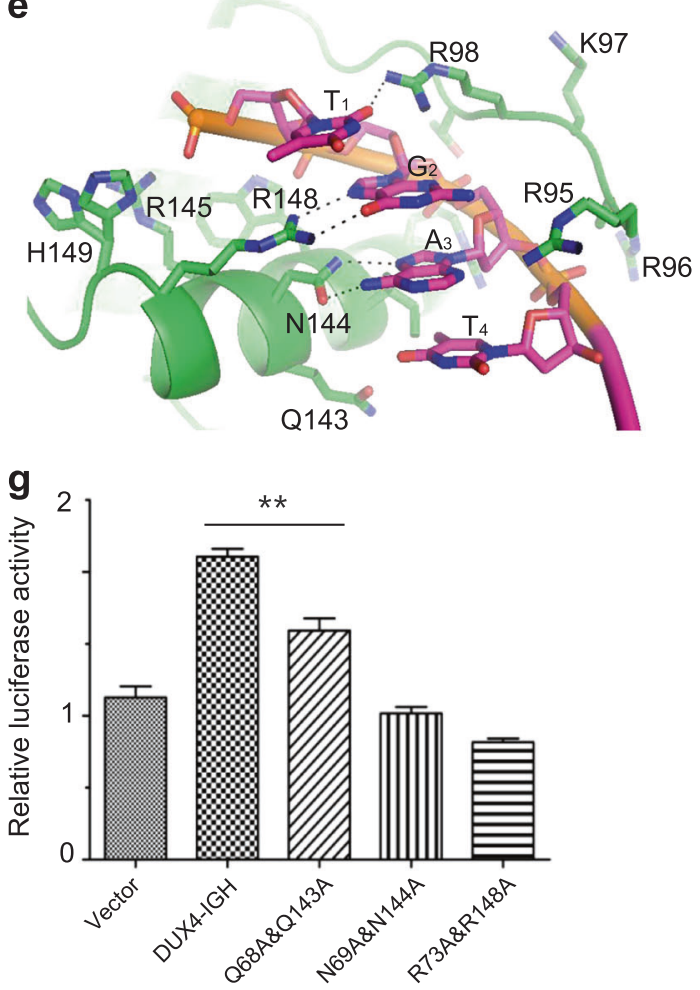

is a strong indication of side-chain reshuffle upon DNA binding/recognition. In the major groove also lies another important residues, Gln143. Consistently with previous observation, the high-resolution $\mathrm{DUX}_{\mathrm{HD} 2}-\mathrm{DNA} \mathrm{ERG}_{\mathrm{ER}}$ structure reveals an interesting Gln143-water-nucleotide network
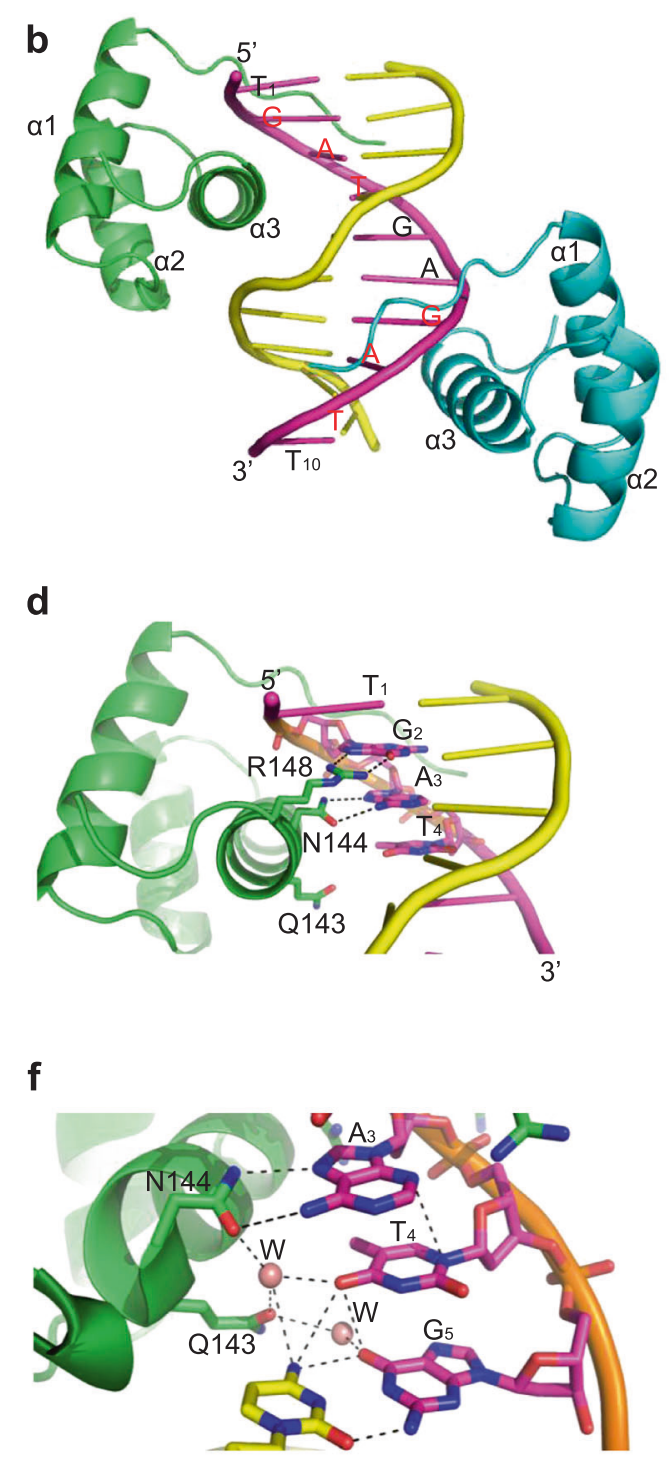

h

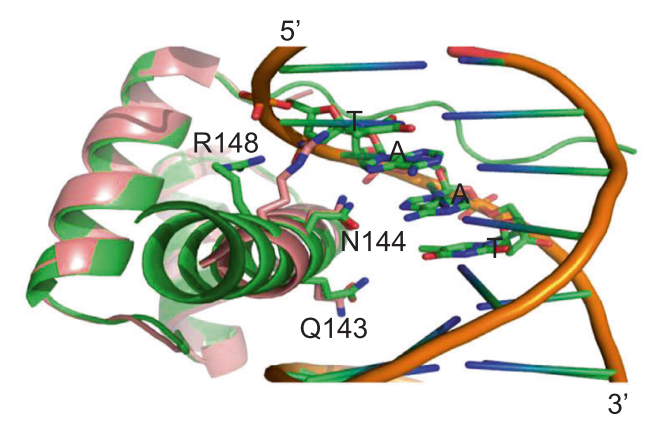

in the major groove (Fig. 1f). Furthermore, via watermediated hydrogen-bonding network, Gln143 can contribute to side-chain orientation of Asn144 and its subsequent reading/binding of A3 nucleotdie. 
Fig. 1 Structural and functional cauterization of DUX4-ResponsiveElement (DRE) sites. a Repetitive motifs in wild type DUX4 and DUX4/IGH target genes. The published DUX4 and DUX4/IGH ChIPseq datasets were used for this re-analysis. The percentage of different TGAT- and TAAT-like repeats in the DUX4 and DUX4/IGH target genes are shown in this figure. b ERG DNA duplex in complex with two HD2s. The ERG chains are colored with magenta and yellow. The $5^{\prime}$ and $3^{\prime}$ ends are labeled. The forward chain of $5^{\prime}-\mathrm{T}_{1}$ GATGAGATT $_{10} \mathbf{3}^{\prime}$ is labeled. The repetitive GAT sequences are highlighted in red. Of note, nucleotide A11 is disordered and absence in the final model. HD2 molecules are colored with green and cyan, respectively. c Enlarged view of minor groove engagement by poly-Arg/Lys motif. The protein side-chains are labeled and shown in stick representation. d Enlarged view of major groove interaction by QNR motif. The DNA nucleotide $\mathrm{T}_{1} \mathrm{GAT}_{4}$ (magenta) are labeled and shown in stick representation. The hydrogen bonds are shown in dashed lines. e Other DNA-binding residues around $\mathrm{T}_{1} \mathrm{GAT}_{4}$. A highly positive pocket delineated by residues Arg95, Arg96, Lys97, Arg98, Arg137, Trp141, Arg145, Arg 148 and His 149 are assembled upon DNA binding. f The water-mediated hydrogen network around Gln143. Water molecules observed in between Gln 143 and $\mathrm{T}_{4} \mathrm{G}_{5}$ nucleotide are shown in sphere representation. The hydrogen-bonding network are shown in dashed lines. $\mathbf{g}$ Luciferase assay using ERG site. The DRE site derived from $E R G$ gene [4] was used in this assay. The transcriptional activities of the wild type DUX4/IGH and the mutations that target the QNR motifs in HD1-HD2 were characterized by the standard luciferase reporter kit (Promega). The luciferase activities were presents as mean \pm SE. The statistical significance was assessed by the student's $t$ test analysis $(* * P<0.01)$. h Structural superimposition between DUX4 (pink) and PAX (green) HDs in the context of PAX-TAAT structrue (PDB code: 1FJL). The different QNR motifs are shown in stick representation. $\mathrm{T}_{1} \mathrm{AAT}_{4}$ are labeled

The importance of QNR motif in DUX4/IGH-driven transactivation was vigorously checked in our previous report [1]. Here, the major DNA-reading motif was characterized with luciferase assay using ERG DNA sequence. The perturbation of the DNA engaging reisidues Gln68/ Gln143, Asn69/Asn144 and Arg73/Arg148 significantly impaired the transcription activity of DUX4/IGH (Fig. 1g). In good agreement with our previous assays, the mutations of Asn69/Asn144 and Arg73/Arg148 were always more destructive when compared to that of Gln68/Gln143, reiterating the biological relevance of $\mathrm{HD} 2-\mathrm{DNA}_{\mathrm{ERG}}$ and HD2-DRE $E_{\text {consensus }}$ structures. Indeed, the Asn144, Arg148 and Gln143 residues/positions appear to be the most, relative less and least conserved positions, respectively (Supplementary Figure 2). This has led to the proposal that, while the invariant Asn residue in the middle of the DNAbinding-triol might play the most critical role in DNA binding, the residues Gln/Arg (or other variants) in the flanking positions might contribute to motif recognition specificity (Supplementary Figure 2).

Until now, it is well recognized that most HD domains can bind DNA with TAAT motif. Like PAX/PAX3, DUX4 HDs, which contain the same sets of poly-Arg/Lys and QNR motifs, can interact with TAAT. Agreeably, using luciferase assay, Zhang and co-workers demonstrated that TAAT-rich sequences were required for DUX4-driven
Table 1 Data collection and structure refinement statistics of DUX4 HD2 associated with ERG site

\begin{tabular}{|c|c|}
\hline Protein & $\mathrm{DUX}_{\mathrm{HD} 2}-\mathrm{DNA}_{\mathrm{ERG}}$ \\
\hline \multicolumn{2}{|l|}{ Data collection } \\
\hline Space group & $P 3_{1}$ \\
\hline \multicolumn{2}{|l|}{ Unit cell dimension $(\AA)$} \\
\hline a & 32.6 \\
\hline $\mathrm{b}$ & 32.6 \\
\hline $\mathrm{c}$ & 126.6 \\
\hline Molecule per ASU & $2 \mathrm{HD} 2,1$ double-chain $\mathrm{DNA}_{\mathrm{ERG}}$ \\
\hline Derivative & Native \\
\hline Source/Station ${ }^{\mathrm{a}}$ & BL19U \\
\hline Wavelength $(\AA)$ & 0.9785 \\
\hline Resolution range $(\AA)$ & $63.3-1.60$ \\
\hline Observations $(I / \sigma(I)>0)$ & 160629 \\
\hline Unique reflections $(I / \sigma(I)>0)$ & $19696(2803)$ \\
\hline High-resolution shell $(\AA)$ & $1.69-1.60$ \\
\hline$R_{\mathrm{sym}}(\%)^{\mathrm{b}, \mathrm{c}}$ & $7.2(91.2)$ \\
\hline$<I / \sigma(I)>^{\mathrm{c}}:$ & $11.4(1.8)$ \\
\hline Completeness $^{\mathrm{c}}(\%)$ : & $99.2(97.0)$ \\
\hline Redundancy ${ }^{\mathrm{c}}$ : & $8.2(6.8)$ \\
\hline $\mathrm{CC}_{1 / 2}$ & $0.99(0.76)$ \\
\hline \multicolumn{2}{|l|}{ Structure refinement } \\
\hline Resolution range $(\AA)$ & $28.2-1.60$ \\
\hline$R$-factor $(\%)$ & 20.1 \\
\hline$R$-factor (high-resolution shell) ${ }^{\mathrm{d}}$ & 28.3 \\
\hline$R_{\text {free }}(\%)^{\mathrm{e}}$ & 20.7 \\
\hline$R_{\text {free }}($ high-resolution shell) & 34.7 \\
\hline Total number of non-hydrogen atoms & 1492 \\
\hline Protein atoms & 1376 \\
\hline Water molecules & 116 \\
\hline \multicolumn{2}{|l|}{ R.m.s. deviations: ${ }^{f}$} \\
\hline Bond length $(\AA)$ & 0.013 \\
\hline Bond angle $\left({ }^{\circ}\right)$ & 1.227 \\
\hline Main chain $B$-factors $\left(\AA^{2}\right)$ & 3.676 \\
\hline Side-chain $B$-factors $\left(\AA^{2}\right)$ & 7.047 \\
\hline Wilson $B$-factor $\left(\AA^{2}\right)$ & 28.4 \\
\hline Average $B$-factor protein atoms $\left(\AA^{2}\right)$ & 40.3 \\
\hline \multicolumn{2}{|l|}{ Ramachandran statistics (\%) } \\
\hline Most favored region & 96.4 \\
\hline Allowed regions & 3.6 \\
\hline Outliers & 0 \\
\hline
\end{tabular}

${ }^{a}$ Beamline designations refer to the Shanghai Synchrotron Radiation Facility, Shanghai, P. R. of China

${ }^{\mathrm{b}} R_{\text {sym }}=\Sigma(I-<I>)^{2} / \Sigma I^{2}$

'Overall, high-resolution shell in parentheses

${ }^{\mathrm{d}}$ High-resolution shell: $1.6847-1.6000$

${ }^{\mathrm{e}} R_{\text {free }}$ calculated using $5 \%$ of total reflections omitted from refinement

${ }^{\mathrm{f}}$ R.m.s. deviations report root mean square deviations from ideal bond lengths/angles and of $B$-factors between bonded atoms [8]

transactivation [7]. Consistently, TAAT repeat was also frequently associated with leukemia cell lines that contain DUX4/IGH [3, 4] (Fig. 1a and Supplementary Figure 1a). 
In this report, the $1.6 \AA \mathrm{DUX}^{\mathrm{HD} 2}$-DNA $\mathrm{ERG}_{\mathrm{ERG}}$ structure demonstrates a strong association between DUX4 homeobox and TGAT. As discussed in our previous report [1], the coordinates and positioning of the second HD2 molecule in this structure also allow the envisage/modeling of DUX4 $_{\text {HD1-HD2 }}$ complexed with ERG site 5'-TGATGA GATTA-3', in which it also contains repetitive GAT sequences. This has led to the re-examination of the pre-

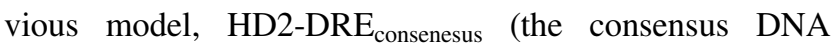
sequence used in previous crystallization is $5^{\prime}$-TTCTAA TCTAATCA-3' ${ }^{\prime} / 3^{\prime}$-AAGATTAGATTAGT-5'). When GAT repeat was modeled into the DNA electron density map that engages QNR interaction, the $R_{\text {free }}$ factor is $29.4 \%$ (previous refinement is 29.9\%). In light of the new HD2-DNA ${ }_{E R G}$ high-resolution structure, the previous DNA registration in the poor electron density map of HD2-DRE $\mathrm{E}_{\text {consensue }}\left(R_{\text {sym }}\right.$ and signal-to-noise level in the highest resolution shell 2.7$2.6 \AA$ is $155 \%$ and 1.5 , respectively) might be interpreted with caution although it suggests a TAAT-binding possibility. In this model, it is clear that DUX4 HD2 can bind to GATXXGAT-like repeat. In support of this claim, the revision of the published DUX4/IGH ChIP-seq data showed that, among 364 DUX4 target genes, 109 genes contain GATXXGAT motif (Fig. 1a). In addition, TAAT-like repeat is also frequently reported in wild type DUX4 and DUX4/IGH [3, 7] (Fig. 1a). Consistently, via structural superimposition and sequence alignment, a possible engagement between DUX4 HDs and TAAT site could be envisaged (Fig. 1h). Indeed, the TGAT motif (in the forward DNA chain) and the TAAT motif (in the complementary DNA chain) display the strongest consensus in DUX4-driven transactivation (Supplementary Figure 1a). Taken together, the new DUX4 $4_{\mathrm{HD} 2}-\mathrm{DNA}_{\mathrm{ERG}}$ structure shed new insight into the DUX4-DRE recognition: (1) DUX4 HD1-HD2 might bind preferentially to the repetitive DNA sequences containing 5'-GATXXGAT-3', 5'-TAATX TAAT- $3^{\prime}$ and 5-TGAT/TAAT-5' (the complementary chain is underlined) motifs. (2) This helps to define a novel HD subclass, in which DUX double homeobox can display remarkable double-kiss and double tolerance activities in recognition of TGAT- and TAAT-like repetitive sequences. In addition, the current observation/proposal in DUX4 will undoubtedly prompts a more extensive revision of the versatile HD-DNA interactions summarized in Supplmentary Figure 2.

Acknowledgements This work was supported by research grants 81770142 from National Natural Scientific Foundation of China (to GM), a research grant 20152504 from "Shanghai Municipal Education Commission-Gaofeng Clinical Medicine Grant Support" (to GM), "The Program for Professor of Special Appointment (Eastern Scholar) at Shanghai Institute of Higher Learning" (to GM). We thank the personnel of beamlines BL17U/18U1/19U1 (SSRF/NFPS, Shanghai, China) for help during data collection. We also thank colleagues in the DUX4 field for their critical comments to our previous report, which has led to the re-investigation of DUX4-DRE $E_{\mathrm{ERG}}$ and the discovery/ recognition of GAT repeat in DUX4-driven transactivation.

Author Contributions: Conceived and designed the experiments: GM. Performed the experiments: XD, HZ, NC, Analyzed the data: XD, HZ, NC, KL, GM. Preparation of figures manuscripts: XD, HZ, NC, GM. Wrote the paper: GM.

\section{Compliance with ethical standards}

Conflict of interest The authors declare that they have no conflict of interest.

Open Access This article is licensed under a Creative Commons Attribution 4.0 International License, which permits use, sharing, adaptation, distribution and reproduction in any medium or format, as long as you give appropriate credit to the original author(s) and the source, provide a link to the Creative Commons license, and indicate if changes were made. The images or other third party material in this article are included in the article's Creative Commons license, unless indicated otherwise in a credit line to the material. If material is not included in the article's Creative Commons license and your intended use is not permitted by statutory regulation or exceeds the permitted use, you will need to obtain permission directly from the copyright holder. To view a copy of this license, visit http://creativecommons. org/licenses/by/4.0/.

\section{References}

1. Dong X, Zhang W, Wu H, Huang J, Zhang M, Wang P, et al. Structural basis of DUX4/IGH-driven transactivation. Leukemia. 2018;32:1466-76.

2. Geng LN, Yao Z, Snider L, Fong AP, Cech JN, Young JM, et al. DUX4 activates germline genes, retroelements, and immune mediators: implications for facioscapulohumeral dystrophy. Dev Cell. 2012;22:38-51.

3. Tanaka Y, Kawazu M, Yasuda T, Tamura M, Hayakawa F, Kojima $\mathrm{S}$, et al. Transcriptional activities of DUX4 fusions in B-cell acute lymphoblastic leukemia. Haematologica. 2018, pii: haematol. 2017.183152.

4. Zhang J, McCastlain K, Yoshihara H, Xu B, Chang Y, Churchman ML, et al. Deregulation of DUX4 and ERG in acute lymphoblastic leukemia. Nat Genet. 2016;48:1481-9.

5. CCP4. The CCP4 suite: programs for protein crystallography. Acta Crystallogr. 1994;50:760-3.

6. Adams PD, Afonine PV, Bunkóczi G, Chen VB, Davis IW, Echols $\mathrm{N}$, et al. PHENIX: a comprehensive Python-based system for macromolecular structure solution. Acta Crystallogr D Biol Crystallogr. 2010;66:213-21.

7. Zhang Y, Lee JK, Toso EA, Lee JS, Choi SH, Slattery M, et al. DNA-binding sequence specificity of DUX4. Skelet Muscle. 2016;6:8.

8. Engh RA, Huber R. Accurate bond and angle parameters for X-ray protein structure refinement. Acta Crystallogr A. 1991;47: $392-400$ 
Leukemia (2019) 33:554-558

https://doi.org/10.1038/s41375-018-0274-y

Acute myeloid leukemia

\title{
Second cycle remission achievement with $7+3$ and survival in adults with newly diagnosed acute myeloid leukemia: analysis of recent SWOG trials
}

\author{
Megan Othus ${ }^{1}$ - Elihu H. Estey ${ }^{2,3}$ - Guillermo Garcia-Manero ${ }^{4}$ Brent L. Wood ${ }^{5}$ - Derek L. Stirewalt ${ }^{2,6}$. \\ John E. Godwin ${ }^{7}$ James K. Weick ${ }^{8} \cdot$ Jeanne E. Anderson ${ }^{9} \cdot$ Frederick R. Appelbaum $^{2,6} \cdot$ Harry P. Erba $^{10,12}$. \\ Roland B. Walter $\mathbb{D}^{2,3,11}$
}

Received: 20 August 2018 / Accepted: 31 August 2018 / Published online: 12 October 2018

c) Springer Nature Limited 2018

Intensive chemotherapy will induce a complete morphologic remission (CR) in many persons with acute myeloid leukemia (AML). Even with continued treatment, however, relapse is common [1,2]. Many pretreatment factors, most notably cytogenetic and molecular profiles, are associated with disease recurrence and shorter survival and allow outcome prediction, albeit with limited accuracy, at the individual patient level [3-5]. It is increasingly recognized that post-treatment information provides valuable insight into which remissions are of higher quality. One important characteristic of more durable remissions is the lack of measurable residual disease (MRD) [6]. On the other hand, whether it matters that a remission is obtained early, i.e., with the first cycle of chemotherapy, has remained controversial. Data from historic and contemporary trials with double induction chemotherapies showed patients who achieved a CR with the first induction cycle were less likely to relapse than those requiring two courses of therapy to

Electronic supplementary material The online version of this article (https://doi.org/10.1038/s41375-018-0274-y) contains supplementary material, which is available to authorized users.

Megan Othus

mothus@ fredhutch.org

1 Public Health Division and SWOG Statistical Center, Fred Hutchinson Cancer Research Center, Seattle, WA, USA

2 Clinical Research Division, Fred Hutchinson Cancer Research Center, Seattle, WA, USA

3 Department of Medicine, Division of Hematology, University of Washington, Seattle, WA, USA

4 Department of Leukemia, MD Anderson Cancer Center, Houston, TX, USA

5 Department of Laboratory Medicine, Division of enter CR [7, 8]. Contrasting these findings, an analysis of six ECOG (now ECOG-ACRIN) trials conducted between 1983 and 1993 indicated patients who achieved a CR after one or two cycles of induction chemotherapy had similar prognoses [9]. The relationship between timing of remission achievement and outcome has not been examined in contemporary cohorts of adults treated with $7+3$. Here, we used data from participants of five SWOG trials between 1983 and 2015 and studied the association between prognosis and need for $7+3$ reinduction therapy and how it has changed over time.

We analyzed 1247 patients randomized to $7+3$ in treatment arms of five National Cancer Institute (NCI)/National Clinical Trials Network (NCTN) trials conducted by SWOG. We analyzed patients aged 65 and younger: S8600 [10] $(n=530)$, S9031 [11] $(n=98)$, S9333 [12] $(n=57)$, S0106 [13] $(n=301)$, and S1203 [14] $(n=261)$. S8600 enrolled patients between November 1986 and December 1991, S9031 between November 1991 and January 1995, S9333 between January 1995 and December 1998, S0106 between May 2004 and August 2009, and S1203 between December 2012 and November 2015. While all studies

Hematopathology, University of Washington, Seattle, WA, USA

6 Department of Medicine, Division of Medical Oncology, University of Washington, Seattle, WA, USA

7 Providence Cancer Center Oncology and Hematology Care Clinic, Portland, OR, USA

8 VA Medical Center, North Palm Beach, FL, USA

9 Katmai Oncology Group, Anchorage, AK, USA

10 University of Alabama at Birmingham, Birmingham, AL, USA

11 Department of Epidemiology, University of Washington, Seattle, WA, USA

12 Present address: Duke University, Durham, NC, USA 
Table 1 Characteristics of study cohort

\begin{tabular}{|c|c|c|c|c|c|}
\hline & $\begin{array}{l}\mathrm{S} 8600 \\
(n=530)\end{array}$ & $\begin{array}{l}\text { S9031/S9333 } \\
(n=156)\end{array}$ & $\begin{array}{l}\text { S0106 } \\
(n=301)\end{array}$ & $\begin{array}{l}\mathrm{S} 1203 \\
(n=261)\end{array}$ & $P$ value \\
\hline Median age, years (range) & $45(15-64)$ & $61(56-65)$ & $48(18-60)$ & $48(19-60)$ & $<0.001$ \\
\hline Gender, $n(\%)$ & & & & & 0.68 \\
\hline Female & $247(47)$ & $70(45)$ & $147(49)$ & $131(50)$ & \\
\hline Male & $283(53)$ & $86(55)$ & $154(51)$ & $130(50)$ & \\
\hline Performance status, $n(\%)$ & & & & & $<0.001$ \\
\hline $0-1$ & $374(73)$ & $114(75)$ & $255(85)$ & $221(85)$ & \\
\hline $2-4$ & $140(27)$ & $38(25)$ & $44(15)$ & $40(15)$ & \\
\hline Cytogenetic risk, $n(\%)$ & & & & & 0.0035 \\
\hline Favorable & $9(9)$ & $5(5)$ & $42(19)$ & $28(11)$ & \\
\hline Intermediate & $56(58)$ & $68(62)$ & $126(57)$ & $168(66)$ & \\
\hline Unfavorable & $32(33)$ & $36(33)$ & $55(25)$ & $60(23)$ & \\
\hline Missing & 97 & 109 & 223 & 256 & \\
\hline NPM1, FLT3/ITD status, $n(\%)$ & N/A & N/A & & & 0.76 \\
\hline$N P M 1-$ and/or $F L T 3 / I T D+$ & & & $153(84)$ & $144(85)$ & \\
\hline$N P M 1+$ and FLT3- & & & $30(16)$ & $25(15)$ & \\
\hline NPM1, FLT3/ITD unknown & & & 183 & 169 & \\
\hline Type of AML, $n(\%)$ & & & & & $<0.001$ \\
\hline De novo & $506(95)$ & $122(78)$ & $301(100)$ & $236(90)$ & \\
\hline Secondary & $24(5)$ & $34(22)$ & $0(0)$ & $25(10)$ & \\
\hline \multicolumn{6}{|l|}{ Laboratory studies at baseline } \\
\hline WBC $\left(\times 10^{3} / \mu \mathrm{L}\right)$, median (range) & $18(0-416)$ & $13(1-274)$ & $12(0-244)$ & $13(1-800)$ & 0.029 \\
\hline Platelets $\left(\times 10^{3} / \mu \mathrm{L}\right)$ & $54(2-700)$ & $49(6-1,200)$ & $55(7-9,300)$ & $50(4-8,500)$ & 0.045 \\
\hline Marrow blasts $(\%)$ & $74(0-99)$ & $61(10-99)$ & $66(3-100)$ & $60(0-100)$ & $<0.001$ \\
\hline Number of induction courses, $n(\%)$ & & & & & 0.036 \\
\hline 1 & $381(72)$ & $126(82)$ & $234(78)$ & $198(76)$ & \\
\hline 2 & $149(28)$ & $27(18)$ & $66(22)$ & $63(24)$ & \\
\hline Induction response, $n(\%)$ & & & & & $<0.001$ \\
\hline $\mathrm{CR}$ on first induction & $219(41)$ & $74(48)$ & $178(59)$ & $137(52)$ & \\
\hline $\mathrm{CR}$ on reinduction & $68(13)$ & $7(5)$ & $32(11)$ & $27(10)$ & \\
\hline No CR & $243(46)$ & $72(47)$ & $91(30)$ & 97 (37) & \\
\hline
\end{tabular}

were limited to patients with newly diagnosed AML, the exact eligibility criteria differed between these trials. For example, trials S9031 and S9333 were restricted to patients aged 55 and older; the other studies included patients aged 18 and older. Patients with secondary AML were not eligible for S0106, which tested the addition of the CD33 antibody-drug conjugate gemtuzumab ozogamicin to induction chemotherapy. There were also differences in the chemotherapy regimens administered since, in all five trials, $7+3$ was given as per standard at that time. Specifically, in S8600, S9031, and S9333, the cytarabine and daunorubicin doses were $200 \mathrm{mg} / \mathrm{m}^{2}$ and $45 \mathrm{mg} / \mathrm{m}^{2}$, respectively, In S0106, doses used were $100 \mathrm{mg} / \mathrm{m}^{2}$ and $60 \mathrm{mg} / \mathrm{m}^{2}$, respectively. Finally, in S1203, doses of $100 \mathrm{mg} / \mathrm{m}^{2}$ for cytarabine and $90 \mathrm{mg} / \mathrm{m}^{2}$ for daunorubicin were used. Institutional review boards of participating institutions approved all protocols, and patients were treated according to the Declaration of Helsinki.

CR was defined conventionally ( $<5 \%$ marrow blasts by morphology, absolute neutrophil count $[\mathrm{ANC}] \geq 1000 / \mu \mathrm{L}$, platelets $\geq 100,000 / \mu \mathrm{L}$ ) [2]. Overall, survival (OS) was measured from the date of study registration/randomization to date of death due to any cause; patients last known to be alive were censored at the date of last contact. Relapsefree survival (RFS) was measured from the date of CR to the first of relapse from CR or death due to any cause; patients last known to be alive in CR were censored at the date of last contact. OS and RFS were estimated using the Kaplan-Meier method. Multivariable Cox regression models included covariates (modeled quantitatively unless otherwise specified): age at study registration, gender, cytogenetic risk, pre-study white blood cell counts (WBC), 

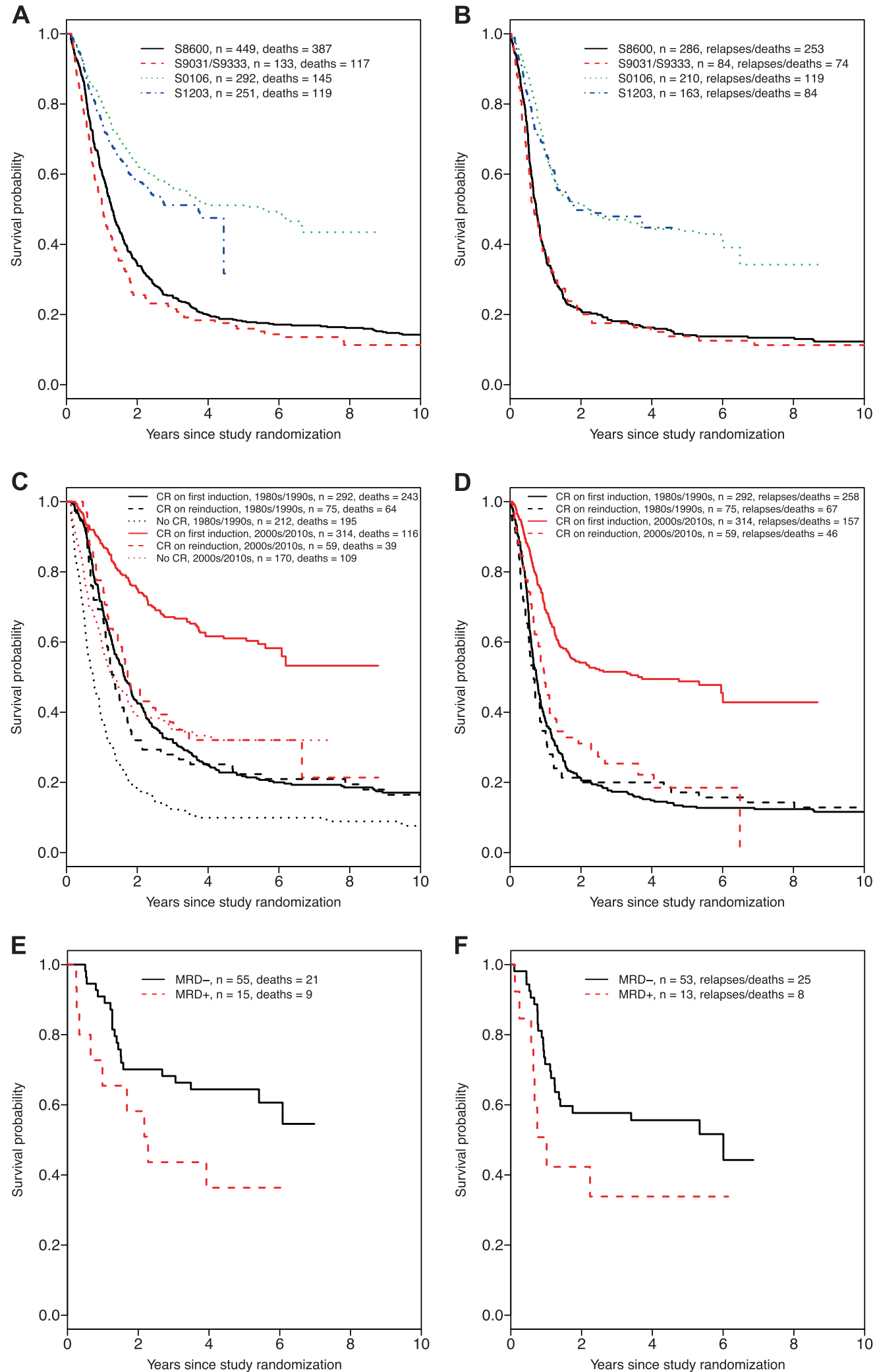

Fig. 1 Treatment outcomes in study cohort. a Overall survival and b relapse-free survival by trial/decade. c Overall survival and d relapse-free survival by CR status and decade of therapy (1980s/ 1990s versus 2000s/2010s). e Overall survival and f relapse-free

survival in patients treated on S0106 who achieved morphologic CR with the first cycle of $7+3$, stratified by results from MRD test, assessed centrally by multiparameter flow cytometry 
pre-study platelets, pre-study marrow blasts, type of AML (secondary vs. de novo), indicator of receiving reinduction and study/protocol. We analyzed study/protocol separately and also grouped the studies by 20-year period (S8600/ S9031/S9333 representing 1980s and 1990s vs. S0106/ S1203 representing 2000s and 2010s). The earlier of these groups (S8600/S9031/S9333) corresponds to the period of time covered in the analyses previously reported by ECOG-ACRIN [9]. NPMI and FLT3 mutation data were available for participants of S0106 and S1203 and separate regression models in restricted to these two trials were fit that included these molecular data. To control for the fact that patients needed to live long enough to receive reinduction, we performed landmark analyses among the patients who were alive at day 45 . However, to allow more direct comparison with the data previously reported by ECOG-ACRIN, which were not based on landmark analyses [9], we also performed analyses among all patients. All analyses were performed with R (http://www.rproject.org).

Table 1 summarizes the characteristics of the 1247 patients included in our analysis, group by decade of treatment (S8600 vs. S9031/S9333 vs. S0106 vs. S1203). Consistent with the differences in eligibility criteria noted above, patients enrolled in S9031/S9333 were older than those on the other studies. The proportion of patients with performance status 2 and higher decreased over time. In contrast, CR rates were higher in the studies conducted more recently compared to the earlier ones. In parallel with improved CR rates, OS and RFS were higher in S0106 and S1203 compared to S8600 and S9031/S9333 (Fig. 1a, b).

We then built multivariable models to examine the relationship between CR achievement with the second cycle of 7+3 and OS as well as RFS in the older cohort of studies (S8600, S9031, and S9333) as well as the newer cohort of studies (S0106 and S1203) separately. After adjustment for age, gender, performance status, cytogenetic risk, WBC, platelet count, percentage of blasts in the bone marrow, and type of AML, CR achievement only upon reinduction chemotherapy was not statistically significantly associated with OS (hazard ratio (HR) $=1.19$ [95\% confidence interval: $0.89-1.59$ ], $P=0.25$; Supplementary Table 1 ) or RFS $(\mathrm{HR}=1.15$ [0.86-1.54], $P=0.34$; Supplementary Table 2) in the older studies. These findings are similar to those reported by Rowe and colleagues on 1980 adults with newly diagnosed AML treated on six consecutive ECOG trials conducted in the 1980s and early 1990s [9]. In the ECOG trial analysis, 5- and 10-year DFS and OS was not significantly affected by the need for one cycle or two cycles of induction chemotherapy in any of the six trials, even after multivariable adjustment. Using the same methodology as the prior ECOG analysis (analyzing all patients instead of performing a landmark analysis), we found similar results to the landmark analyses above for both OS $(\mathrm{HR}=1.21[0.91-1.63], P=0.19)$ and RFS $(\mathrm{HR}=1.14$ [0.85-1.53], $P=0.37)$.

In contrast to the data obtained from the older studies, we found receiving two cycles of induction chemotherapy before $\mathrm{CR}$ is documented was associated with worse outcome in the S0106 and S1203 trials. Specifically, in these two trials, CR achievement only upon reinduction chemotherapy was statistically significantly associated with worse $\mathrm{OS} \quad(\mathrm{HR}=1.82$ [1.24-2.66], $P=0.002$; Supplementary Table 3$)$ or RFS (HR $=1.90$ [1.34-2.70], $P<0.001$; Supplementary Table 4). Fitting a model with an interaction between time-period and reinduction, that interaction was significant (for OS: $P=0.046$; for RFS: $P=0.016)$. Kaplan-Meier estimates of OS and RFS stratified by older vs. newer studies summarize these results graphically (Fig. 1c, d).

These findings indicate adults with newly diagnosed AML treated on more recent cooperative group trials who achieve remissions early, i.e., with the first cycle of $7+3$ chemotherapy, have better survival outlooks than those who need two cycles of chemotherapy to enter a CR, even after adjustment for other risk factors. These data are reminiscent of those obtained from trials of doubleinduction chemotherapy regimens [7, 8]. Why the relationship between time-of-remission achievement and outcome with $7+3$ therapy is not seen in older studies is unclear. Possibilities may include the change in anthracycline dose over time (i.e., chemotherapy resistance may be more apparent after the first cycle of chemotherapy with more intense regimens), selection of patients who receive on-study reinduction therapy, and changes in frequency/ routine of transplantation, among others.

Finally, having found evidence that early achievement of morphologic $\mathrm{CR}$ was associated with survival with $7+3$ chemotherapy, we examined whether data from MRD tests provide additional prognostic information in such patients. For S0106, we previously reported patients in remission without flow cytometric evidence of MRD after completion of induction chemotherapy (either 1 or 2 courses of $7+3$ ) had better outcomes than patients who achieved CR with up to two courses of chemotherapy but had a positive MRD test [15]. Here, we limited these analyses to patients who achieved a morphologic $\mathrm{CR}$ with the first cycle of $7+3$ and had data from MRD testing available $(n=70)$. Acknowledging limited power in these analyses because of a relatively small sample size, we found having a negative MRD test $(n=55)$ after cycle 1 was associated with statistically significantly better OS $(P=0.049)$ and a trend toward better RFS $(P=0.098)$ compared to having a positive MRD test after cycle 1 ( $n=15$; Fig. 1e, f). Patients who tested 
negative for MRD after the first cycle of $7+3$ also had significantly better RFS $(p=0.02)$ and a statistically nonsignificant trend $(p=0.11)$ toward better OS than those who achieved a MRD test-negative CR only after two therapy cycles (Supplementary Figure 1). Together, our findings not only highlight the prognostic significance of achieving a remission early, but also support the value of "MRD ${ }^{\text {neg }}$ CR" as recently proposed by the European LeukemiaNet [2] as new response entity.

Acknowledgements The authors gratefully acknowledge the important contributions of the late Dr. Stephen H. Petersdorf to SWOG and to study S0106. Research reported in this publication was supported by a grant from the National Cancer Institute/National Institutes of Health grants CA180888 and CA180819. RBW is a Leukemia \& Lymphoma Society Scholar in clinical research.

\section{Compliance with ethical standards}

Conflict of interest The authors declare that they have no conflict of interest.

\section{References}

1. Döhner H, Weisdorf DJ, Bloomfield CD. Acute myeloid leukemia. N Engl J Med. 2015;373:1136-52.

2. Döhner H, Estey E, Grimwade D, Amadori S, Appelbaum FR, Buchner T, et al. Diagnosis and management of AML in adults: 2017 ELN recommendations from an international expert panel. Blood. 2017;129:424-47.

3. Walter RB, Othus M, Burnett AK, Löwenberg B, Kantarjian HM, Ossenkoppele GJ, et al. Resistance prediction in AML: analysis of 4601 patients from MRC/NCRI, HOVON/SAKK, SWOG and MD Anderson Cancer Center. Leukemia. 2015;29:312-20.

4. Walter RB, Othus M, Paietta EM, Racevskis J, Fernandez HF, Lee JW, et al. Effect of genetic profiling on prediction of therapeutic resistance and survival in adult acute myeloid leukemia. Leukemia. 2015;29:2104-7.

5. Papaemmanuil E, Gerstung M, Bullinger L, Gaidzik VI, Paschka $\mathrm{P}$, Roberts ND, et al. Genomic classification and prognosis in acute myeloid leukemia. N Engl J Med. 2016;374:2209-21.

6. Hourigan CS, Gale RP, Gormley NJ, Ossenkoppele GJ, Walter RB. Measurable residual disease testing in acute myeloid leukaemia. Leukemia. 2017;31:1482-90.
7. Wheatley K, Burnett AK, Goldstone AH, Gray RG, Hann IM, Harrison CJ, et al. A simple, robust, validated and highly predictive index for the determination of risk-directed therapy in acute myeloid leukaemia derived from the MRC AML 10 trial. United Kingdom Medical Research Council's Adult and Childhood Leukaemia Working Parties. Br J Haematol. 1999; 107:69-79.

8. Terwijn M, van Putten WL, Kelder A, van der Velden VH, Brooimans RA, Pabst T, et al. High prognostic impact of flow cytometric minimal residual disease detection in acute myeloid leukemia: data from the HOVON/SAKK AML 42A study. J Clin Oncol. 2013;31:3889-97.

9. Rowe JM, Kim HT, Cassileth PA, Lazarus HM, Litzow MR, Wiernik $\mathrm{PH}$, et al. Adult patients with acute myeloid leukemia who achieve complete remission after 1 or 2 cycles of induction have a similar prognosis: a report on 1980 patients registered to 6 studies conducted by the Eastern Cooperative Oncology Group. Cancer. 2010;116:5012-21.

10. Weick JK, Kopecky KJ, Appelbaum FR, Head DR, Kingsbury LL, Balcerzak SP, et al. A randomized investigation of highdose versus standard-dose cytosine arabinoside with daunorubicin in patients with previously untreated acute myeloid leukemia: a Southwest Oncology Group study. Blood. 1996;88:2841-51.

11. Godwin JE, Kopecky KJ, Head DR, Willman CL, Leith CP, Hynes HE, et al. A double-blind placebo-controlled trial of granulocyte colony-stimulating factor in elderly patients with previously untreated acute myeloid leukemia: a Southwest oncology group study (9031). Blood. 1998;91:3607-15.

12. Anderson JE, Kopecky KJ, Willman CL, Head D, O'Donnell MR, Luthardt FW, et al. Outcome after induction chemotherapy for older patients with acute myeloid leukemia is not improved with mitoxantrone and etoposide compared to cytarabine and daunorubicin: a Southwest Oncology Group study. Blood. 2002;100: 3869-76

13. Petersdorf SH, Kopecky KJ, Slovak M, Willman C, Nevill T, Brandwein J, et al. A phase 3 study of gemtuzumab ozogamicin during induction and postconsolidation therapy in younger patients with acute myeloid leukemia. Blood. 2013;121:4854-60.

14. Garcia-Manero G, Othus M, Pagel JM, Radich JP, Fang M, Rizzieri DA, et al. SWOG S1203: a randomized phase III study of standard cytarabine plus daunorubicin $(7+3)$ therapy versus idarubicin with high dose cytarabine (IA) with or without vorinostat $(\mathrm{IA}+\mathrm{V})$ in younger patients with previously untreated acute myeloid leukemia (AML) [abstract]. Blood. 2016;128:901.

15. Othus M, Wood BL, Stirewalt DL, Estey EH, Petersdorf SH, Appelbaum FR, et al. Effect of measurable ('minimal') residual disease (MRD) information on prediction of relapse and survival in adult acute myeloid leukemia. Leukemia. 2016;30:2080-3. 